Geometry $8 \mathcal{T}$ opology

Volume 7 (2003) 645-711

Published: 28 October 2003

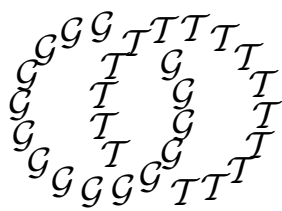

\title{
Calculus III: Taylor Series
}

\author{
Thomas G Goodwillie \\ Department of Mathematics, Brown University \\ Box 1917, Providence RI 02912-0001, USA \\ Email: tomg@math.brown.edu
}

\begin{abstract}
We study functors from spaces to spaces or spectra that preserve weak homotopy equivalences. For each such functor we construct a universal $n$-excisive approximation, which may be thought of as its $n$-excisive part. Homogeneous functors, meaning $n$-excisive functors with trivial $(n-1)$-excisive part, can be classified: they correspond to symmetric functors of $n$ variables that are reduced and 1-excisive in each variable. We discuss some important examples, including the identity functor and Waldhausen's algebraic $K$-theory.
\end{abstract}

\section{AMS Classification numbers Primary: 55P99}

Secondary: $55 \mathrm{U} 99$

Keywords: Homotopy functor, excision, Taylor tower

Proposed: Haynes Miller

Seconded: Ralph Cohen, Gunnar Carlsson
Received: 8 November 2002

Accepted: 20 October 2003

(C) Geometry $\& \mathcal{T}$ Topology Publications 


\section{Introduction}

This paper should have been finished many years ago. It is a continuation of [9] and [10] (which were themselves a little late in coming). The author has no excuse for this procrastination and wishes to apologize to anyone who has been inconvenienced, especially his students and former students.

As in [9] and [10] we are concerned with functors from $\mathcal{C}$, which may be the category of spaces or more generally spaces over a fixed space, to $\mathcal{D}$, which may be either the category of spaces or the category of spectra. It is clear that one could extend the ideas to many other settings. The functors will always be homotopy functors in the sense that they preserve weak homotopy equivalences. We are also concerned with (natural) maps between such functors. We call such a map of functors $F \rightarrow G$ a weak equivalence (or for emphasis an objectwise weak equivalence, or for brevity an equivalence) if for every object $X$ the map $F(X) \rightarrow G(X)$ is a weak equivalence. The goal is to shed light on homotopy functors in general (and on particular ones) by systematically comparing them with homotopy functors of some very special kinds, namely those which satisfy " $n^{\text {th }}$ order excision".

In taking this point of view we are led naturally to speak of the category $\mathcal{F}=\mathcal{F}(\mathcal{C}, \mathcal{D})$ of homotopy functors. Some of our conclusions refer to the homotopy category $h \mathcal{F}$ of homotopy functors, meaning the universal example of a category equipped with a functor from $\mathcal{F}$ that takes every weak equivalence to an isomorphism. For example, some of our results are most neatly expressed as statements to the effect that such and such a functor from one category of functors to another induces an equivalence of homotopy categories. Homotopy categories in this sense can be made by the "generators and relations" construction: a morphism in the homotopy category is an equivalence class of words, where each letter in a word is either a forward arrow (a morphism in $\mathcal{F}$ ) or a backward arrow (a formal inverse to a weak equivalence in $\mathcal{F}$ ) and two words are declared to represent the same morphism if they are related by a sequence of basic moves (composing two forward or two backward arrows, deleting an identity arrow, cancelling a forward arrow and its formal inverse).

The author has recently been reminded that there are set-theoretic objections to bandying this kind of language about too freely, but he does not want to stop doing so. Some ways of resolving this difficulty will be discussed at the end of this introduction.

Of course, if one wants to prove theorems about a homotopy category then one should be prepared to do most of the work in the original category. For example, commutative diagrams in the category of functors will be a ubiquitous 
tool here, but commutative diagrams in the homotopy category are relatively useless.

We now recall the definition of $n^{\text {th }}$ order excision, from section 3 of [10]. Whereas ordinary, or first order, excision has to do with the behavior of a functor on certain square diagrams, $n^{\text {th }}$ order excision has to do with the behavior of a functor on certain cubical diagrams of dimension $n+1$. We briefly recall the definitions. The reader can consult the opening sections of [10] for details, including the specific models we are using for homotopy limits and colimits and the basic definitions and facts about cubical diagrams.

The homotopy functor $F$ is excisive if it takes homotopy pushout squares (also called homotopy cocartesian squares) to homotopy pullback squares (also called homotopy cartesian squares). It is reduced if it takes the final object to an object weakly equivalent to the final object. It is linear if it is both excisive and reduced.

A cubical diagram is called strongly homotopy cocartesian if all of its twodimensional faces are homotopy pushouts. It is called homotopy cartesian if it is a homotopy pullback in the sense that the "first" object is equivalent (by the obvious map) to the homotopy limit of all the others. In this paper we have generally omitted the word "homotopy" in the expressions "(strongly) homotopy (co)cartesian". $F$ is said to be $n$-excisive, or to satisfy $n^{\text {th }}$ order excision, if it takes strongly homotopy cocartesian $(n+1)$-cubical diagrams to homotopy cartesian diagrams. An $n$-excisive functor is always $(n+1)$-excisive.

In [9] we studied the approximation of homotopy functors by excisive functors and codified this in the notion of (first) derivative of a functor. We calculated the derivative, first in some basic examples and then in the example which had given rise to the whole project: stable pseudoisotopy theory (and with it Waldhausen's algebraic $K$-theory functor $A$ ).

In [10] we introduced the class of analytic functors. These are homotopy functors whose deviation from being $n$-excisive is bounded in a certain way for all $n$. Some functors are more analytic than others; an analytic functor is $\rho$-analytic for some integer $\rho$, where a smaller $\rho$ means stronger bounds. We showed that $\rho$-analytic functors behave rather rigidly on the category of $\rho$-connected spaces (and more generally on the category of spaces equipped with $(\rho+1)$ connected maps to a fixed space), in the sense, roughly, that if a map between two such functors induces an equivalence of first derivatives everywhere then its homotopy fiber is constant up to homotopy within the category of $\rho$-connected spaces.

In [5] these results were used to relate $A$ to some functors built out of the free loopspace. 
Here we move from excisive approximation to $n$-excisive approximation, obtaining functors $P_{n} F$ which can be thought of as Taylor polynomials of $F$. We show that if $F$ is $\rho$-analytic and $X$ is $\rho$-connected then as $n$ tends to infinity the $n^{\text {th }}$ approximation converges to $F(X)$ in the strong sense that the connectivity of a certain map $F(X) \rightarrow\left(P_{n} F\right)(X)$ tends to infinity. In other words, the number $\rho$ gives a sort of radius of convergence.

What we were doing in [10] was like showing that a function $f(x)$ is determined, in some region, by $f(0)$ and $f^{\prime}(x)$. Continuing with the same analogy, what we are doing here is like showing that $f(x)$ is determined by $f(0), f^{\prime}(0), f^{\prime \prime}(0)$ and so on.

Here is a sketch of the main results of this paper, presented in a rather different order from the one in which they will be proved.

First consider homotopy functors $\mathcal{T} \stackrel{F}{\longrightarrow} \mathcal{S} p$ from based spaces to spectra. For any such functor, and for any $n \geq 0$, we can make an $n$-excisive functor $\mathcal{T} \stackrel{P_{n} F}{\longrightarrow}$ $\mathcal{S} p$ with a map $F \rightarrow P_{n} F$ that is universal (in a homotopy category) among maps from $F$ to $n$-excisive functors. The functors $\left\{P_{n} F\right\}_{n \geq 0}$ fit together to form a tower, and $F$ maps into the limit of the tower.

If the role of $n^{\text {th }}$ Taylor polynomial is being played by $P_{n} F$, then the role of $n^{\text {th }}$ term in the series is played by the (homotopy) fiber of the map $P_{n} F \rightarrow P_{n-1} F$, which will be denoted by $D_{n} F$. We refer to $P_{n} F$ as the $n^{\text {th }}$ stage of the tower and to $D_{n} F$ as the $n^{\text {th }}$ layer. These homogeneous polynomial functors are the focus of much of the paper.

The constant term $\left(P_{0} F\right)(X)$ is the same, up to natural weak equivalence, as the constant functor $F(*)$.

Up to the same kind of equivalence, the linear (or homogeneous of degree one) functor $\left(D_{1} F\right)(X)$ necessarily has the form $C_{1} \wedge X$ for some fixed spectrum $C_{1}$, at least when restricted to finite complexes $X$. The coefficient spectrum $C_{1}$, which of course is $\left(D_{1} F\right)\left(S^{0}\right)$, is called the derivative of $F$ at the one-point space.

Likewise the homogeneous quadratic functor $\left(D_{2} F\right)(X)$ is necessarily given by

$$
\left(C_{2} \wedge(X \wedge X)\right)_{h \Sigma_{2}}
$$

where $\Sigma_{2}$ is the symmetric group, $C_{2}$ is some spectrum with $\Sigma_{2}$-action, $X \wedge X$ has the obvious action, and the subscript $h \Sigma_{2}$ denotes homotopy orbit spectrum. The coefficient spectrum $C_{2}$, with its $\Sigma_{2}$-action, is called the second derivative of $F$ at the one-point space.

The explanation of why homogeneous quadratic functors always have this form involves symmetric bilinear functors. If $H$ is any 2-excisive functor (still from 
based spaces to spectra), then its second order cross-effect, defined as the total fiber (= homotopy fiber of homotopy fibers) of the square diagram

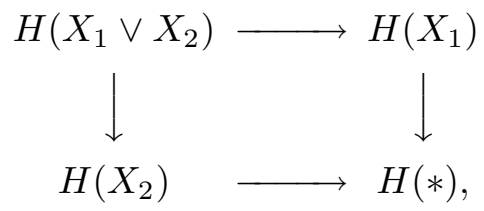

will be a functor $L\left(X_{1}, X_{2}\right)$ of two variables, linear in each variable, and symmetric with respect to interchanging the variables. It turns out that the homogeneous quadratic part of $H$ can be recovered as $L(X, X)_{h \Sigma_{2}}$. A bilinear functor necessarily has the form

$$
L\left(X_{1}, X_{2}\right)=C \wedge\left(X_{1} \wedge X_{2}\right),
$$

and a symmetry on $L$ means a $\Sigma_{2}$-action on $C$.

This pattern persists. For any $n \geq 1$ an $n^{\text {th }}$ degree homogeneous functor $H$ must have the form

$$
H(X)=L(X, \ldots, X)_{h \Sigma_{n}}
$$

where $L$, the $n^{\text {th }}$ order cross-effect of $H$, is a symmetric multilinear functor of $n$ variables. Thus $\left(D_{n} F\right)(X)$ will have the form

$$
\left(C_{n} \wedge(X \wedge \cdots \wedge X)\right)_{h \Sigma_{n}}
$$

at least for finite $X$. The object $C_{n}$, a spectrum with an action of $\Sigma_{n}$, will be called the $n^{\text {th }}$ derivative of $F$ at the one-point space. We also refer to it as the coefficient of the multilinear functor, or of the homogeneous functor.

It is worth emphasizing what brand of stable equivariant homotopy theory is appearing here. Let $G$ be a finite group. To do serious homotopy theory in the category of $G$-spaces one usually takes the weak equivalences to be those equivariant maps which induce weak equivalences on spaces of $H$-fixed points for all subgroups $H$. (For $\mathrm{CW}$ objects this is equivalent to saying that the map has an inverse up to $G$-homotopy.) There is also a very weak notion of equivalence: equivariant maps which are nonequivariantly weak equivalences. Using these as the weak equivalences leads to a theory that is easier and less interesting, but which has its uses. For example, a very weak equivalence of $G$-spaces always induces a weak equivalence of homotopy orbit spaces and also of homotopy fixed point spaces. Every $G$-space is equivalent in the very weak sense to a free $G$-space (product with $E G$ ), and for free $G$-spaces the two kinds of equivalence coincide.

In the course of investigating homogeneous functors we encounter spectra with finite groups acting on them, and this leads us to an obvious stable analogue 
of this easier brand of equivariant homotopy theory: Make a category whose objects are spectra equipped with a $G$-action and whose maps are maps of spectra respecting the action, and call such an equivariant map a (very) weak equivalence if it is nonequivariantly a weak equivalence of spectra. It is these objects (with $G=\Sigma_{n}$ ) up to this kind of equivalence which correspond to homogeneous functors of degree $n$ up to weak natural equivalence.

In order to extend the main ideas of serious equivariant homotopy theory to the stable setting, May and his collaborators created a beautiful and powerful theory of $G$-spectra [12]. We mention this only to say that we do not need it here. From that sophisticated point of view our spectra with $G$-action are what are sometimes called naive $G$-(pre)spectra, namely $G$-spectra involving only trivial actions of $G$ on suspension coordinates. The $G$-spectrum expert will know what else to say about the place of the naive theory in the sophisticated one, but neither the expert nor the novice should have to think about the sophisticated theory to read this paper (unless the expert just cannot help thinking about it).

Returning to the towers, a simple and familiar example is the "Snaith splitting" of the functor $F(X)=\Sigma^{\infty} \Omega \Sigma X$. Its $n^{\text {th }}$ homogeneous part is $\left(D_{n} F\right)(X) \sim$ $\Sigma^{\infty}\left(X^{\wedge n}\right)$, and its tower splits: $P_{n} F \sim \prod_{1 \leq k \leq n} D_{k} F$. The limit of the tower is the product of all the layers. If $X$ is connected then the tower converges to $F(X)$ in the sense that the map from $F(X)$ to the homotopy limit of the tower is a weak equivalence, and in fact in the stronger sense that the map from $F(X)$ to $\left(P_{n} F\right)(X)$ has a connectivity tending to infinity with $n$.

A related example is $F(X)=\Sigma^{\infty} \Omega X$. Here we have

$$
\left(D_{n} F\right)(X) \sim \Omega^{n} \Sigma^{\infty}\left(X^{\wedge n}\right) .
$$

(We will give a quick proof of this using the previous example and the general fact $P_{n}(F \circ \Sigma) \sim\left(P_{n} F\right) \circ \Sigma$. See Example 1.20 below. $)$ The $n^{\text {th }}$ derivative is the wedge of $n$ factorial copies of the spectrum $S^{-n}$ permuted transitively by $\Sigma_{n}$. The tower does not split. It converges (in the strong sense again) when the space $X$ is 1 -connected.

A more general example is $F(X)=\Sigma^{\infty} \operatorname{Map}_{*}(K, X)$ where $K$ is a based finite complex and $M a p_{*}$ means the space of based maps. Here the $n^{\text {th }}$ derivative is the S-dual of a based complex with $\Sigma_{n}$-action, namely the quotient of the smash product $K^{\wedge n}$ by the fat diagonal. $F$ is $\operatorname{dim}(K)$-analytic, and the tower converges to $F(X)$ if $X$ is $\operatorname{dim}(K)$-connected. Arone [1] thoroughly explored this class of examples, giving a concrete description of the stages $P_{n} F$ and not just the layers $D_{n} F$.

The $n^{\text {th }}$ coefficient of Waldhausen's $A(X)$ is again the S-dual of a based finite complex with $\Sigma_{n}$-action, namely $\left(\Sigma_{n}\right)_{+} \wedge_{C_{n}} S^{n-1}$, where $C_{n} \subset \Sigma_{n}$ is the 
transitive cyclic subgroup of order $n$ and the sphere $S^{n-1}$ is the one-point compactification of the reduced regular representation of $C_{n}$.

So far we have been concerned with functors from based spaces to spectra. We now discuss three different variants of this setup: functors from unbased spaces, functors from spaces over a fixed space, and functors to spaces. In these new settings the Taylor tower construction goes through with no change, but some additional work is needed to understand what a homogeneous functor looks like.

The switch from functors $\mathcal{T} \rightarrow \mathcal{S} p$ of based spaces to functors $\mathcal{U} \rightarrow \mathcal{S} p$ of unbased spaces is fairly innocuous. If $\mathcal{T} \stackrel{\phi}{\rightarrow} \mathcal{U}$ is the forgetful functor then $\left(P_{n} F\right) \circ \phi=P_{n}(F \circ \phi)$ and $\left(D_{n} F\right) \circ \phi=D_{n}(F \circ \phi)$.

A snag appears when one tries to relate homogeneous functors to symmetric multilinear functors, since the definition of the cross-effect requires basepoints. The good news is that in the end this does not matter: homogeneous functors $\mathcal{T} \rightarrow \mathcal{S} p$ of any degree extend uniquely (in an appropriate up-to-natural-weakequivalence sense) to $\mathcal{U}$, and the same is true for symmetric multilinear functors in any number of variables. The proof involves the left adjoint $\psi$ of $\phi$, in other words the functor that adds a disjoint basepoint to an unbased space. For example, although the usual definition of the suspension spectrum of a space $X$ requires $X$ to be based, there is a well-known extension to unbased spaces. It associates to $X$ the homotopy fiber of

$$
\Sigma^{\infty} \psi(X) \rightarrow \Sigma^{\infty} \psi(*) .
$$

If $X$ is based then this is naturally (with respect to based maps) equivalent to $\Sigma^{\infty} X$, and on the other hand if $\mathcal{U} \stackrel{L}{\longrightarrow} \mathcal{S} p$ is any linear functor such that $L \circ \phi$ is (naturally equivalent to) $\Sigma^{\infty}$ then $L$ must be as defined above. This was explained in [9] and is generalized to higher degrees here.

Note that for inhomogeneous functors this goes very wrong. For example, the 1-excisive functor

$$
\Sigma^{\infty} \psi(*) \vee \text { hofiber }\left(\Sigma^{\infty} \psi(X) \rightarrow \Sigma^{\infty} \psi(*)\right) .
$$

from $\mathcal{U}$ to $\mathcal{S} p$ is genuinely different from $\Sigma^{\infty} \psi(X)$, but the difference disappears after restriction to $\mathcal{T}$. (The empty set is the only space $X$ at which the functors disagree, but even for nonempty spaces the equivalence cannot be chosen to be natural with respect to unbased maps.) An example of a 2-excisive functor $\mathcal{T} \rightarrow \mathcal{S} p$ which does not extend to $\mathcal{U}$ at all is the suspension spectrum of

$$
F(X)=\left((X \wedge X) / \Delta_{X}\right)_{\Sigma_{2}}
$$


the orbit space for the $\Sigma_{2}$-action on the quotient of $X \wedge X$ by the diagonal. If $X$ is the disjoint union of a point and a circle then the rational homology of $F(X)$ depends on where the basepoint is placed in $X$.

The second switch, from functors of spaces to functors of spaces over a fixed space, is something like the switch from MacLaurin series to general Taylor series. Instead of building, for each space $X$, a tower that has $F(*)$ at the bottom and attempts to converge to $F(X)$, one can build, for each map of spaces $X \rightarrow Y$, a tower that has $F(Y)$ at the bottom and attempts to converge to $F(X)$. If we fix $Y$ and think of everything in sight as a functor of spaces over $Y$ then the $n^{t h}$ stage of the tower is $n$-excisive. As in the case $Y=*$, there are two options, each with its own technical advantages: the category $\mathcal{U}_{Y}$ of plain spaces over $Y$ and the category $\mathcal{T}_{Y}$ of spaces over $Y$ equipped with a section. We sometimes refer to the latter as fiberwise based spaces over $Y$.

The correspondence between homogeneous and symmetric multilinear functors works the same for spaces over $Y$ as it does for spaces, but the business of describing a multilinear functor by coefficient spectra becomes more complicated when $Y$ has more than one point. As a trivial example, to describe a linear functor of spaces over the two-point space $\left\{y_{1}, y_{2}\right\}$ one needs two spectra. In general a linear functor $L$ of spaces over $Y$ determines a spectrum for each point $y \in Y$, namely the coefficient $L\left(Y \vee_{y} S^{0}\right)$ of the linear functor $Z \mapsto L\left(Y \vee_{y} Z\right)$ from $\mathcal{T}$ to $\mathcal{S} p$, where $Y \vee_{y} Z$ means the wedge sum of $(Y, y)$ with the based space $Z$, viewed as a space over $Y$. This spectrum depends "continuously" on the point $y$ in some sense. If $Y$ is path-connected then up to weak homotopy equivalence the spectrum is independent of the point; but there is a twisting that must not be overlooked.

In [9] we defined the differential $D_{Y} F$ of a functor $\mathcal{U} \stackrel{F}{\longrightarrow} \mathcal{S} p$ at a space $Y$ to be a linear functor from spaces over $Y$ to spectra, the linear approximation to the functor

$$
(X \rightarrow Y) \mapsto \operatorname{hofiber}(F(X) \rightarrow F(Y))
$$

We also defined the derivative to be a spectrum $\partial_{y} F(Y)$ depending on a space $Y$ and a point $y \in Y$, namely $\left(D_{Y} F\right)\left(Y \vee_{y} S^{0}\right)$; it might be called the $y$ coefficient of $D_{Y} F$ or the partial derivative of $F$ at $Y$ in the $y$ direction. The relationship between the differential $D_{Y} F$ (a linear functor of spaces over $Y$ ) and the derivative $\partial_{y} F(Y)$ (a spectrum) is something like the relationship between the differential of a function (a linear function on the tangent space) and a partial or directional derivative (a number, which specifies the behavior of that linear function on a certain one-dimensional tangent subspace). The spectrum $\partial_{y} F(Y)$ records the derivative of $F$ at $Y$ in the "direction" given by $y$. Here we make the multilinear generalization, defining the $n^{\text {th }}$ differential 
to be the symmetric multilinear functor corresponding to the $n^{\text {th }}$ layer of the Taylor tower and defining the $n^{\text {th }}$ derivative

$$
\partial_{y_{1}, \ldots, y_{n}}^{(n)} F(Y)
$$

to be its value at $\left(Y \vee_{y_{1}} S^{0}, \ldots, Y \vee_{y_{n}} S^{0}\right)$.

The third switch, from spectrum-valued to space-valued functors, brings a real surprise. The construction of the Taylor series goes through with no change. If the functor $F$ happens to be $\Omega^{\infty} G$ for some spectrum-valued functor $G$, then we have $P_{n} F=\Omega^{\infty} P_{n} G$ and $D_{n} F=\Omega^{\infty} D_{n} G$. The surprise is that although most functors are not of the form $\Omega^{\infty} G$ this is not reflected at the homogeneous level: every homogeneous functor $\mathcal{T} \rightarrow \mathcal{T}$ of degree $n \geq 1$ is infinitely deloopable, in fact in a unique and functorial way. Thus $D_{n} F$ always has the form $\Omega^{\infty}\left(\left(C_{n} \wedge X^{\wedge n}\right)_{h \Sigma_{n}}\right)$ and even in the space-valued case we can introduce a spectrum called the $n^{\text {th }}$ derivative of the functor, or the coefficient spectrum of the $n^{\text {th }}$ homogeneous layer.

A central example of a functor $\mathcal{T} \rightarrow \mathcal{T}$ that is interesting from this point of view is the identity. Let us call it $I$. Its first derivative is the sphere spectrum. It is easy to see, in any of a number of ways, that the $n^{\text {th }}$ derivative is equivalent to the wedge sum of $(n-1)$ ! copies of the $(1-n)$-sphere spectrum, with $\Sigma_{n}$ acting in such a way that the subgroup $\Sigma_{n-1}$ freely permutes the summands. To identify $D_{n} I$ one has to know the action of the full group $\Sigma_{n}$. Johnson [11] gave an explicit finite complex with $\Sigma_{n}$-action whose S-dual is the answer. Arone and Mahowald [4] gave a different answer of that kind, showed that it was equivalent to Johnson's, and used it to make some very interesting calculations.

A basic example of an inhomogeneous functor that does not deloop is $P_{2} I$, the 2 -excisive approximation of the identity functor. The functor $P_{1} I$ is of course $Q=\Omega^{\infty} \Sigma^{\infty} .\left(P_{2} I\right)(X)$ is another standard object, the homotopy fiber of the James-Hopf map

$$
Q X \rightarrow Q\left((X \wedge X)_{h \Sigma_{2}}\right) .
$$

(which is not induced by a spectrum map from $\Sigma^{\infty} X$ to $\Sigma^{\infty}(X \wedge X)_{h \Sigma_{2}}$ ).

One notable feature of this last example turns out to be quite general: the fibration sequence

$$
D_{n} F \rightarrow P_{n} F \rightarrow P_{n-1} F
$$

can always be extended one step to the right, as long as the constant functor $P_{0} F$ is contractible. This falls out of the proof of (and is essentially equivalent to) the deloopability of homogeneous functors.

The construction of the Taylor tower also goes through for functors taking values in unbased instead of based spaces, but in that case it is nonsense to 
speak of the layers $D_{n} F$ as functors, since that would involve using "the" fiber of a map of unbased spaces. This small fussy point is really not so small. The identity functor $I$ above was from based spaces to based spaces. Let $J$ be the identity functor from unbased spaces to unbased spaces. Then $\left(P_{1} J\right)(X)$ is the homotopy fiber of

$$
Q\left(X_{+}\right) \rightarrow Q S^{0},
$$

but not with respect to the usual basepoint in $Q S^{0}$. In particular $\left(P_{1} J\right)(\emptyset)$ is empty, so that there is no natural basepoint in $\left(P_{1} J\right)(X)$. This seriously interferes with defining $D_{2} J . P_{1} J$ is excisive and reduced, but perhaps too badly twisted to be rightly called linear. For such reasons we hesitate to even speak of homogeneous functors to unbased spaces.

The paper is organized as follows:

Section 1 defines the tower $\left\{P_{n} F\right\}$ in the general case, proves that $P_{n} F$ is $n$-excisive, establishes the universal mapping property of $P_{n} F$, and notes the convergence of the tower in the case of an analytic functor $F$.

Section 2 shows that homogeneous space-valued functors can be functorially delooped and concludes that they correspond precisely to homogeneous spectrumvalued functors.

Section 3 establishes the correspondence between homogeneous functors and symmetric multilinear functors in the case of functors from fiberwise based spaces over $Y$ to spectra. By Section 2 this result extends to space-valued functors.

Section 4 shows that for homogeneous functors, and also for symmetric multilinear functors, it does not matter whether the domain category is (fiberwise) based or unbased spaces, so that some of the results of Section 3 extend to functors of plain spaces over $Y$.

Section 5 establishes notation for the symmetric multilinear functors that (according to sections 2-4) encode the homogeneous layers of a Taylor tower, and develops the idea of coefficient spectra for multilinear functors.

Section 6 establishes a useful tool for working out examples. The key point is that the $n^{\text {th }}$ cross effect of the homogeneous functor $D_{n} F$ can be made by "multilinearizing" the $n^{\text {th }}$ cross effect of $F$ itself.

Section 7 works out the $n^{\text {th }}$ derivatives of functors like $\Sigma^{\infty} M a p_{*}(K,-)$.

Section 8 recalls and discusses known results on the Taylor tower of the identity.

Section 9 indicates how to get the $n^{\text {th }}$ derivative of Waldhausen's $A$, taking the first derivative as starting point. 
A few words about set theory are in order. We all know that we must not speak of the set of all sets or the set of all topological spaces; set theory, as formulated to avoid Russell-type paradoxes, does not allow it. And since the category $\mathcal{C}$ of spaces is not small, there may be objections to speaking of the category of all functors, or homotopy functors, from $\mathcal{C}$ to $\mathcal{D}$ in that case. Even if $\mathcal{C}$ and $\mathcal{D}$ are both small, the functor category will not be small in general, so that it is illegal to make a new category by using generators and relations to invert some morphisms.

We choose to dodge this as follows. As usual when basing mathematics on set theory, we begin by fixing a universe $\mathcal{U}$ of sets. Now by topological spaces we mean those whose point sets are sets in this strict sense. The category of such spaces is not a small category, any more than the set of all $(\mathcal{U}$-)sets is a $(\mathcal{U}$-)set. Nevertheless, if we are willing to work in two universes $[14 ;$ I.6], we are not in such bad shape. Introduce a larger universe $\mathcal{U}^{\prime}$ in which the set of all $\mathcal{U}$-sets is a set. The category of all $\left(\mathcal{U}\right.$-)spaces is then $\mathcal{U}^{\prime}$-small. In $\mathcal{U}^{\prime}$ there can be no objection to speaking of the category of all functors from spaces to spaces, or of the full subcategory of homotopy functors. To invert the weak equivalences by generations and relations, one can always pass to a third universe in which the category of homotopy functors is small.

This solution will not suit all tastes. It may be a bit wasteful and it may be a bit crude. For some more refined purposes it will be inadequate. For example, if one wants to introduce some sort of space of maps between two homotopy functors such that the set of components will be the set of morphisms in the homotopy category, then it will certainly be a drawback to find that this "function space" is not a space in the original sense. In general, if one wants to work very seriously with the homotopy category of functors then one will probably want to introduce a closed model structure on the functor category, with all the benefits that that brings. (In fact, although we have not attempted to do so, there are certainly many reasons for reworking this whole theory in the context of closed model categories. The objects of study should be functors from one (small?) model category to another, subject to some mild axioms, and the category of homotopy functors should turn out to be a model category, too.)

On the other hand, in this paper the role of the homotopy categories is a modest one: they are used here mainly as shorthand, to make some sentences briefer and more readily comprehensible than they otherwise would be. The "multiple universes" device allows us to use this shorthand without telling any lies, but without making the catogory of spaces artificially small. 


\section{The Taylor tower}

Let $\mathcal{C} \stackrel{F}{\longrightarrow} \mathcal{D}$ be a homotopy functor, where $\mathcal{C}=\mathcal{C}_{Y}$ is either $\mathcal{U}_{Y}$ or $\mathcal{T}_{Y}$ and $\mathcal{D}$ is either $\mathcal{U}, \mathcal{T}$, or $\mathcal{S} p$. Let $n \geq 0$.

The $n$-excisive approximation $P_{n} F$ will be constructed by the infinite iteration of another construction $T_{n}$ which is designed to bring the functor $F$ a little closer to being $n$-excisive. The special case when $n=1$ was treated in [9; Def. 1.10], where $T_{1}$ and $P_{1}$ were called $T$ and $P$. Thus if $Y=*$ and $F(*)=*$ then $T_{1} F \sim \Omega F \Sigma$ and $P_{n} F \sim$ hocolim $_{i \geq 0} \Omega^{i} F \Sigma^{i}$.

The definition of $T_{n}$ uses the fiberwise join over $Y$, as introduced in the proof of $[10 ; 5.1]$. Let $X$ be a space over $Y$ and let $U$ be a space. (In most applications $U$ will be a discrete finite set.) The fiberwise join is the space

$$
X *_{Y} U=\operatorname{hocolim}(X \leftarrow X \times U \rightarrow Y \times U)
$$

considered as a space over $Y$. The name signifies that the functor "join with $U$ " is being applied to all the fibers of $X \rightarrow Y$.

If $U$ has one element, then $X *_{Y} U \rightarrow Y$ is the fiberwise cone of $X$ over $Y$ (the mapping cylinder of $X \rightarrow Y$ considered as a space over $Y$ ). If $U$ has two elements then $X *_{Y} U \rightarrow Y$ is the fiberwise suspension and will sometimes be denoted by $\Sigma_{Y} X$.

It should be noted that this construction has the best of both worlds, in that on the one hand each fiber of $X *_{Y} U \rightarrow Y$ is homeomorphic to the join with $U$ of the corresponding fiber of $X \rightarrow Y$, and on the other hand each homotopy fiber is weakly homotopy equivalent to the join with $U$ of the corresponding homotopy fiber of $X \rightarrow Y$.

Recall that even if $\mathcal{C}$ is $\mathcal{T}_{Y}$ rather than $\mathcal{U}_{Y}$ then $X *_{Y} U$ is still in $\mathcal{C}$; it inherits a section from $X$. In other words, if $X$ is a fiberwise based space over $Y$ then $X *_{Y} U$ is also canonically fiberwise based, without for example choosing a point in $U$.

The object $X *_{Y} U$ depends (bi-)functorially on $X$ and $U$. Note also that there is a natural isomorphism of spaces over $Y$ :

$$
\left(X *_{Y} U\right) *_{Y} V \cong X *_{Y}(U * V)
$$

where $U * V$ is the ordinary join of two spaces.

Let $\mathcal{P}(\underline{n+1})$ be the poset of subsets of $\underline{n+1}=\{1, \ldots, n+1\}$. Any object $X \in \mathcal{C}$ yields an $(n+1)$-dimensional cubical diagram in $\mathcal{C}$.

$$
\begin{gathered}
\mathcal{P}(\underline{n+1}) \rightarrow \mathcal{C} \\
U \mapsto X *_{Y} U
\end{gathered}
$$


Consider the composed functor $U \mapsto F\left(X *_{Y} U\right)$, a cubical diagram in $\mathcal{D}$. The homotopy limit of its restriction to $\mathcal{P}_{0}(\underline{n+1})$, the poset of nonempty subsets of $\underline{n+1}$, will be called $\left(T_{n} F\right)(X)$. Clearly this yields a homotopy functor $\mathcal{C} \stackrel{\overline{T_{n} F}}{\longrightarrow} \mathcal{D}$. There is a natural map $F \stackrel{t_{n} F}{\longrightarrow} T_{n} F$, since any cubical diagram determines a map from the "initial" object to the homotopy limit of the others (see [10; Def. 1.2]):

$$
F(X)=F\left(X *_{Y} \emptyset\right) \stackrel{t_{n} F}{\longrightarrow} \operatorname{holim}_{U \in \mathcal{P}_{0}(\underline{n+1})} F\left(X *_{Y} U\right)=\left(T_{n} F\right)(X)
$$

Let $\left(P_{n} F\right)(X)$ be the sequential homotopy colimit of the diagram

$$
F(X) \stackrel{\left(t_{n} F\right)(X)}{\longrightarrow}\left(T_{n} F\right)(X) \stackrel{\left(t_{n} T_{n} F\right)(X)}{\longrightarrow}\left(T_{n}^{2} F\right)(X) \stackrel{\left(t_{n} T_{n}^{2} F\right)(X)}{\longrightarrow} \ldots
$$

Clearly $P_{n} F$ is a homotopy functor and we have a natural map $F \stackrel{p_{n} F}{\longrightarrow} P_{n} F$.

The cubical diagram $U \mapsto X *_{Y} U$ is strongly cocartesian for all $X$. Therefore if the functor $F$ is $n$-excisive then the maps $t_{n} F$ and $p_{n} F$ will be weak equivalences for all $X$. In this sense $n$-excisive functors are unchanged by $T_{n}$ and $P_{n}$.

We will see below that $P_{n} F$ is always $n$-excisive, and that (in a homotopy category) it is the best $n$-excisive approximation to $F$ in a categorical sense.

1.1 Remark If the map $X \rightarrow Y$ is $m$-connected, then for nonempty $U$ the map $X *_{Y} U \rightarrow Y$ is $(m+1)$-connected, and therefore $\left(T_{n}^{i} F\right)(X)$ depends only on the behavior of $F$ on objects whose structural maps to $Y$ are $(m+i)$ connected. In this sense the Taylor approximations of a functor $\mathcal{C}_{Y} \stackrel{F}{\longrightarrow} \mathcal{D}$ depend only on the restriction of the functor to objects "arbitrarily close to $Y$ " (just as the Taylor expansion of $f(x)$ in powers of $x-y$ depends only on the behavior of $f(x)$ in an arbitrarily small neighborhood of $y$ ). A related comment is that $P_{n}$ commutes (up to natural isomorphism) with fiberwise suspension:

$$
P_{n}\left(F \circ \Sigma_{Y}\right) \cong\left(P_{n} F\right) \circ \Sigma_{Y} .
$$

This follows from the natural isomorphism

$$
\Sigma_{Y}\left(X *_{Y} U\right) \cong\left(\Sigma_{Y} X\right) *_{Y} U .
$$

Before giving the general proof that $P_{n} F$ is $n$-excisive, we give a different proof under the added assumption that $F$ is stably $n$-excisive $[10 ; 4.1]$, and in this case we show that $P_{n} F$ approximates $F$ not only in a categorical sense but also from the point of view of connectivity.

The first task is to show that if $F$ is stably $n$-excisive then $T_{n} F$ is more nearly $n$-excisive than $F$ is, and that $T_{n} F$ agrees with $F$ to $n^{\text {th }}$ order in the following sense: 
1.2 Definition A map $u: F \rightarrow G$ between two functors from $\mathcal{C}_{Y}$ to $\mathcal{D}$ is said to satisfy $O_{n}(c, \kappa)$ if, for every $k \geq \kappa$, for every object $X$ of $\mathcal{C}_{Y}$ such that the map $X \rightarrow Y$ to the final object is $k$-connected, the map $u_{X}: F(X) \rightarrow G(X)$ is $(-c+(n+1) k)$-connected. We say that $F$ and $G$ agree to order $n$ (via the map $u$ ) if this holds for some constants $c$ and $\kappa$.

1.3 Remark The letter $O$ stands for "osculation". This condition on (a map between) two functors is analogous to a condition on two functions (say real functions of one or several variables). The functions $f$ and $g$ can be said to agree to $n^{\text {th }}$ order at $y$ if there are constants $C$ and $K$ such that for every $x$ such that $|x-y| \leq K$ we have $|f(x)-g(x)| \leq C|x-y|^{n+1}$.

The condition $E_{n}(c, \kappa)$, stable $n^{\text {th }}$ order excision, was defined in [10: Def. 4.1].

1.4 Proposition If $F$ satisfies $E_{n}(c, \kappa)$, then

(1) $T_{n} F$ satisfies $E_{n}(c-1, \kappa-1)$ and

(2) $t_{n} F: F \rightarrow T_{n} F$ satisfies $O_{n}(c, \kappa)$.

Proof The second conclusion is immediate from the definitions. For the first, note that the functor $X \mapsto X *_{Y} U$ from $\mathcal{C}$ to $\mathcal{C}$ preserves strongly cocartesian cubes and (unless $U$ is empty) increases the connectivity of maps. It follows immediately that for each nonempty $U$ the functor $X \mapsto F\left(X *_{Y} U\right)$ from $\mathcal{C}$ to $\mathcal{D}$ satisfies $E_{n}(c-(n+1), \kappa-1)$. By [10; 1.20], $T_{n} F$ satisfies $E_{n}(c-1, \kappa-1)$.

1.5 Proposition If $F$ is stably $n$-excisive, then

(1) $P_{n} F$ is $n$-excisive and

(2) $F$ agrees with $P_{n} F$ to order $n$ (via $p_{n} F$ ).

Proof Suppose that $F$ satisfies $E_{n}(c, \kappa)$. By 1.4(1) and induction, $T_{n}^{i} F$ satisfies $E_{n}(c-i, \kappa-i)$. This easily implies that $P_{n} F$ is $n$-excisive. We also find, by 1.4(2), that $t_{n} T_{n}^{i} F$ satisfies $O_{n}(c-i, \kappa-i)$, and in particular $O_{n}(c, \kappa)$, for all $i$. It follows easily that $O_{n}(c, \kappa)$ is also satisfied by the composed maps $\left(t_{n} T_{n}^{i} F\right) \circ \ldots \circ t_{n} F$ and in the limit by $p_{n} F$.

1.6 Proposition Let $F \stackrel{u}{\rightarrow} G$ be a map between homotopy functors. If $F$ and $G$ agree to $n^{\text {th }}$ order via $u$, then the induced map $P_{n} F \stackrel{P_{n} u}{\longrightarrow} P_{n} G$ is an equivalence. The converse holds if $F$ and $G$ are stably $n$-excisive. 
Proof Suppose $u$ satisfies $O_{n}(c, \kappa)$. For each nonempty finite set $U$ the resulting map of functors

$$
F\left(-*_{Y} U\right) \rightarrow G\left(-*_{Y} U\right)
$$

satisfies $O_{n}(c-(n+1), \kappa-1)$; again we have used the fact that the functor $X \mapsto X *_{Y} U$ from $\mathcal{C}$ to $\mathcal{C}$ preserves strongly cocartesian cubical diagrams and increases the connectivity of maps. Therefore, using $[10 ; 1.20]$ as in the proof of 1.4, $T_{n} F \stackrel{T_{n} u}{\longrightarrow} T_{n} G$ satisfies $O_{n}(c-1, \kappa-1)$. By induction, $T_{n}^{i} F \stackrel{T_{n}^{i} u}{\longrightarrow} T_{n}^{i} G$ satisfies $O_{n}(c-i, \kappa-i)$. It follows, letting $i$ tend to infinity, that $P_{n} F \rightarrow P_{n} G$ is an equivalence. For the converse, use 1.5(2) and the commutative diagram

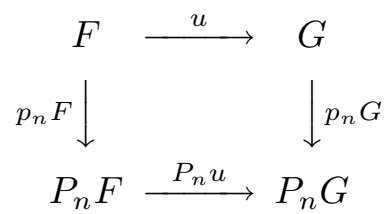

Note that when $F$ is stably $n$-excisive $P_{n} F$ can be characterized, up to natural equivalence, as the only $n$-excisive functor that agrees to $n^{\text {th }}$ order with $F$.

The remaining results of Section 1 (except for the last sentence of 1.13) have nothing to do with connectivity. In particular, functors are not assumed to satisfy any kind of stable excision hypothesis.

1.7 Proposition Up to equivalence,

(1) $T_{n}$ commutes with holim

(2) $P_{n}$ commutes with finite holim

(3) $T_{n}$ and $P_{n}$ commute with hofiber.

(4) $T_{n}$ and $P_{n}$ commute with filtered (in particuloar sequential) hocolim.

(5) for spectrum-valued functors, both $T_{n}$ and $P_{n}$ commute with any hocolim.

Proof and explanation The main point is that holim commutes with holim, hocolim commutes with hocolim, and, up to equivalence, finite holim commutes with filtered hocolim.

(1) This one is true up to isomorphism: If $\left\{F_{\alpha}\right\}$ is any sort of diagram of homotopy functors $\mathcal{C} \stackrel{F_{\alpha}}{\longrightarrow} \mathcal{D}$ and $F$ is given by $F(X)=\operatorname{holim}_{\alpha} F_{\alpha}(X)$ then we have

$$
\begin{aligned}
\left(T_{n} F\right)(X) & \cong \underset{U}{\operatorname{holim}} \operatorname{holim}_{\alpha} F_{\alpha}\left(X *_{Y} U\right) \\
& \cong \operatorname{holim}_{\alpha} \operatorname{holim}_{U} F_{\alpha}\left(X *_{Y} U\right) \\
& \cong \operatorname{holim}_{\alpha}\left(T_{n} F_{\alpha}\right)(X)
\end{aligned}
$$

Geometry $8 \mathcal{G}$ Topology, Volume 7 (2003) 
(2) In the same situation we have a natural map

$$
\begin{aligned}
\left(P_{n} F\right)(X) & \cong \underset{i}{\operatorname{hocolim}}\left(T_{n}^{i} F\right)(X) \\
& \cong \underset{i}{\operatorname{hocolim}} \operatorname{holim}\left(T_{n}^{i} F_{\alpha}\right)(X) \\
& \rightarrow \underset{\alpha}{\operatorname{holim}} \operatorname{hocolim}\left(T_{n}^{i} F_{\alpha}\right)(X) \\
& \cong \underset{\alpha}{\operatorname{holim}}\left(P_{n} F_{\alpha}(X)\right.
\end{aligned}
$$

It is an equivalence in the case when $\left\{F_{\alpha}\right\}$ is a finite diagram.

(3) This follows from (1) and (2). We have

$$
\begin{aligned}
P_{n} \operatorname{hofiber}(F \rightarrow G) & \cong P_{n} \operatorname{holim}(F \rightarrow G \leftarrow *) \\
& \sim \operatorname{holim}\left(P_{n} F \rightarrow P_{n} G \leftarrow P_{n} *\right) \\
& \sim \operatorname{holim}\left(P_{n} F \rightarrow P_{n} G \leftarrow *\right) \\
& \cong \operatorname{hofiber}\left(P_{n} F \rightarrow P_{n} G\right)
\end{aligned}
$$

and likewise for $T_{n}$.

(4) $T_{n}$ is a finite holim and $P_{n}$ is a hocolim of finite holim's.

(5) Since hocolims commute, hocolim preserves cocartesian cubes. Thus hocolim of spectra preserves cartesian cubes. This implies (using $[10 ; 1.19]$ ) that $T_{n}$ commutes with hocolim up to equivalence. Therefore the same holds for $P_{n}=$ $\operatorname{hocolim}_{i} T^{i}$.

Let $\mathcal{F}(\mathcal{C}, \mathcal{D})$ be the category whose objects are the homotopy functors from $\mathcal{C}$ to $\mathcal{D}$, and whose morphisms are the natural maps. Let $h \mathcal{F}(\mathcal{C}, \mathcal{D})$ be its homotopy category; it has the same objects and is obtained by formally inverting the equivalences. Because the functors $T_{n}$ and $P_{n}$ from $\mathcal{F}(\mathcal{C}, \mathcal{D})$ to itself take equivalences to equivalences, they give rise to functors from the homotopy category to itself. Morphisms in $h \mathcal{F}(\mathcal{C}, \mathcal{D})$ are called weak maps. Any functor weakly isomorphic to an $n$-excisive functor is $n$-excisive.

1.8 Theorem For any homotopy functor $\mathcal{C} \stackrel{F}{\longrightarrow} \mathcal{D}$, the functor $P_{n} F$ is $n$ excisive. In the homotopy category $h \mathcal{F}(\mathcal{C}, \mathcal{D}), p_{n} F$ is the universal map from $F$ to an $n$-excisive functor.

The key to 1.8 is the following:

1.9 Lemma Let $\mathcal{X}$ be any strongly cocartesian $(\underline{n+1})$-cube in $\mathcal{C}$, and let $F$ be any homotopy functor. Then the map of cubes

$$
F(\mathcal{X}) \stackrel{\left(t_{n} F\right)(\mathcal{X})}{\longrightarrow}\left(T_{n} F\right)(\mathcal{X})
$$

factors through some cartesian cube. 
Proof of 1.8, assuming 1.9 Let $\mathcal{X}$ be any strongly cocartesian $(\underline{n+1})$-cube in $\mathcal{C}$, and consider the diagram of cubes

$$
F(\mathcal{X}) \rightarrow\left(T_{n} F\right)(\mathcal{X}) \rightarrow\left(T_{n}^{2} F\right)(\mathcal{X}) \rightarrow \ldots
$$

which leads by hocolim to the cube $\left(P_{n} F\right)(\mathcal{X})$. By 1.9 each of the maps of cubes displayed above factors through some cartesian cube. Therefore the cube $\left(P_{n} F\right)(\mathcal{X})$ is equivalent to a sequential hocolim of cartesian cubes, and so it is itself cartesian. This shows that $P_{n} F$ is $n$-excisive.

For the existence half of the universal mapping property, let $F \stackrel{u}{\rightarrow} P$ be any weak map to an $n$-excisive functor. The diagram

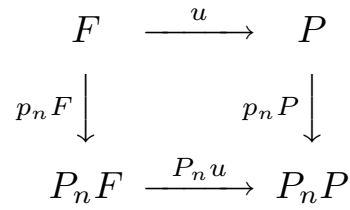

shows that $u$ factors through $p_{n} F$, since $p_{n} P$ as a weak map is invertible.

For the uniqueness we must show that if $P$ is $n$-excisive then a weak map $P_{n} F \stackrel{v}{\rightarrow} P$ is determined by the composition $v \circ p_{n} F$. It suffices if in the diagram of weak maps

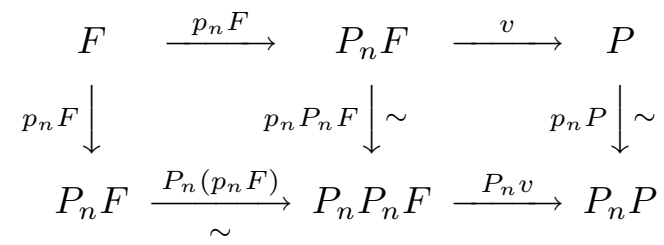

the marked $(\sim)$ arrows are invertible, for then $v$ is determined by $P_{n} v$, which is determined by $P_{n} v \circ P_{n}\left(p_{n} F\right)=P_{n}\left(v \circ p_{n} F\right)$, which is of course determined by $v \circ p_{n} F$.

The marked vertical arrows are equivalences because $P_{n} F$ and $P$ are $n$-excisive. In order for $P_{n}\left(p_{n} F\right)$ to be an equivalence, it will be enough (by 1.7(4)) if $P_{n}\left(t_{n} F\right)$ is an equivalence. Define the functor $J_{U} F$ by $\left(J_{U} F\right)(X)=F\left(X *_{Y} U\right)$. In the diagram

$$
\begin{aligned}
P_{n} F \stackrel{P_{n}\left(t_{n} F\right)}{\longrightarrow} P_{n} T_{n} F \cong P_{n}\left(\operatorname{holim}_{U \in \mathcal{P}_{0}(\underline{n+1})} J_{U} F\right) \\
\stackrel{\sim}{\longrightarrow} \operatorname{holim}_{U \in \mathcal{P}_{0}(\underline{n+1)})} P_{n} J_{U} F \cong \operatorname{holim}_{U \in \mathcal{P}_{0}(\underline{n+1})} J_{U} P_{n} F
\end{aligned}
$$

the second arrow is an equivalence by 1.7(2). The composition is also an equivalence; this simply means that for every $X$ the functor $P_{n} F$ takes the cube $\left\{X *_{Y} U\right\}$ to a cartesian cube, and it is true because $P_{n} F$ is $n$-excisive. It follows that $P_{n}\left(t_{n} F\right)$ is an equivalence. 
The fact that $P_{n}\left(p_{n} F\right)=p_{n} P_{n} F$ in the homotopy category follows from 1.8; if we had had it before 1.8, then we could have skipped the last part of the proof of 1.8 .

1.10 Remark Theorem 1.8 is one of several statements in this paper for which we have two different proofs: an older one which requires some kind of stable excision hypothesis, and a newer one which has nothing to do with connectivity. Another such statement is Theorem 2.1, which also depends (near the end of the proof of 2.2) on 1.9. Another is 3.1, and another is 6.1. The older proofs have the advantage of a certain common-sense quality, and if what we really care about here is convergent Taylor towers then the older proofs are good enough; but the fact that the theorems are still true without connectivity hypotheses is striking and the newer proofs are perhaps worth looking at, too.

Proof of 1.9 (Any reader who, like the author, finds this proof a little opaque, may wish to look at it again after reading the proof of 3.2, which is related but simpler.) Let us write $n$ instead of $n+1$. For subsets $T, U_{1}, \ldots, U_{n}$ of $\underline{n}$, define $\hat{\mathcal{X}}\left(T, U_{1}, \ldots, U_{n}\right)$ to be

$$
\operatorname{hocolim}\left(\mathcal{X}(T) \leftarrow \coprod_{1 \leq s \leq n}\left(\mathcal{X}(T) \times U_{s}\right) \rightarrow \coprod_{1 \leq s \leq n}\left(\mathcal{X}(T \cup\{s\}) \times U_{s}\right)\right) .
$$

This can also be described as the union, along $\mathcal{X}(T)$, of the spaces $\mathcal{X}(T) * \mathcal{X}(T \cup\{s\}) U_{s}$.

Clearly $\hat{\mathcal{X}}$ is a functor from $\mathcal{P}(\underline{n}) \times P(\underline{n})^{n}$ to $\mathcal{C}$. We have $\mathcal{X}(T) \cong \hat{\mathcal{X}}(T, \emptyset, \ldots, \emptyset)$, and there is a natural map $\hat{\mathcal{X}}(T, U, \ldots, U) \rightarrow \mathcal{X}(T) *_{Y} U$ that corresponds to the identity map when $U=\emptyset$.

Let $\mathcal{E}$ be the set of all $\left(U_{1}, \ldots, U_{n}\right) \in \mathcal{P}_{0}(\underline{n})^{n}$ such that, for at least one $s \in \underline{n}$, $s \in U_{s}$. Since $\mathcal{E}$ contains the image of the diagonal map $\mathcal{P}_{0}(\underline{n}) \rightarrow \mathcal{P}_{0}(\underline{n})^{n}$, we can factor the map $t_{n-1} F(\mathcal{X}(T))$ as follows:

$$
\begin{aligned}
F(\mathcal{X}(T)) & \rightarrow \operatorname{holim}_{\left(U_{1}, \ldots, U_{n}\right) \in \mathcal{E}} F\left(\hat{\mathcal{X}}\left(T, U_{1}, \ldots, U_{n}\right)\right) \\
& \rightarrow \operatorname{holim}_{U \in \mathcal{P}_{0}(\underline{n})} F(\hat{\mathcal{X}}(T, U, \ldots, U)) \\
& \rightarrow \operatorname{holim}_{U \in \mathcal{P}_{0}(\underline{n})} F\left(\left(\mathcal{X}(T) *_{Y} U\right)\right. \\
& =\left(T_{n-1} F\right)(\mathcal{X}(T)) .
\end{aligned}
$$

We claim that the cube

$$
T \mapsto \underset{\left(U_{1}, \ldots, U_{n}\right) \in \mathcal{E}}{\operatorname{holim}} F\left(\hat{\mathcal{X}}\left(T, U_{1}, \ldots, U_{n}\right)\right)
$$


is cartesian (for any homotopy functor $F$ ).

Let $\mathcal{E}^{*}$ be the set of all $\left(U_{1}, \ldots, U_{n}\right) \in \mathcal{P}_{0}(\underline{n})^{n}$ such that, for some $s, U_{s}=\{s\}$. This poset is left cofinal in $\mathcal{E}$ (exercise; see [10; page 298] for the definition), and it follows that the restriction map from the holim over $\mathcal{E}$ to the holim over $\mathcal{E}^{*}$ is an equivalence. Therefore it is enough if, whenever $\left(U_{1}, \ldots, U_{n}\right) \in \mathcal{E}^{*}$, the cube

$$
T \mapsto F\left(\hat{\mathcal{X}}\left(T, U_{1}, \ldots, U_{n}\right)\right)
$$

is cartesian. In fact, it is cartesian for a very basic reason: if $U_{s_{0}}=\left\{s_{0}\right\}$ then the map

$$
\hat{\mathcal{X}}\left(T, U_{1}, \ldots, U_{n}\right) \rightarrow \hat{\mathcal{X}}\left(T \cup\left\{s_{0}\right\}, U_{1}, \ldots, U_{n}\right)
$$

is an equivalence. To see this, examine the diagram

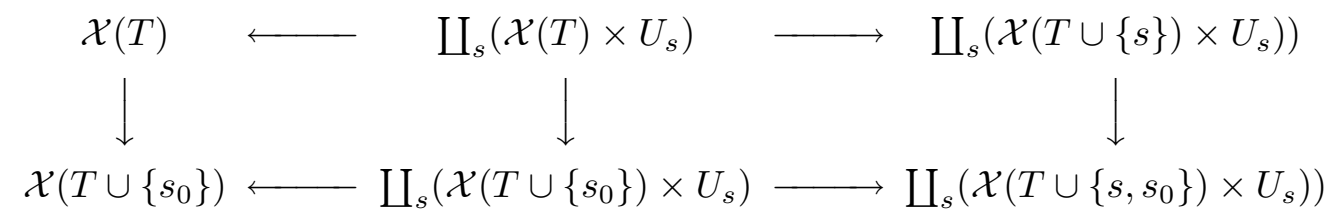

The fact that the induced map from hocolim of top row to hocolim of bottom row is an equivalence follows from the trivial fact that for $s \neq s_{0}$ the square

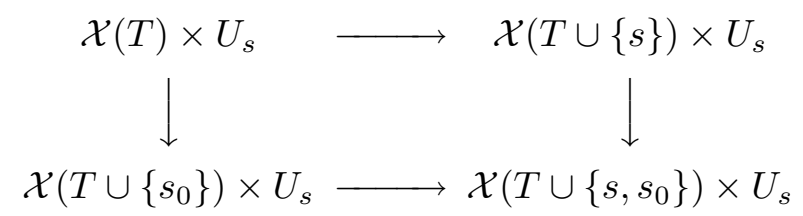

is cocartesian, plus the even more trivial fact that the square

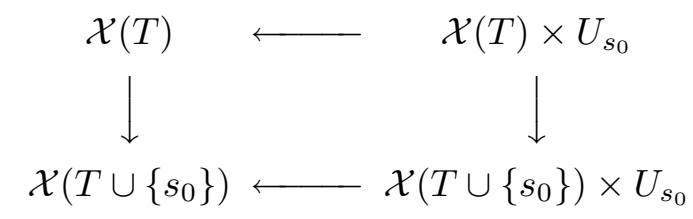

is cocartesian.

1.11 Corollary If $0 \leq m \leq n$ then the map

$$
P_{m} F \stackrel{P_{m}\left(p_{n} F\right)}{\longrightarrow} P_{m} P_{n} F
$$

is an equivalence.

Proof This is formal; using the universal mapping properties of $P_{m}$ and $P_{n}$ and the fact that $m$-excisive functors are $n$-excisive, one sees that $P_{m} P_{n} F$ has the universal mapping property that characterizes $P_{m} F$. 
We now collect the "Taylor polynomials" $P_{n} F$ into a "Taylor series" by showing that there is a natural map $P_{n} F \stackrel{q_{n} F}{\longrightarrow} P_{n-1} F$ satisfying $q_{n} F \circ p_{n} F=p_{n-1} F$. An effortless way to produce $q_{n} F$ as a weak map would be to reason as in the proof of 1.11: $p_{n} F$ is the universal map from $F$ to an $n$-excisive functor and $(n-1)$-excisive functors are $n$-excisive, so there is a unique map $q_{n} F$ in $h \mathcal{F}(\mathcal{C}, \mathcal{D})$ such that $q_{n} F \circ p_{n} F=p_{n-1} F$. On the other hand, it is desirable to define $q_{n} F$ in such a way that $q_{n} F \circ p_{n} F=p_{n-1} F$ on the nose and not just weakly. Moreover, the explicit construction for $q_{n} F$ will be useful in its own right in proving Lemma 2.2.

We will make a (commutative) diagram

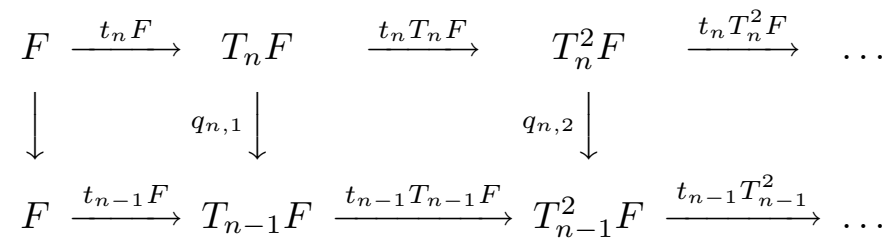

and then define $q_{n} F$ as the induced map of horizontal homotopy colimits. We must define $q_{n, i}$ and then verify that the squares commute.

Notice that $T_{n}^{i} F$ is naturally isomorphic to a homotopy limit indexed by a product of $i$ copies of the partially ordered set $\mathcal{P}_{0}(\underline{n+1})$ :

$$
\left(T_{n}^{i} F\right)(X) \cong \operatorname{holim}_{\left(U_{1}, \ldots, U_{i}\right) \in \mathcal{P}_{0}(\underline{n+1})^{i}} F\left(X *_{Y}\left(U_{1} * \ldots * U_{i}\right)\right)
$$

From this point of view there is an obvious map $T_{n}^{i} F \stackrel{q_{n, i}}{\longrightarrow} T_{n-1}^{i} F$, induced by the inclusion of $\mathcal{P}_{0}(\underline{n})^{i}$ in $\mathcal{P}_{0}(\underline{n+1})^{i}$. The first square in (1.12) now obviously commutes. The $(i+1)^{\mathrm{st}}$ square will commute if both squares commute in

$$
\begin{array}{ccc}
T_{n}^{i} F & \stackrel{t_{n} T_{n}^{i} F}{\longrightarrow} T_{n}^{i+1} F & T_{n}^{i+1} F \\
q_{n, i} F \downarrow & T_{n} q_{n, i} F \downarrow & q_{n, i+1} F \downarrow \\
T_{n-1}^{i} F \underset{t_{n} T_{n-1}^{i} F}{\longrightarrow} & T_{n} T_{n-1}^{i} F \underset{q_{n, 1} T_{n-1}^{i} F}{\longrightarrow} T_{n-1}^{i+1} F
\end{array}
$$

The left square commutes because $t_{n} F$ is natural in $F$. The other commutes because it is induced by a commutative diagram of posets

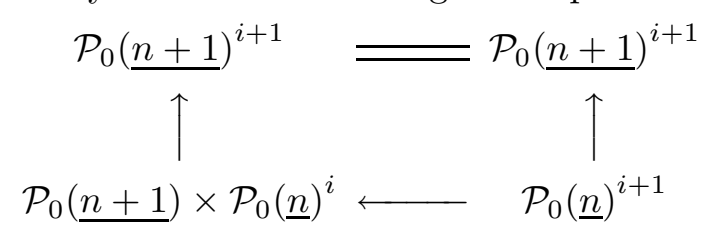

Summing up, we have: 
1.13 Theorem A homotopy functor $F$ from spaces over $Y$ (with or without section) to either spaces, based spaces, or spectra, determines a tower of such functors

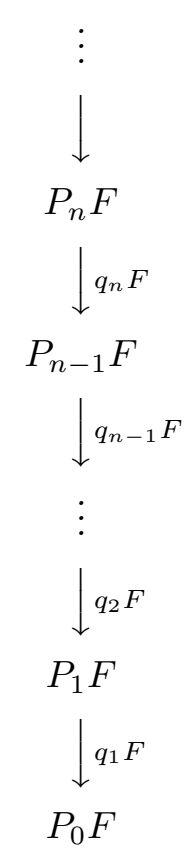

and a map $F \stackrel{\left\{p_{n} F\right\}}{\longrightarrow} \lim _{n} P_{n} F$. Each $P_{n}$ is a homotopy functor (from homotopy functors to homotopy functors), and $p_{n}$ and $q_{n}$ are natural. The functor $P_{n} F$ is always $n$-excisive, and (in the homotopy category of homotopy functors) $p_{n} F$ is universal among maps from $F$ to $n$-excisive functors. If $F$ is $\rho$-analytic and the structural map $X \rightarrow Y$ is $(\rho+1)$-connected, then the connectivity of the map $F(X) \stackrel{p_{n} F}{\longrightarrow}\left(P_{n} F\right)(X)$ tends to $+\infty$ with $n$, so that $F(X)$ is equivalent to the homotopy limit $\left(P_{\infty} F\right)(X \rightarrow Y)$ of the tower.

Proof The last statement is the only new one. Recall from $[10 ; 4.2]$ that $\rho$-analyticity means that there is a number $q$ such that, for all $n \geq 0, F$ satisfies $E_{n}(n \rho-q, \rho+1)$. If $X \in \mathcal{C}$ is such that the map $X \rightarrow Y$ is $k$ connected with $k>\rho$ then by the proof of 1.5 the connectivity of the map $F(X) \stackrel{p_{n} F}{\longrightarrow}\left(P_{n} F\right)(X)$ is at least $(q+k+n(k-\rho))$, which tends to $+\infty$ with $n$.

If $P_{n} F$ is analogous to an $n^{\text {th }}$ Taylor polynomial, then the homotopy fiber

$$
D_{n} F=\operatorname{hofiber}\left(P_{n} F \stackrel{q_{n} F}{\longrightarrow} P_{n-1} F\right)
$$


is analogous to the $n^{\text {th }}$ term in a Taylor series. Notice that the definition of $D_{n} F$ is meaningful if the category $\mathcal{D}$ is either based spaces or spectra, but not if it is unbased spaces. From $P_{n}\left(F \circ \Sigma_{Y}\right) \cong\left(P_{n} F\right) \circ \Sigma_{Y}$ (Remark 1.1) we have

$$
D_{n}\left(F \circ \Sigma_{Y}\right) \cong\left(D_{n} F\right) \circ \Sigma_{Y} \text {. }
$$

1.15 Definition A homotopy functor $F: \mathcal{C} \rightarrow \mathcal{D}$ is called $n$-reduced if $P_{n-1} F \sim *$. It is called $n$-homogeneous, or homogeneous of degree $n$, if it is both $n$-excisive and $n$-reduced.

Thus 1-reduced means reduced, and 1-homogeneous means linear.

1.16 Remark If $n>1$ then it is not easy in general to test whether a functor $F$ is $n$-reduced. Perhaps the main difficulty is that $P_{n-1} F \sim *$ does not imply $T_{n-1} F \sim *$. On the other hand, by 1.6 a sufficient condition for $F$ to be $n$ reduced is that $F$ agrees to order $n-1$ with the constant functor $*$, in other words that the connectivity of $F(X)$ tends to infinity at least $n$ times faster than the connectivity of the map $X \rightarrow Y$. (If $F$ is analytic, or even just stably $n$-excisive, then this condition is also necessary for $F$ to be $n$-reduced.) Thus, for example, for any spectrum $C$ that is bounded below ( $k$-connected for some $k$ ) the $n$-excisive functors $X \mapsto C \wedge X^{\wedge n}$ from based spaces to spectra and $X \mapsto \Omega^{\infty}\left(C \wedge X^{\wedge n}\right)$ from based spaces to based spaces are homogeneous. In fact this holds for all spectra $C$, either by expressing $C$ as a homotopy colimit of bounded-below spectra or by 3.1 below.

1.17 Proposition $D_{n} F$ is always $n$-homogeneous.

Proof The functor $D_{n} F=\operatorname{holim}\left(P_{n} F \rightarrow P_{n-1} F \leftarrow *\right)$ is $n$-excisive because it is a homotopy limit of $n$-excisive functors. To see that it is also $n$-reduced, use 1.7(4) to identify $P_{n-1} D_{n} F$ with the homotopy fiber of the map

$$
P_{n-1}\left(P_{n} F\right) \stackrel{P_{n-1}\left(q_{n} F\right)}{\longrightarrow} P_{n-1}\left(P_{n-1} F\right) ;
$$

by (1.11) this map is an equivalence.

1.18 Proposition Up to equivalence,

(1) $D_{n}$ commutes with finite holim.

(2) $D_{n}$ commutes with hofiber.

(3) $D_{n}$ commutes with filtered hocolim.

(4) for spectrum-valued functors, $D_{n}$ commutes with arbitrary hocolim. 
Proof This follows easily from 1.7.

1.19 Example Let the functors $F_{a}$ and $F_{b}$ be $a$-homogeneous and $b$-homogeneous respectively, with $a<b$, and let $F_{a} \stackrel{f}{\rightarrow} F_{b}$ be any natural map. A simple example is the diagonal inclusion $Q X \rightarrow Q(X \wedge X)$. Then the functor

$$
F(X)=\operatorname{hofiber}\left(F_{a} \stackrel{f}{\rightarrow} F_{b}\right)
$$

is $b$-excisive. Its tower has only two nontrivial layers $D_{a} F \sim F_{a}$ and $D_{b} F \sim$ $\Omega F_{b}$; we have

$$
\begin{aligned}
& P_{n} F \sim * \quad n<a \\
& P_{n} F \sim F_{a} \quad a \leq n<b \\
& P_{n} F \sim F \quad n \leq b
\end{aligned}
$$

All of this follows from $P_{n} F \sim \operatorname{hofiber}\left(P_{n} F_{a} \rightarrow P_{n} F_{b}\right)$. Note that when $a>b$ there can be no interesting natural map $F_{a} \rightarrow F_{b}$, since by (1.8) any such map factors (in $h \mathcal{F}(\mathcal{C}, \mathcal{D}))$ through $P_{a-1} F_{a} \sim *$.

1.20 Example There is a weak equivalence ("Snaith splitting")

$$
\Sigma^{\infty} \Omega \Sigma X \sim \prod_{n \geq 1} \Sigma^{\infty} X^{\wedge n}
$$

for based connected spaces $X$. The functor $\Sigma^{\infty} X^{\wedge n}$ of $X$ is $n$-homogeneous. The $m^{t h}$ Taylor polynomial of the right-hand side of 1.21 is $\prod_{1<n<m} \Sigma^{\infty}\left(X^{\wedge n}\right)$, by 1.6 , since this finite product agrees with the infinite product to order $m$. The same therefore holds for the left-hand side, by 1.1. In particular the $n^{\text {th }}$ homogeneous layer of $\Sigma^{\infty} \Omega \Sigma X$ is $\Sigma^{\infty} X^{\wedge n}$. We will find later that this is enough to determine the $n^{\text {th }}$ homogeneous layer of $F(X)=\Sigma^{\infty} \Omega X$. In fact, using 1.14 we have

$$
\left(D_{n} F\right)(\Sigma X) \sim\left(D_{n}(F \circ \Sigma)\right)(X) \sim \Sigma^{\infty} X^{\wedge n} \sim \Omega^{n} \Sigma^{\infty}(\Sigma X)^{\wedge n},
$$

and by 3.8 this will imply

$$
\left(D_{n} F\right)(X) \sim \Omega^{n} \Sigma^{\infty} X^{\wedge n} .
$$

Incidentally, the naturality of Taylor towers gives a quick way to get from the James model of $\Omega \Sigma X$ to that splitting of $\Sigma^{\infty} \Omega \Sigma X$. The space $J X$, free monoid on the based space $X$, is naturally filtered by word length as an increasing union $*=J_{0} X \subset J_{1} X \subset \ldots$ in such a way that the subquotient $J_{n} / J_{n-1}$ is $X^{\wedge n}$. It follows that for each $n$ there is a natural fibration sequence

$$
\Sigma^{\infty} J_{n-1} X \rightarrow \Sigma^{\infty} J_{n} X \rightarrow \Sigma^{\infty} X^{\wedge n}
$$


One sees by induction that $\Sigma^{\infty} J_{n}$ is $n$-excisive, since it fibers over a (homogeneous) $n$-excisive functor and the fiber is $((n-1)$-excisive, hence) $n$-excisive. The sequence above must split. Indeed, any natural fibration sequence

$$
F(X) \rightarrow G(X) \rightarrow H(X)
$$

of spectra in which $H$ is $n$-homogeneous and $F$ is $(n-1)$-excisive must split: a retraction from $G$ to $F$ (in the homotopy category of homotopy functors) is given by the diagram

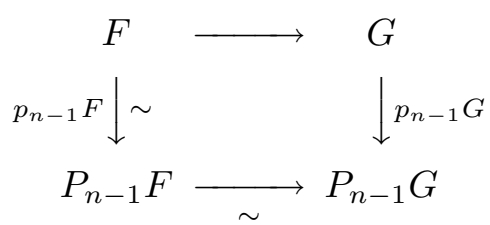

The left arrow is an equivalence because $F$ is $(n-1)$-excisive; the lower arrow because $P_{n-1} H$ is contractible.

1.22 Remark The Taylor tower construction extends easily to functors

$$
\mathcal{C}_{Y_{1}} \times \cdots \times \mathcal{C}_{Y_{n}} \stackrel{F}{\longrightarrow} \mathcal{D}
$$

of several variables. Let us say that $F$ is $\left(d_{1}, \ldots, d_{n}\right)$-excisive [resp. $\left(d_{1}, \ldots, d_{n}\right)$ reduced] if for $1 \leq j \leq n$ it is $d_{j}$-excisive [resp. $d_{j}$-reduced] as a functor of the $j^{t h}$ variable. There is an $n$-variable Taylor polynomial construction which will be denoted $P_{d_{1}, \ldots, d_{n}} F$, and which gives the universal $\left(d_{1}, \ldots, d_{n}\right)$-excisive functor under $F$ (in the homotopy category of homotopy functors). It may be defined either as $P_{d_{1}}^{(1)} \ldots P_{d_{n}}^{(n)} F$ where $P_{d}^{(j)}$ is $P_{d}$ with respect to the $j^{\text {th }}$ variable, or directly as the homotopy colimit of $\left(T_{d_{1}, \ldots, d_{n}}\right)^{k} F$ where

$\left(T_{d_{1}, \ldots, d_{n}} F\right)\left(X_{1}, \ldots, X_{n}\right)=\underset{\left(U_{1}, \ldots, U_{n}\right) \in \prod_{j} \mathcal{P}_{0}\left(\underline{\left.d_{j}+1\right)}\right.}{\operatorname{holim}} F\left(X_{1} *_{Y_{1}} U_{1}, \ldots, X_{n} *_{Y_{n}} U_{n}\right) ;$

these are naturally equivalent. A functor of $n$ variables will be called multilinear if it is linear in each variable, in other words both $(1, \ldots, 1)$-excisive and $(1, \ldots, 1)$-reduced. If $F$ is $(1, \ldots, 1)$-reduced then the functor $P_{1, \ldots, 1} F$ is multilinear and may be called the multilinearization of $F$. Loosely, this is the homotopy colimit of

$$
\Omega^{k_{1}+\ldots k_{n}} F\left(\Sigma_{Y_{1}}^{k_{1}} X_{1}, \ldots, \Sigma_{Y_{n}}^{k_{n}} X_{n}\right)
$$

as $\left(k_{1}, \ldots, k_{n}\right) \rightarrow(\infty, \ldots, \infty)$. 


\section{Delooping homogeneous functors}

We will show that all homogeneous space-valued functors arise from homogeneous spectrum-valued functors. The main step is to show that they have natural deloopings.

Let $\mathcal{H}_{n}(\mathcal{C}, \mathcal{D})$ be the category of homogeneous functors of degree $n$ from $\mathcal{C}$ to $\mathcal{D}$, a full subcategory of $\mathcal{F}(\mathcal{C}, \mathcal{D})$. The homotopy category $\left.h \mathcal{H}_{n}(\mathcal{C}, \mathcal{D})\right)$ is obtained from $\mathcal{H}_{n}(\mathcal{C}, \mathcal{D})$ by formally inverting the (objectwise) equivalences.

The functor $\mathcal{S} p \stackrel{\Omega^{\infty}}{\longrightarrow} \mathcal{T}$ preserves both weak equivalences and cartesian cubes, and therefore composing with it yields a functor

$$
\mathcal{H}_{n}(\mathcal{C}, \mathcal{S} p) \stackrel{\Omega^{\infty}}{\longrightarrow} \mathcal{H}_{n}(\mathcal{C}, \mathcal{T})
$$

which itself takes weak equivalences to weak equivalences.

2.1 Theorem The functor $\mathcal{H}_{n}(\mathcal{C}, \mathcal{S} p) \stackrel{\Omega^{\infty}}{\longrightarrow} \mathcal{H}_{n}(\mathcal{C}, \mathcal{T})$ has an inverse up to weak equivalence.

Proof The key is to get a functor $F \mapsto B F$ from $\mathcal{H}_{n}(\mathcal{C}, \mathcal{T})$ to itself such that $\Omega B F$ is naturally equivalent to $F$. This will be given by the next lemma.

Assume for now that we have this. Then any object $F$ of $\mathcal{H}_{n}(\mathcal{C}, \mathcal{T})$ yields a sequence $\left\{B^{p} F\right\}$ of such objects related by equivalences $B^{p} F \sim \Omega B^{p+1} F$. Call the resulting spectrum-valued functor $B^{\infty} F$. The fact that $B^{\infty} F$ is an object of $\mathcal{H}_{n}(\mathcal{C}, \mathcal{S} p)$ follows easily from the fact that each $B^{p} F$ is an object of $\mathcal{H}_{n}(\mathcal{C}, \mathcal{T})$. The fact that the functor $B^{\infty}$ takes equivalences to equivalences follows from the fact that each functor $B^{p}$ does so. Clearly $\Omega^{\infty} B^{\infty}$ is naturally equivalent to the identity. To check that this is also true for $B^{\infty} \Omega^{\infty}$, let $F$ be an object of $\mathcal{H}_{n}(\mathcal{C}, \mathcal{S} p)$ and write $F(X)=\left\{F_{q}(X)\right\}$. The bispectrum $\left\{B^{p} F_{q}(X)\right\}$ shows that the two spectra

$$
\left\{B^{p} F_{0}(X)\right\}=\left\{B^{p} \Omega^{\infty} F(X)\right\}=B^{\infty} \Omega^{\infty} F(X)
$$

and

$$
\left\{B^{0} F_{q}(X)\right\}=\left\{F_{q}(X)\right\}=F(X)
$$

are naturally equivalent.

The next result provides the desired delooping of $F$, namely $R_{n} F$. As usual $\mathcal{C}_{Y}$ is either $\mathcal{U}_{Y}$ or $\mathcal{T}_{Y}$. 
2.2 Lemma Let $n>0$. If $F: \mathcal{C}_{Y} \rightarrow \mathcal{T}$ is any reduced $(F(Y) \sim *)$ homotopy functor, then up to natural equivalence there is a fibration sequence

$$
P_{n} F \stackrel{q_{n} F}{\longrightarrow} P_{n-1} F \longrightarrow R_{n} F
$$

in which the functor $R_{n} F$ is $n$-homogeneous.

Proof More precisely, we will obtain a natural diagram of homotopy functors

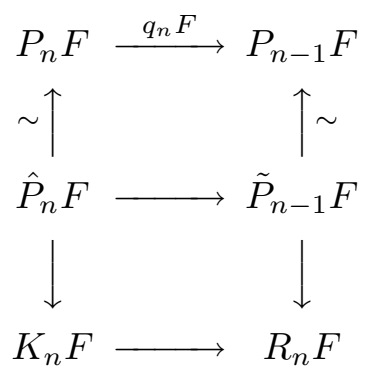

in which the marked arrows are equivalences, the lower square is cartesian, $R_{n} F$ is $n$-homogeneous, and $K_{n} F$ is contractible. (If $F$ were not reduced, then in fact $K_{n} F$ would be equivalent to the constant functor $F(Y)$.)

The proof is based on a close examination of the maps $q_{n, i} F$ which were used in defining $q_{n} F$. The first step is to define, for each $i \geq 0$, a diagram

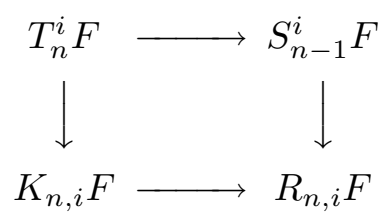

Roughly, this will become the lower square of (2.3) when $i$ goes to infinity.

Define posets

$$
\begin{aligned}
& \mathcal{B}_{n}=\mathcal{P}_{0}(\underline{n+1})-\{\{n+1\}\} \\
& \mathcal{A}_{n, i}=\mathcal{P}_{0}(\underline{n+1})^{i}-\mathcal{P}_{0}(\underline{n})^{i} .
\end{aligned}
$$

Define the functor $S_{n-1} F$ by

$$
\left(S_{n-1} F\right)(X)=\operatorname{holim}_{U \in \mathcal{B}_{n}} F\left(X *_{Y} U\right)
$$

Note that the inclusions $\mathcal{P}_{0}(\underline{n+1}) \supset \mathcal{B}_{n} \supset \mathcal{P}_{0}(\underline{n})$ induce maps

$$
T_{n} F \longrightarrow S_{n-1} F \stackrel{\sim}{\longrightarrow} T_{n-1} F
$$

Geometry $8 \mathcal{G}$ Topology, Volume 7 (2003) 
whose composition is $q_{n, 1} F$. The second map is an equivalence because $\mathcal{P}_{0}(\underline{n})$ is left cofinal in $\mathcal{B}_{n}$. Now let (2.4(i)) be obtained from the posets and inclusions:

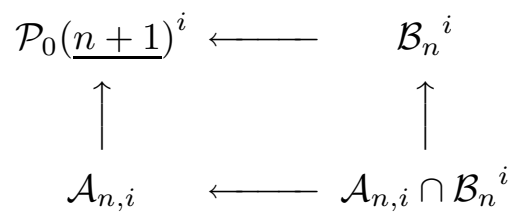

by forming the homotopy limit of $\left(U_{1}, \ldots, U_{i}\right) \mapsto F\left(X *_{Y}\left(U_{1} * \cdots * U_{i}\right)\right)$ over each poset.

Diagram (2.4(i)) is cartesian by [[10]; 1.9], since $\mathcal{A}_{n, i}$ and $B_{n}{ }^{i}$ are concave and their union is $\mathcal{P}_{0}(\underline{n+1})^{i}$.

\subsection{Claim $K_{n, i} F \sim *$.}

Proof Compare $\left(K_{n, i} F\right)(X)$, a holim over $\mathcal{A}_{n, i}$, with the corresponding holim over the smaller poset

$$
\mathcal{A}_{n, i}^{*}=\mathcal{P}_{0}(\underline{n+1})^{i}-\mathcal{B}_{n}{ }^{i}
$$

On the one hand, the comparison map is an equivalence, because $\mathcal{A}_{n, i}^{*}$ is left cofinal in $\mathcal{A}_{n, i}$. On the other hand, the holim of $F\left(X *_{Y}\left(U_{1} * \cdots * U_{i}\right)\right)$ over $\mathcal{A}_{n, i}^{*}$ is contractible: for each $\left(U_{1}, \ldots, U_{i}\right) \in \mathcal{A}_{n, i}^{*}$, we have $\left|U_{j}\right|=1$ for some $j$, so that $U_{1} * \cdots * U_{i}$ is contractible and

$$
F\left(X *_{Y}\left(U_{1} * \cdots * U_{i}\right)\right) \sim F(Y) \sim *
$$

2.8 Claim $R_{n, i} F$ is $n$-reduced.

Proof There is a natural equivalence

$$
P_{n-1} R_{n, i} F \sim R_{n, i} P_{n-1} F
$$

by 1.7 , so it will be enough if $R_{n, i} F$ is contractible whenever $F$ is $(n-1)$ excisive (and reduced).

There is an isomorphism of posets

$$
\mathcal{A}_{n, i} \cap \mathcal{B}_{n}{ }^{i} \cong \mathcal{P}_{0}(\underline{n})^{i} \times \mathcal{P}_{0}(\underline{i})
$$

given by

$$
\begin{aligned}
\left(U_{1}, \ldots, U_{i}\right) & \mapsto\left(V_{1}, \ldots, V_{i}, W\right) \\
V_{i} & =U_{i}-\{n+1\} \\
W & =\left\{i: n+1 \in U_{i}\right\}
\end{aligned}
$$


Therefore $\left(R_{n, i} F\right)(X)$ can be written as

$$
\operatorname{holim}_{\left(V_{1}, \ldots, V_{i}, W\right) \in \mathcal{P}_{0}(\underline{n})^{i} \times \mathcal{P}_{0}(\underline{i})} F\left(X *_{Y}\left(U_{1} * \cdots * U_{i}\right)\right)
$$

Analyze this as an iterated homotopy limit: First fix $\left(V_{2}, \ldots, V_{i}, W\right)$ and take homotopy limit with respect to $V_{1}$. Since $F$ is $(n-1)$-excisive, this yields, up to equivalence, $F\left(X *_{Y}\left(e_{1} * U_{2} * \cdots * U_{i}\right)\right)$ where $e_{1}$ is $\{n+1\}$ or $\emptyset$ according as 1 is or is not in $W$. Next take the homotopy limit with respect to $V_{2}$, then $V_{3}$, and so on through $V_{i}$, obtaining

$$
\left(R_{n, i} F\right)(X) \sim \operatorname{holim}_{W \in \mathcal{P}_{0}(\underline{i})} F\left(X *_{Y}\left(e_{1} * \cdots * e_{i}\right)\right)
$$

This is contractible because for each $W \in \mathcal{P}_{0}(\underline{i})$ the space $e_{1} * \cdots * e_{i}$ is contractible. (It is a simplex of dimension $|W|-1 \geq 0$.)

The next step ought to be to take the homotopy colimit of (2.4(i)) as $i$ tends to $\infty$. This is not possible, since we do not have natural maps $K_{n, i} F \rightarrow K_{n, i+1} F$ or $R_{n, i} F \rightarrow R_{n, i+1} F$. We do, however, have maps defined up to homotopy, and with a little care these will suffice.

Here are two variations on $(2.6(\mathrm{i}+1))$ :

$$
\begin{array}{ccc}
\mathcal{P}_{0}\left(\frac{n+1}{\uparrow}\right)^{i+1} & \longleftarrow & \mathcal{P}_{0}(\underline{n+1}) \times \mathcal{B}_{n}^{i} \\
\uparrow & \mathcal{P}_{0}(\underline{n+1}) \times\left(\mathcal{A}_{n, i} \cap \mathcal{B}_{n}^{i}\right) \\
\mathcal{P}_{0}(\underline{n+1}) \times \mathcal{A}_{n, i} \longleftarrow & \mathcal{P}_{0}(\underline{n+1}) \times \mathcal{B}_{n}^{i} \\
\mathcal{P}_{0}\left(\frac{n+1}{\uparrow}\right)^{i+1} & \longleftarrow \\
\uparrow & \mathcal{A}_{n, i+1} \cap\left(\mathcal{P}_{0}(\underline{n+1}) \times \mathcal{B}_{n}^{i}\right)
\end{array}
$$
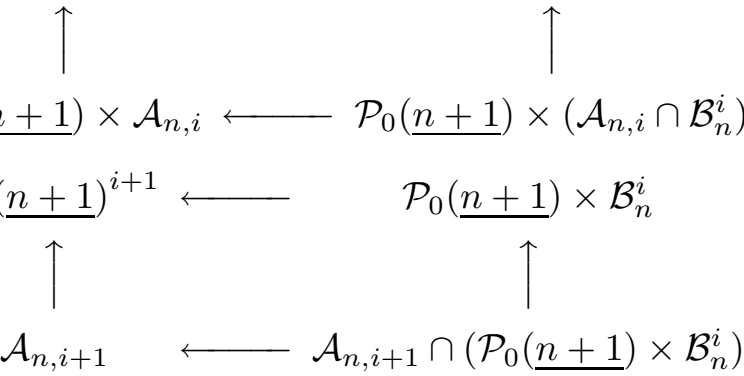$$
(2.10(\mathrm{i}+1))
$$

We have maps of square diagrams of posets

$$
(2.9(i+1)) \rightarrow(2.10(i+1)) \leftarrow(2.6(i+1)) .
$$

From each of the three diagrams we get a square diagram of functors by taking the holim of $F\left(X *_{Y}\left(U_{1} * \cdots * U_{i}\right)\right)$. From $(2.6(\mathrm{i}+1))$ we get $(2.4(\mathrm{i}+1))$. From $(2.9(\mathrm{i}+1))$ we get what might be called $\left.T_{n}(2.4(i))\right)$. From $(2.10(\mathrm{i}+1))$ we get something new; call it $(2.11(\mathrm{i}+1))$. These are related by maps (of square diagrams)

$$
\ldots \rightarrow 2.4(i) \rightarrow T_{n}(2.4(i)) \leftarrow 2.11(i+1) \rightarrow 2.4(i+1) \rightarrow \ldots
$$

Now consider the (pointwise) homotopy colimit of this, another square diagram. This will be the lower half of (2.3). In view of the following, it is essentially a limit of the cartesian squares $2.4(i)$ and therefore it is itself cartesian: 
2.12 Claim The backwards arrow $T_{n}(2.4(i)) \leftarrow 2.11(i+1)$ is an equivalence (in all four corners of the square).

Proof In the upper corners it is an isomorphism. In the lower corners it is induced by the inclusions

$$
\begin{gathered}
\mathcal{P}_{0}(\underline{n+1}) \times \mathcal{A}_{n, i} \longrightarrow \mathcal{A}_{n, i+1} \\
\mathcal{P}_{0}(\underline{n+1}) \times\left(\mathcal{A}_{n, i} \cap \mathcal{B}_{n}^{i}\right) \longrightarrow \mathcal{A}_{n, i+1} \cap\left(\mathcal{P}_{0}(\underline{n+1}) \times \mathcal{B}_{n}^{i}\right)
\end{gathered}
$$

In each of the two cases the larger poset is the union of the smaller one, which is concave (in the sense of [10; page 298]), with the concave set

$$
\mathcal{Q}=\mathcal{A}_{n, 1} \times \mathcal{B}_{n}^{i}
$$

and in each case the intersection is

$$
\mathcal{Q}^{0}=\mathcal{A}_{n, 1} \times\left(\mathcal{A}_{n, i} \cap \mathcal{B}_{n}^{i}\right)
$$

Thus by [10; 0.2] it will be enough if the inclusion $\mathcal{Q}^{0} \rightarrow \mathcal{Q}$ induces an equivalence of holims. But each of these holims is contractible. (The argument is as in the proof of 2.7 ; replace $\mathcal{Q}$ by $\mathcal{A}_{n, 1}^{*} \times \mathcal{B}_{n}^{i}$ and $\mathcal{Q}^{0}$ by $\mathcal{A}_{n, 1}^{*} \times\left(\mathcal{A}_{n, i} \cap \mathcal{B}_{n}^{i}\right)$.)

It follows from 2.7 and 2.12 that $K_{n} F$ is contractible; it is the hocolim of a diagram

$$
\ldots \longrightarrow K_{n, i} F \stackrel{t_{n}}{\longrightarrow} T_{n} K_{n, i} F \stackrel{\sim}{\longleftarrow} ? K_{n, i+1} F \stackrel{t_{n}}{\longrightarrow} \ldots
$$

of contractible objects.

It follows from 2.8 and 2.12 that $R_{n} F$ is $n$-reduced, being the hocolim of the functors

$$
\ldots \longrightarrow R_{n, i} F \stackrel{t_{n}}{\longrightarrow} T_{n} R_{n, i} F \stackrel{\sim}{\longleftarrow} ? R_{n, i+1} F \stackrel{t_{n}}{\longrightarrow} \ldots
$$

Moreover, $R_{n} F$ is $n$-excisive, by Lemma 1.9 ; if $\mathcal{X}$ is any strongly cocartesian $(n+1)$-cube in $\mathcal{C}$ then $\left(R_{n} F\right)(\mathcal{X})$ is cartesian because for each $i$ the map

$$
\left(R_{n, i} F\right)(\mathcal{X}) \stackrel{t_{n}}{\longrightarrow}\left(T_{n} R_{n, i} F\right)(\mathcal{X})
$$

factors through a cartesian cube.

Finally we construct the upper half of (2.3). Note that $\hat{P}_{n} F$ is the hocolim of

$$
\ldots \longrightarrow T_{n}^{i} F \stackrel{t_{n}}{\longrightarrow} T_{n}^{i+1} F \stackrel{=}{\longleftarrow} T_{n}^{i+1} F \stackrel{=}{\longrightarrow} T_{n}^{i+1} F \stackrel{t_{n}}{\longrightarrow} \ldots
$$

Eliminating the identity maps we obtain an equivalence from $\hat{P}_{n} F$ to the hocolim of

$$
\ldots \longrightarrow T_{n}^{i} F \stackrel{t_{n}}{\longrightarrow} T_{n}^{i+1} F \stackrel{t_{n}}{\longrightarrow} \ldots
$$


which is $P_{n} F$. This is the upper left arrow in (2.3). Likewise $\tilde{P}_{n-1} F$ is the hocolim of

$$
\ldots \longrightarrow S_{n-1}^{i} F \stackrel{t_{n}}{\longrightarrow} T_{n} S_{n-1}^{i} F \stackrel{=}{\longleftarrow} T_{n} S_{n-1}^{i} F \longrightarrow S_{n-1}^{i+1} F \stackrel{t_{n}}{\longrightarrow} \ldots
$$

and so has an equivalence to the hocolim of the upper row in

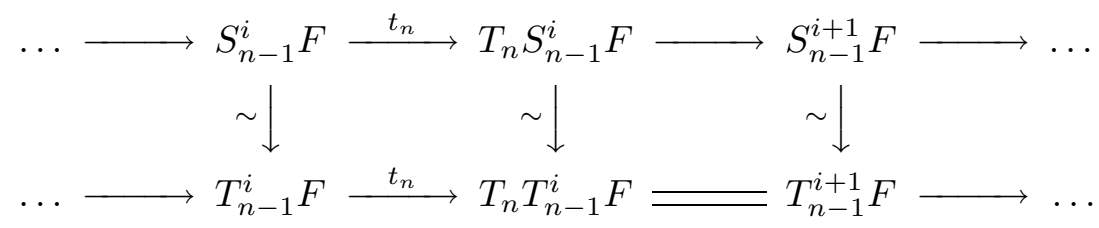

This in turn has an equivalence (2.7) to the hocolim of the lower row, and hence to $P_{n-1} F$. The composed map is the upper right arrow in (2.3). The square commutes. This concludes the proof of (2.2).

\section{Symmetric multilinear functors}

Let $\mathcal{C} \stackrel{\Delta}{\longrightarrow} \mathcal{C}^{n}$ be the diagonal functor. It was shown in $[10 ; 3.4]$ that the composed functor $F \circ \Delta$ is $\left(d_{1}+\cdots+d_{n}\right)$-excisive if the functor $\mathcal{C}^{n} \stackrel{F}{\longrightarrow} \mathcal{D}$ is $d_{j}$-excisive in the $j^{t h}$ variable, or in the language of $1.22\left(d_{1}, \ldots, d_{n}\right)$-excisive. (The latter is easier to write but harder to pronounce.) In particular $F \circ \Delta$ is $n$-excisive if $F$ is $(1, \ldots, 1)$-excisive.

3.1 Lemma If $\mathcal{C}^{n} \stackrel{F}{\longrightarrow} \mathcal{D}$ is a $(1, \ldots, 1)$-reduced homotopy functor, then $F \circ \Delta$ is $n$-reduced. Thus $L \circ \Delta$ is $n$-homogeneous if $\mathcal{C}^{n} \stackrel{L}{\longrightarrow} \mathcal{D}$ is multilinear.

The proof resembles that of 1.8 . The key is:

3.2 Lemma If $\mathcal{C}^{n} \stackrel{F}{\longrightarrow} \mathcal{D}$ is a $(1, \ldots, 1)$-reduced homotopy functor, then for any $X \in \mathcal{C}$ the map

$$
(F \circ \Delta)(X) \stackrel{t_{n-1}(F \circ \Delta)}{\longrightarrow} T_{n-1}(F \circ \Delta)(X)
$$

factors through a (weakly) contractible object.

Proof of 3.1, assuming 3.2 Let $X \in \mathcal{C}$. The object $P_{n-1}(F \circ \Delta)(X)$ is defined as the sequential hocolim of a diagram whose $(i+1)^{\mathrm{st}}$ map is

$$
T_{n-1}^{i}(F \circ \Delta)(X) \stackrel{t_{n-1} T_{n-1}^{i}(F \circ \Delta)}{\longrightarrow} T_{n-1}^{i+1}(F \circ \Delta)(X)
$$

It is enough if each of these maps factors through a weakly contractible object. Lemma 3.2 takes care of this, not only for the first map but also for the others, since the functor $T_{n-1}(F \circ \Delta)$ is equal to $G \circ \Delta$ for a functor $G$ that satisfies the same hypotheses as $F$. 
Proof of 3.2 Let $\mathcal{E}$ and $\mathcal{E}^{*}$ be as in the proof of 1.9. Since $\mathcal{E}$ contains the image of the diagonal map $\mathcal{P}_{0}(\underline{n}) \rightarrow \mathcal{P}_{0}(\underline{n})^{n}$, the map $t_{n-1}(F \circ \Delta)(X)$ can be factored

$$
\begin{aligned}
F(X, \ldots, X) & \cong F\left(X *_{Y} \emptyset, \ldots, X *_{Y} \emptyset\right) \\
& \rightarrow \operatorname{holim}_{\left(U_{1}, \ldots, U_{n}\right) \in \mathcal{E}} F\left(X *_{Y} U_{1}, \ldots, X *_{Y} U_{n}\right) \\
& \rightarrow \operatorname{holim}_{U \in \mathcal{P}_{0}(\underline{n})} F\left(X *_{Y} U, \ldots, X *_{Y} U\right)
\end{aligned}
$$

But

$$
\operatorname{holim}_{\left(U_{1}, \ldots, U_{n}\right) \in \mathcal{E}} F\left(X *_{Y} U_{1}, \ldots, X *_{Y} U_{n}\right) \sim \operatorname{holim}_{\left(U_{1}, \ldots, U_{n}\right) \in \mathcal{E}^{*}} F\left(X *_{Y} U_{1}, \ldots, X *_{Y} U_{n}\right)
$$

and this is a holim of weakly contractible objects: if $\left(U_{1}, \ldots, U_{n}\right) \in \mathcal{E}^{*}$ then some $U_{s}$ is a one-element set, giving $X *_{Y} U_{s} \sim Y$ and

$$
F\left(X *_{Y} U_{1}, \ldots, X *_{Y} U_{n}\right) \sim * .
$$

The functor $L: \mathcal{C}^{n} \rightarrow \mathcal{D}$ is symmetric if it has additional structure consisting of isomorphisms $L(\pi): L\left(X_{1}, \ldots, X_{n}\right) \rightarrow L\left(X_{\pi(1)}, \ldots, X_{\pi(n)}\right)$ for all $\pi \in \Sigma_{n}$, with $L(\sigma \circ \pi)=L(\pi) \circ L(\sigma)$. (In other words $L$ is extended from $\mathcal{C}^{n}$ to a wreath product category.) If $L$ is symmetric and multilinear then the homogeneous functor $L \circ \Delta$ has a $\Sigma_{n}$-action. In the case of spectrum-valued functors (that is, when $\mathcal{D}=\mathcal{S} p$ ), the object

$$
\left(\Delta_{n} L\right)(X)=L(X, \ldots, X)_{h \Sigma_{n}}
$$

is then again an $n$-homogeneous functor of $X$, by 1.7(5), since homotopy orbit space (or spectrum) is a special case of hocolim. We are headed toward proving that all $n$-homogeneous functors arise in just this way.

The inverse of $\Delta_{n}$ will be provided by a construction called the $n^{\text {th }}$ cross-effect, which takes a homotopy functor $F$ and produces a symmetric homotopy functor $c r_{n} F$ of $n$ variables. To see how this inverse construction should go, we recall an algebraic analogue. $\Delta_{n}$ is analogous to the construction in algebra which uses a symmetric multilinear function $l\left(x_{1}, \ldots, x_{n}\right)$ to make a homogeneous function $f(x)=l(x, \ldots, x) / n$ !. For example, if $f$ is a degree two polynomial then from the bilinear form

$$
l\left(x_{1}, x_{2}\right)=f\left(x_{1}+x_{2}\right)-f\left(x_{1}\right)-f\left(x_{2}\right)+f(0)
$$

we recover the purely quadratic part of $f$ as $l(x, x) / 2$. The second cross-effect $\mathrm{cr}_{2} F$ of a functor $F$ of based spaces will take the based spaces $X_{1}$ and $X_{2}$ to the total homotopy fiber of the diagram

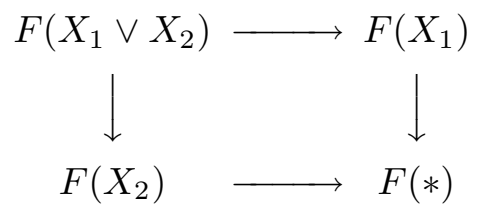

Geometry $8 \mathcal{T}$ Topology, Volume 7 (2003) 
Strictly speaking, in order to make $c r_{2} F$ preserve weak equivalences we must first replace each $X_{i}$ by an equivalent object having nondegenerate basepoint, perhaps by using "whiskers". In the general case (functors of fiberwise based spaces over $Y$ ) the wedge $X_{1} \vee X_{2}$ is replaced by a categorical sum in $\mathcal{T}_{Y}$. Again, before forming the sum we should use fiberwise whiskers, replacing each object $X$ by the mapping cylinder of the structural coretraction $Y \rightarrow X$.

Thus the $n^{\text {th }}$ cross-effect is defined as follows: Let $\mathcal{T}_{Y} \stackrel{F}{\rightarrow} \mathcal{D}$ be a homotopy functor, $\mathcal{D}=\mathcal{T}$ or $\mathcal{S} p$. For objects $\left(X_{1}, \ldots, X_{n}\right)$ of $\mathcal{T}_{Y}$, let $\mathcal{S}\left(X_{1}, \ldots, X_{n}\right)$ be the evident $n$-cube taking $\underline{n}-T$ to the (whiskered) sum, in $\mathcal{T}_{Y}$, of the objects $X_{s}$ for $s \in T$. Define the cross-effect by first applying $F$ to the cube and then taking the total homotopy fiber:

$$
\left(c r_{n} F\right)\left(X_{1}, \ldots, X_{n}\right)=\operatorname{tfiber} F\left(\mathcal{S}\left(X_{1}, \ldots, X_{n}\right)\right) .
$$

It is easy to see that $c r_{n} F$ is a homotopy functor (in each variable), and symmetric and $(1, \ldots, 1)$-reduced.

The first cross-effect is the "reduced functor":

$$
\left(c r_{1} F\right)(X)=\operatorname{fiber}(F(X) \rightarrow F(Y)) .
$$

The $0^{\text {th }}$ cross-effect, should we ever need it, is a functor of no variables: the object $F(Y)$.

3.3 Proposition If $F$ is $n$-excisive then for $0 \leq m \leq n$ the functor $c r_{m+1} F$ is $(n-m)$-excisive in each variable. In particular, the $n^{\text {th }}$ cross-effect of an $n$-excisive functor is symmetric multilinear and the $n^{\text {th }}$ cross-effect of an $(n-1)$ excisive functor is trivial (equivalent to a point).

Proof Induction on $m$. The case $m=0$ is clear. To pass from $m-1$ to $m$, write

$$
\left(c r_{m+1} F\right)\left(X_{1}, \ldots X_{m}, A\right) \cong\left(c r_{m} F_{+A}\right)\left(X_{1}, \ldots, X_{m}\right),
$$

where $F_{+A}(X)=$ hofiber $(F(X+A) \rightarrow F(X))$ and + denotes whiskered sum in $\mathcal{T}_{Y}$. Use the rather obvious fact that $F_{+A}$ is $(n-1)$-excisive if $F$ is $n$-excisive.

In general, then,

$$
\begin{aligned}
c r_{n} D_{n} F & =c r_{n} \text { hofiber }\left(P_{n} F \rightarrow P_{n-1} F\right) \\
& \sim \operatorname{hofiber}\left(c r_{n} P_{n} F \rightarrow c r_{n} P_{n-1} F\right) \\
& \sim c r_{n} P_{n} F .
\end{aligned}
$$

Thus if $F$ is $n$-excisive then $c r_{n} F$ is naturally equivalent to $c r_{n} D_{n} F$; the $n^{\text {th }}$ cross-effect of an $n$-excisive functor "sees" only the $n$-homogeneous part. The following simple result suggests that it sees it quite clearly, at least in the spectrum-valued case. 
3.4 Proposition If $F$ and $G$ are $n$-homogeneous functors $\mathcal{T}_{Y} \rightarrow \mathcal{S} p$, then any (natural) map $F \rightarrow G$ that induces an equivalence $c r_{n} F \rightarrow c r_{n} G$ must be an equivalence itself.

Proof Let $H$ be the homotopy fiber of $F \rightarrow G$. Thus $H$ is $n$-homogeneous and $c r_{n} H$ is the homotopy fiber of $c r_{n} F \rightarrow c r_{n} G$. Since a map of spectra must be an equivalence if its homotopy fiber is contractible, we have only to show that, for an $n$-homogeneous functor $\mathcal{T}_{Y} \stackrel{H}{\longrightarrow} \mathcal{S} p, c r_{n} H \sim *$ implies $H \sim *$. In fact we will do a little better: assuming only that $H$ is $n$-excisive and $c r_{n} H \sim *$, we will show that $H$ is $(n-1)$-excisive.

If $\mathcal{X}$ is any strongly cocartesian $n$-cube in $\mathcal{T}_{Y}$, we must show that $H(\mathcal{X})$ is cartesian. Because we are dealing with spectra, it will be enough if we show that tfiber $H(\mathcal{X}) \sim *$.

By assumption, this holds in the case where $\mathcal{X}$ is the cube $\mathcal{S}\left(X_{1}, \ldots, X_{n}\right)$ for objects $X_{1}, \ldots, X_{n}$ of $\mathcal{T}_{Y}$.

It also holds for the related cube, call it $\mathcal{S}^{*}\left(X_{1}, \ldots, X_{n}\right)$, which has the same objects but with reversed arrows, sending $T$ rather than $\underline{n}-T$ to the sum of the objects $X_{s}$ for $s \in T$. In fact we have

$$
\text { tfiber } \begin{aligned}
H\left(\mathcal{S}^{*}\left(X_{1}, \ldots, X_{n}\right)\right) & \sim \Omega^{n} \text { tfiber } H\left(\mathcal{S}\left(X_{1}, \ldots, X_{n}\right)\right) \\
& =\Omega^{n}\left(c r_{n} H\right)\left(X_{1}, \ldots, X_{n}\right) .
\end{aligned}
$$

This takes care of all cubes $\mathcal{X}$ in which $\mathcal{X}(\emptyset) \sim Y$, because such a cube is naturally equivalent to $\mathcal{S}^{*}(\mathcal{X}(\{1\}), \ldots, \mathcal{X}(\{n\}))$ (see $\left.[10 ; 2.2]\right)$.

Given an arbitrary strongly cocartesian cube $\mathcal{X}$, put

$$
\mathcal{X}^{\prime}(T)=\operatorname{hocolim}(Y \leftarrow \mathcal{X}(\emptyset) \rightarrow \mathcal{X}(T)) .
$$

The obvious map of $n$-cubes $\mathcal{X} \rightarrow \mathcal{X}^{\prime}$ is a strongly cocartesian $(n+1)$-cube, so the resulting cube $H(\mathcal{X}) \rightarrow H\left(\mathcal{X}^{\prime}\right)$ is cartesian. The cube $H\left(\mathcal{X}^{\prime}\right)$ is cartesian by the case already treated. Therefore by $[9 ; 1.6] H(\mathcal{X})$ is cartesian.

Let $\mathcal{L}_{n}(\mathcal{C}, \mathcal{D})$ be the category of symmetric multilinear functors $L: \mathcal{C}^{n} \rightarrow$ $\mathcal{D}$. The maps are the natural maps that respect the symmetry. As usual the homotopy category $h \mathcal{L}_{n}(\mathcal{C}, \mathcal{D})$ means the category obtained by inverting the (objectwise) equivalences.

If $\mathcal{D}$ is either $\mathcal{S} p$ or $\mathcal{T}$ then there is the cross-effect functor

$$
\mathcal{H}_{n}\left(\mathcal{T}_{Y}, \mathcal{D}\right) \stackrel{c r_{n}}{\longrightarrow} \mathcal{L}_{n}\left(\mathcal{T}_{Y}, \mathcal{D}\right)
$$

If $\mathcal{C}$ is either $\mathcal{U}_{Y}$ or $\mathcal{T}_{Y}$ then there is the functor

$$
\mathcal{L}_{n}(\mathcal{C}, \mathcal{S} p) \stackrel{\Delta_{n}}{\longrightarrow} \mathcal{H}_{n}(\mathcal{C}, \mathcal{S} p)
$$

going the other way. Both $c r_{n}$ and $\Delta_{n}$ preserve weak equivalences and so induce functors on homotopy categories. 
3.5 Theorem The functors

$$
\begin{aligned}
& \mathcal{H}_{n}\left(\mathcal{T}_{Y}, \mathcal{S} p\right) \stackrel{c r_{n}}{\longrightarrow} \mathcal{L}_{n}\left(\mathcal{T}_{Y}, \mathcal{S} p\right) \\
& \mathcal{L}_{n}\left(\mathcal{T}_{Y}, \mathcal{S} p\right) \stackrel{\Delta_{n}}{\longrightarrow} \mathcal{H}_{n}\left(\mathcal{T}_{Y}, \mathcal{S} p\right)
\end{aligned}
$$

are mutual inverses up to natural (weak) equivalence.

Proof of 3.5 To prove that the composition

$$
\mathcal{L}_{n}\left(\mathcal{T}_{Y}, \mathcal{S} p\right) \stackrel{\Delta_{n}}{\longrightarrow} \mathcal{H}_{n}\left(\mathcal{T}_{Y}, \mathcal{S} p\right) \stackrel{c r_{n}}{\longrightarrow} \mathcal{L}_{n}\left(\mathcal{T}_{Y}, \mathcal{S} p\right)
$$

is equivalent to the identity, we look to the algebraic analogue. If $l$ is a symmetric multilinear function of $n$ variables and $f$ is the homogeneous polynomial

$$
f(x)=l(x, \ldots, x) / n !,
$$

then $l$ can be recovered from $f$. It is given by

$$
l\left(z_{1}, \ldots, z_{n}\right)=f\left(z_{1}+\cdots+z_{n}\right)-f\left(z_{1} \cdots+z_{n-1}\right)-\cdots+(-1)^{n} f(0)
$$

(an alternating sum of $2^{n}$ terms). One sees this, of course, by expanding the expression

$$
l\left(x_{1}+\cdots+x_{n}, \ldots, x_{1}+\cdots+x_{n}\right)
$$

as a sum of $n^{n}$ terms, cancelling all except the permutations of $l\left(x_{1}, \ldots, x_{n}\right)$ and dividing by $n$ factorial.

Here is a corresponding categorical argument: We have

$$
\begin{aligned}
\left(c r_{n} \Delta_{n} L\right)\left(X_{1}, \ldots, X_{n}\right) & =\operatorname{tfiber}\left(\left(\Delta_{n} L\right) \circ \mathcal{S}\left(X_{1}, \ldots, X_{n}\right)\right) \\
& \cong \operatorname{tfiber}\left(\underline{n}-T \mapsto L\left(\coprod_{s \in T} X_{s}, \ldots, \coprod_{s \in T} X_{s}\right)_{h \Sigma_{n}}\right) \\
& \sim(\operatorname{tfiber}(\mathcal{X}))_{h \Sigma_{n}},
\end{aligned}
$$

where $\mathcal{X}(\underline{n}-T)=L\left(\coprod_{s \in T} X_{s}, \ldots, \coprod_{s \in T} X_{s}\right)$ and $\amalg$ denotes whiskered sum in $\mathcal{T}_{Y}$. The obvious equivalence

$$
\mathcal{X}(\underline{n}-T) \stackrel{\sim}{\longrightarrow} \prod_{\underline{n} \stackrel{\pi}{\longrightarrow} T} L\left(X_{\pi(1)}, \ldots, X_{\pi(n)}\right)
$$

is natural with respect to $T$. It can be interpreted as an equivalence of cubes $\mathcal{X} \rightarrow \prod_{\underline{n} \underset{n}{\rightarrow} \underline{n}} \mathcal{Y}_{\pi}$ where

$$
\begin{aligned}
& \mathcal{Y}_{\pi}(\underline{n}-T)=L\left(X_{\pi(1)}, \ldots, X_{\pi(n)}\right), \quad \text { if } \quad \pi(\underline{n}) \subset T \\
& \mathcal{Y}_{\pi}(\underline{n}-T)=*, \quad \text { otherwise. }
\end{aligned}
$$

For any $\pi$ that is not a permutation and therefore not surjective, the cube $\mathcal{Y}_{\pi}$ is cartesian. (Viewed in one way it is an isomorphism of $(n-1)$-cubes: if 
$s \notin \pi(\underline{n})$ then all maps $\mathcal{Y}_{\pi}(T) \rightarrow \mathcal{Y}_{\pi}(T \cup\{s\})$ are isomorphisms.) For any permutation $\pi$ we have

$$
\operatorname{tfiber}\left(\mathcal{Y}_{\pi}\right) \cong L\left(X_{\pi(1)}, \ldots, X_{\pi(n)}\right)
$$

Therefore

$$
\operatorname{tfiber}(\mathcal{X}) \stackrel{\sim}{\longrightarrow} \prod_{\pi \in \Sigma_{n}} L\left(X_{\pi(1)}, \ldots, X_{\pi(n)}\right)
$$

This map respects the symmetry if the group is made to permute the factors of the right-hand side, and so it leads to an equivalence of homotopy orbit spectra

$$
\left(c r_{n} \Delta_{n} L\right)\left(X_{1}, \ldots, X_{n}\right) \sim\left(\prod_{\pi \in \Sigma_{n}} L\left(X_{\pi(1)}, \ldots, X_{\pi(n)}\right)\right)_{h \Sigma_{n}} \sim L\left(X_{1}, \ldots, X_{n}\right)
$$

Note for future reference that an explicit inverse equivalence

$$
L\left(X_{1}, \ldots, X_{n}\right) \stackrel{\theta}{\rightarrow}\left(c r_{n} \Delta_{n} L\right)\left(X_{1}, \ldots, X_{n}\right)
$$

is the map of total homotopy fibers induced by an obvious map of cubes

$$
\mathcal{Y}_{1} \rightarrow \mathcal{X} \rightarrow \mathcal{X}_{h \Sigma_{n}}
$$

where $\underline{n} \stackrel{1}{\rightarrow} \underline{n}$ is the identity map and the map $\mathcal{Y}_{1}(\emptyset) \rightarrow \mathcal{X}(\emptyset)$ is the map

$$
L\left(X_{1}, \ldots, X_{n}\right) \stackrel{i}{\rightarrow} L(Z, \ldots, Z)
$$

induced by the inclusions $X_{j} \rightarrow Z=\coprod_{1 \leq j \leq n} X_{j}$. The following diagram commutes:

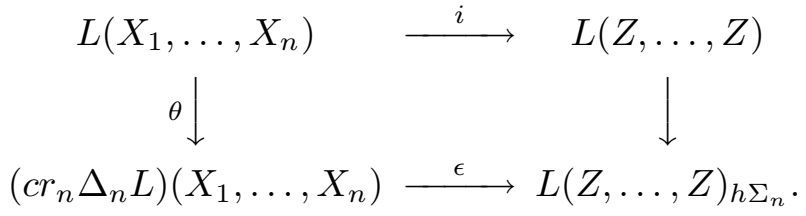

Here, and throughout this proof, $\epsilon$ denotes the projection from the total homotopy fiber of a cubical diagram to the "initial" object in the diagram.

The remaining task is to exhibit an equivalence

$$
\Delta_{n} c r_{n} F \stackrel{\gamma}{\rightarrow} F
$$

for any $n$-homogeneous $\mathcal{T}_{Y} \stackrel{F}{\longrightarrow} \mathcal{S} p$. In fact we will define $\gamma$ for any $n$-excisive $F$ and then show that it induces an equivalence

$$
c r_{n} \Delta_{n} c r_{n} F \stackrel{c r_{n}(\gamma)}{\longrightarrow} c r_{n} F \text {. }
$$

By 3.4 this suffices. 
To define $\gamma$ we use a map $\hat{\gamma}$, defined for $Z \in \mathcal{T}_{Y}$ as the composition

$$
\left(c r_{n} F\right)(Z, \ldots, Z) \stackrel{\epsilon}{\rightarrow} F\left(\coprod_{1 \leq j \leq n} Z\right) \stackrel{F(f)}{\longrightarrow} F(Z)
$$

where $f$ is the "fold" map which takes each copy of $Z$ identically to $Z$. The map $\hat{\gamma}$ is equivariant with respect to the obvious $\Sigma_{n}$-actions. (The action on $F(Z)$ is trivial.) Define $\gamma$ as the composition

$$
\begin{aligned}
\left(\Delta_{n} c r_{n} F\right)(Z) & =\left(\left(c r_{n} F\right)(Z, \ldots, Z)\right)_{h \Sigma_{n}} \\
& \stackrel{\hat{\gamma}_{h \Sigma_{n}}}{\longrightarrow} F(Z)_{h \Sigma_{n}} \cong F(Z) \wedge\left(B \Sigma_{n}\right)_{+} \rightarrow F(Z)
\end{aligned}
$$

where the last arrow is induced by the nontrivial based map $\left(B \Sigma_{n}\right)_{+} \rightarrow S^{0}$.

To see that $\mathrm{cr}_{n}(\gamma)$ is an equivalence we examine the composition

$$
c r_{n} F \stackrel{\theta}{\sim} c r_{n} \Delta_{n} c r_{n} F \stackrel{c r_{n}(\gamma)}{\longrightarrow} c r_{n} F .
$$

It will be enough if it coincides with the identity, at least on the level of homotopy groups. In fact it will be enough if the composition

$$
\left(c r_{n} F\right)\left(X_{1}, \ldots, X_{n}\right) \stackrel{c r_{n}(\gamma) \circ \theta}{\longrightarrow}\left(c r_{n} F\right)\left(X_{1}, \ldots, X_{n}\right) \stackrel{\epsilon}{\longrightarrow} F(Z)
$$

is equal to $\epsilon$, since $\epsilon$ is a split injection. (Here again $Z=\coprod_{1 \leq j \leq n} X_{j}$.)

That it is is a direct consequence of the following facts: The diagram

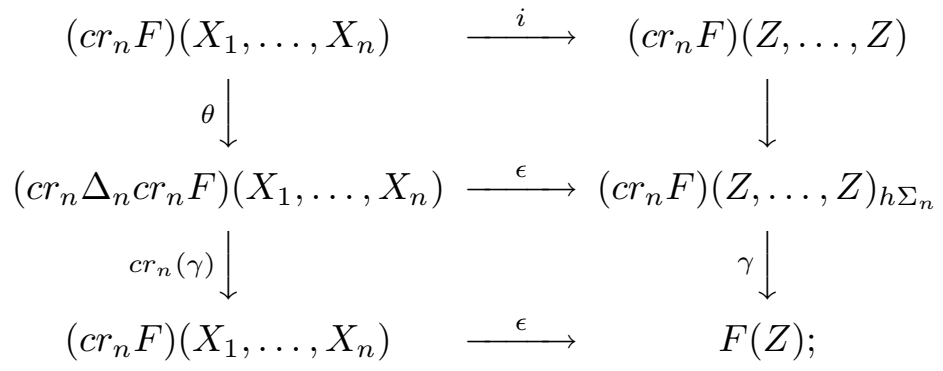

commutes; the composition of the right-hand vertical maps above is $\hat{\gamma}$, which is also the composition of the lower horizontal maps below; the diagram

$$
\begin{array}{ccc}
c r_{n} F\left(X_{1}, \ldots, X_{n}\right) \stackrel{\epsilon}{\longrightarrow} & F(Z) \\
i & F(D) \downarrow \\
c r_{n} F(Z, \ldots, Z) \stackrel{\epsilon}{\longrightarrow} F\left(\coprod_{1 \leq j \leq n} Z\right) \stackrel{F(f)}{\longrightarrow} F(Z),
\end{array}
$$

commutes, where $Z \stackrel{D}{\rightarrow} \coprod_{1 \leq j \leq n} Z$ sends the copy of $X_{i}$ in $Z$ to the copy of $X_{i}$ in the $i^{t h}$ copy of $Z$; and the composition $f \circ D$ is the identity map. 
3.6 Corollary The functor $\mathcal{H}_{n}\left(\mathcal{T}_{Y}, \mathcal{T}\right) \stackrel{c r_{n}}{\longrightarrow} \mathcal{L}_{n}\left(\mathcal{T}_{Y}, \mathcal{T}\right)$ has an inverse up to weak equivalence.

Proof of 3.6 Using the commutative diagram

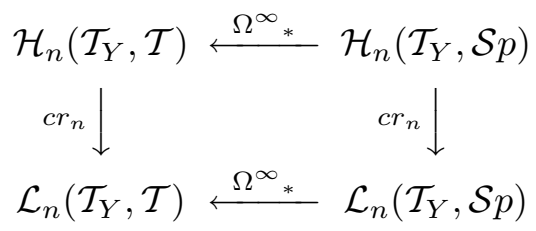

this will follow from 3.5, 2.1, and the next result.

3.7 Proposition The functor $\mathcal{L}_{n}(\mathcal{C}, \mathcal{S} p) \stackrel{\Omega^{\infty}}{\longrightarrow} \mathcal{L}_{n}(\mathcal{C}, \mathcal{T})$ has an inverse up to weak equivalence.

Proof As in proving 2.1, we need a delooping functor from $\mathcal{L}_{n}(\mathcal{C}, \mathcal{T})$ to itself. This is much easier than 2.2 .

We refer to Remark 1.22 for notation. If $L$ is symmetric multilinear then there are natural equivalences

$$
L\left(X_{1}, \ldots, X_{n}\right) \stackrel{\sim}{\longrightarrow}\left(T_{1, \ldots, 1} L\right)\left(X_{1}, \ldots, X_{n}\right) \stackrel{\sim}{\longleftarrow} \Omega^{n} L\left(\Sigma_{Y} X_{1}, \ldots, \Sigma_{Y} X_{n}\right) .
$$

We will need these equivalences to respect the $\Sigma_{n}$-symmetry. In order for this to be true, permutations of the loop coordinates must be built into the symmetry of the last expression; a better expression is $\Omega^{V_{n}} L\left(\Sigma_{Y} X_{1}, \ldots, \Sigma_{Y} X_{n}\right)$, where $V_{n}$ is the standard $n$-dimensional representation of $\Sigma_{n}$. Since this contains a trivial one-dimensional representation, we have what we need: if $V_{n}=\mathbb{R} \oplus \bar{V}_{n}$ then $L \sim \Omega B L$ where $B L$ is defined by

$$
(B L)\left(X_{1}, \ldots, X_{n}\right)=\Omega^{\bar{V}_{n}} L\left(\Sigma_{Y} X_{1}, \ldots, \Sigma_{Y} X_{n}\right) .
$$

We can now justify an assertion made in 1.20 . Let $\mathcal{D}$ be either $\mathcal{T}$ or $\mathcal{S} p$.

3.8 Corollary If $\mathcal{T}_{Y} \stackrel{F}{\longrightarrow} \mathcal{D}$ is $n$-homogeneous then $F$ is determined by $F \circ \Sigma_{Y}$.

Proof According to 3.5 and $3.6 F$ is determined by the cross-effect $c r_{n} F$, and this satisfies

$$
\left(c r_{n} F\right)\left(\Sigma_{Y} X_{1}, \ldots, \Sigma_{Y} X_{n}\right) \cong\left(c r_{n}\left(F \circ \Sigma_{Y}\right)\right)\left(X_{1}, \ldots, X_{n}\right) .
$$

On the other hand, for any symmetric multilinear functor $L$ we have

$$
L\left(X_{1}, \ldots, X_{n}\right) \sim \Omega^{V_{n}} L\left(\Sigma_{Y} X_{1}, \ldots, \Sigma_{Y} X_{n}\right),
$$

so that

$$
\begin{aligned}
\left(c r_{n} F\right)\left(X_{1}, \ldots, X_{n}\right) \sim \Omega^{V_{n}}\left(c r_{n} F\right)\left(\Sigma_{Y} X_{1}, \ldots, \Sigma_{Y} X_{n}\right) \\
\\
\cong \Omega^{V_{n}} c r_{n}\left(F \circ \Sigma_{Y}\right)\left(X_{1}, \ldots, X_{n}\right)
\end{aligned}
$$




\section{The role of the base point}

The cross-effect construction applies to functors $\mathcal{T}_{Y} \rightarrow \mathcal{D}$ but not to functors $\mathcal{U}_{Y} \rightarrow \mathcal{D}$. In spite of this we now show that the classification of homogeneous functors extends without change to the $\mathcal{U}_{Y}$ case.

Let $\mathcal{T}_{Y} \stackrel{\phi}{\longrightarrow} \mathcal{U}_{Y}$ be the forgetful functor. Because $\phi$ preserves equivalences and cocartesian square diagrams, composition with $\phi$ yields functors

$$
\begin{gathered}
\mathcal{H}_{n}\left(\mathcal{U}_{Y}, \mathcal{D}\right) \stackrel{\phi^{*}}{\longrightarrow} \mathcal{H}_{n}\left(\mathcal{T}_{Y}, \mathcal{D}\right) \\
\mathcal{L}_{n}\left(\mathcal{U}_{Y}, \mathcal{D}\right) \stackrel{\phi^{*}}{\longrightarrow} \mathcal{L}_{n}\left(\mathcal{T}_{Y}, \mathcal{D}\right) .
\end{gathered}
$$

4.1 Theorem If $\mathcal{D}$ is $\mathcal{T}$ or $\mathcal{S} p$ then both of the two functors $\phi^{*}$ above have inverses up to weak equivalence.

4.2 Corollary The functor $\mathcal{L}_{n}\left(\mathcal{U}_{Y}, \mathcal{S} p\right) \stackrel{\Delta_{n}}{\longrightarrow} \mathcal{H}_{n}\left(\mathcal{U}_{Y}, \mathcal{S} p\right)$ has an inverse up to weak equivalence.

Proof of 4.2 Use 3.5, 4.1, and the diagram

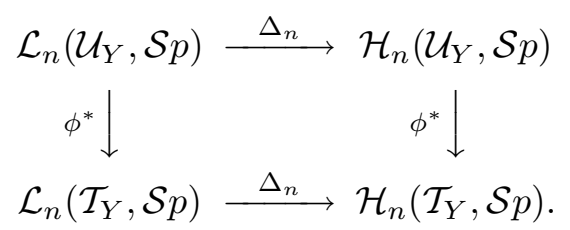

Proof of 4.1 We can assume $\mathcal{D}=\mathcal{S} p$, since by 2.1 and 3.7 the spectrumvalued case of 4.1 implies the space-valued case.

Let $\mathcal{U}_{Y} \stackrel{\psi}{\rightarrow} \mathcal{T}_{Y}$ be the left adjoint of $\phi$, so that if $X$ is a space over $Y$ then $\psi(X)$ is the disjoint union of $X$ and $Y$ viewed as a fiberwise based space over $Y$. Like $\phi$, this functor preserves equivalences and cocartesian square diagrams. Therefore if $\mathcal{T}_{Y} \stackrel{F}{\longrightarrow} \mathcal{S} p$ is $n$-excisive then $\mathcal{U}_{Y} \stackrel{\psi^{*} F}{\longrightarrow} \mathcal{S} p$ will be $n$-excisive. Unlike $\phi, \psi$ does not preserve the final object $Y$, so $\psi^{*} F$ need not be homogeneous if $F$ is. We will show that

$$
\mathcal{H}_{n}\left(\mathcal{T}_{Y}, \mathcal{S} p\right) \stackrel{D_{n} \circ \psi^{*}}{\longrightarrow} \mathcal{H}_{n}\left(\mathcal{U}_{Y}, \mathcal{S} p\right)
$$

is an inverse, up to weak equivalence, for

$$
\mathcal{H}_{n}\left(\mathcal{U}_{Y}, \mathcal{S} p\right) \stackrel{\phi^{*}}{\longrightarrow} \mathcal{H}_{n}\left(\mathcal{T}_{Y}, \mathcal{S} p\right)
$$


The unit map $X \rightarrow \phi \psi X$ of the adjoint pair induces a map $F \rightarrow \psi^{*} \phi^{*} F$. For any morphism $X \rightarrow X^{\prime}$ in $\mathcal{U}_{Y}$ the square diagram

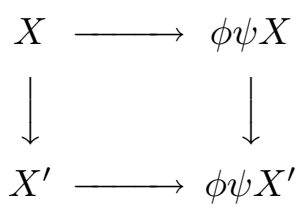

is cocartesian. Therefore if $\mathcal{X}$ is any strongly cocartesian $n$-cube in $\mathcal{U}_{Y}$ the unit yields a strongly cocartesian $(n+1)$-cube

$$
\mathcal{X} \rightarrow \phi \psi \mathcal{X} \text {. }
$$

It follows that if $\mathcal{U}_{Y} \stackrel{F}{\longrightarrow} \mathcal{S} p$ is $n$-excisive then the fiber of $F \rightarrow \psi^{*} \phi^{*} F$ is $(n-1)$-excisive. Thus if $F$ is $n$-homogeneous there are natural equivalences

$$
F \stackrel{\sim}{\longrightarrow} P_{n} F \stackrel{\sim}{\longleftarrow} D_{n} F \stackrel{\sim}{\longrightarrow} D_{n} \psi^{*} \phi^{*} F
$$

(The last map is an equivalence because its fiber is contractible. This implication relies on the fact that these are spectrum-valued functors.)

The counit $\psi \phi X \rightarrow X$, like the unit, yields a cocartesian square for every map and a strongly cocartesian $(n+1)$-cube for every strongly cocartesian $n$-cube. Therefore for any $n$-homogeneous $\mathcal{U}_{Y} \stackrel{F}{\longrightarrow} \mathcal{S} p$ it yields equivalences

$$
F \stackrel{\sim}{\longrightarrow} P_{n} F \stackrel{\sim}{\longleftarrow} D_{n} F \stackrel{\sim}{\longleftarrow} D_{n} \phi^{*} \psi^{*} F \cong \phi^{*} D_{n} \psi^{*} F .
$$

This completes the proof in the homogeneous case.

We sketch the proof in the multilinear case, which is much the same. The inverse of

$$
\mathcal{L}_{n}\left(\mathcal{U}_{Y}, \mathcal{S} p\right) \stackrel{\phi^{*}}{\longrightarrow} \mathcal{L}_{n}\left(\mathcal{T}_{Y}, \mathcal{S} p\right)
$$

is

$$
\mathcal{L}_{n}\left(\mathcal{T}_{Y}, \mathcal{S} p\right) \stackrel{r \circ \psi^{*}}{\longrightarrow} \mathcal{L}_{n}\left(\mathcal{U}_{Y}, \mathcal{S} p\right)
$$

where $r$ is the operation of "reducing" a symmetric homotopy functor of $n$ variables in all variables simultaneously. For example, when $n=2$ then $(r L)\left(X_{1}, X_{2}\right)$ is the total homotopy fiber of

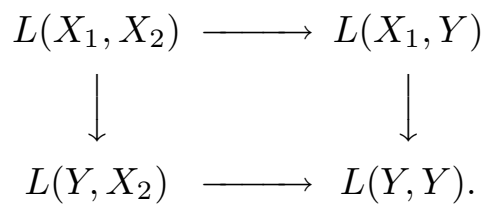

The key point is that the maps $L \rightarrow \phi^{*} \psi^{*} L$ and $\psi^{*} \phi^{*} L \rightarrow L$ induce equivalences $r L \rightarrow r \phi^{*} \psi^{*} L \cong \phi^{*} r \psi^{*} L$ and $r \psi^{*} \phi^{*} L \rightarrow r L$. For example, in the composition

$$
L\left(X_{1}, X_{2}\right) \rightarrow L\left(X_{1}, \psi \phi X_{2}\right) \rightarrow L\left(\psi \phi X_{1}, \psi \phi X_{2}\right)
$$


the fiber of the first map becomes contractible upon reducing with respect to $X_{2}$ while the fiber of the second map becomes contractible upon reducing with respect to $X_{1}$, and so they both become contractible upon applying $r$.

The proof of 4.1 suggests a variant of the notion of multilinear functor, related to it as unreduced homology is related to reduced homology.

4.3 Definition A functor $\mathcal{U}_{Y}^{n} \stackrel{L}{\rightarrow} \mathcal{S} p$ is unreduced-multilinear if it is 1-excisive in each variable and if it satisfies $L\left(X_{1}, \ldots, X_{n}\right) \sim *$ whenever $X_{s}$ is the initial object $\emptyset$ for some $s$. The category of symmetric unreduced-multinear functors is $\hat{\mathcal{L}}_{n}\left(\mathcal{U}_{Y}, \mathcal{S} p\right)$.

Since $\psi(\emptyset) \cong Y$, the functor $\psi^{*}$ maps $\mathcal{L}_{n}\left(\mathcal{T}_{Y}, \mathcal{S} p\right)$ into $\hat{\mathcal{L}}_{n}\left(\mathcal{U}_{Y}, \mathcal{S} p\right)$.

4.4 Proposition The functor $\mathcal{L}_{n}\left(\mathcal{T}_{Y}, \mathcal{S} p\right) \stackrel{\psi^{*}}{\longrightarrow} \hat{\mathcal{L}}_{n}\left(\mathcal{U}_{Y}, \mathcal{S} p\right)$ has an inverse up to weak equivalence.

Proof The proof of 4.1 shows that $\phi^{*} \circ r$ is an inverse.

4.5 Warning Although Definition 4.3 could be extended verbatim to functors into based spaces, the resulting category $\hat{\mathcal{L}}_{n}\left(\mathcal{U}_{Y}, \mathcal{T}\right)$ would not have the expected property: the corresponding variant of Proposition 4.4 would be false. An instructive example is obtained by adding a disjoint basepoint to the excisive functor $\mathcal{U} \stackrel{P_{1} J}{\longrightarrow} \mathcal{U}$ mentioned near the end of the introduction.

4.6 Remark 3.8 is valid for functors of unbased spaces, in view of 4.1 and the fact that $\phi$ commutes with $\Sigma_{Y}$ up to isomorphism.

\section{The $n^{t h}$ differential and the $n^{t h}$ derivative}

We can summarize the main results of Sections 2 through 4 by saying that the following eight categories of functors are equivalent at the homotopy category level:

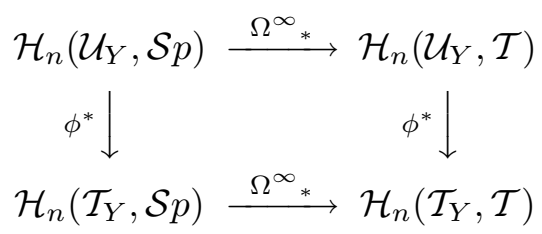

Geometry 85 Topology, Volume 7 (2003) 


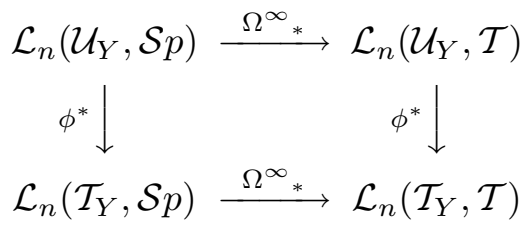

In addition to the arrows displayed, there is also $c r_{n}$ from the upper square to the lower in each of the two $\left(\mathcal{T}_{Y},-\right)$ cases and $\Delta_{n}$ from the lower square to the upper in each of the $(-, \mathcal{S} p)$ cases. We have explicitly inverted enough of these arrows to show that all of them are invertible: We inverted the four called $\Omega_{*}^{\infty}$ in 2.1 and 3.7 ; we inverted the left hand $\phi^{*}$ of each square in 4.1 ; and in 3.1 we connected the two squares by showing that $c r_{n}$ and $\Delta_{n}$ are inverses in the $\left(\mathcal{T}_{Y}, \mathcal{S} p\right)$ case.

Let $\mathcal{C}_{Y} \stackrel{F}{\longrightarrow} \mathcal{D}$ be a homotopy functor from spaces over $Y$ to either based spaces or spectra, and suppose that we wish to describe the homogeneous layer $D_{n} F$ in its Taylor tower for some $n \geq 1$. By the results above, knowing $D_{n} F$ is the same as knowing a certain symmetric multilinear functor. This will be called the $n$-fold differential of $F$.

5.1 Definition The object in $\mathcal{L}_{n}\left(\mathcal{C}_{Y}, \mathcal{D}\right)$ corresponding to the homogeneous functor $D_{n} F \in \mathcal{H}_{n}\left(\mathcal{C}_{Y}, \mathcal{D}\right)$ is called the $n$-fold differential of $F$ and is denoted by $D^{(n)} F$.

Specifically, $D^{(n)} F$ determines $D_{n} F$ by the rule:

$$
\left(D_{n} F\right)(X) \sim\left(D^{(n)} F\right)(X, \ldots, X)_{h \Sigma_{n}}
$$

in the case when $\mathcal{D}=\mathcal{S} p$, or

$$
\left(D_{n} F\right)(X) \sim \Omega^{\infty}\left(\left(B^{\infty} D^{(n)} F\right)(X, \ldots, X)_{h \Sigma_{n}}\right)
$$

in the case when $\mathcal{D}=\mathcal{T}$. Here $B^{\infty}$ is the inverse (up to natural weak equivalence) to

$$
L_{n}\left(\mathcal{C}_{Y}, \mathcal{S} p\right) \stackrel{\Omega_{*}^{\infty}}{\longrightarrow} L_{n}\left(\mathcal{C}_{Y}, \mathcal{T}\right)
$$

provided by 3.6.

Conversely, to obtain $D^{(n)} F$ from $D_{n} F$ one simply takes the $n^{\text {th }}$ cross-effect in the case when $\mathcal{C}_{Y}=\mathcal{T}_{Y}$, while in the case when $\mathcal{C}_{Y}=\mathcal{U}_{Y}$ one knows that $\phi^{*} D^{(n)} F$ determines $D^{(n)} F$ by 4.2 and is given by

$$
\phi^{*} D^{(n)} F \cong D^{(n)} \phi^{*} F=c r_{n} D_{n} \phi^{*} F .
$$

In practice we often work with a functor $\mathcal{U}_{Y} \stackrel{F_{Y}}{\longrightarrow} \mathcal{D}$ that is the restriction of a functor $\mathcal{U} \stackrel{F}{\longrightarrow} \mathcal{D}$, meaning the composition of $F$ with the forgetful functor $\mathcal{U}_{Y} \rightarrow \mathcal{U}$. 
5.2 Definition In this case $D^{(n)} F_{Y}$ is called the $n$-fold differential of $F$ at $Y$ and denoted by $D_{Y}^{(n)} F$.

The next theme to be developed is the description of multilinear functors by their "coefficients". We discuss this briefly in the important special case when $Y$ is the one-point space, and then a little more elaborately in the general case.

If $C$ is a spectrum then the functor

$$
L\left(X_{1}, \ldots, X_{n}\right)=C \wedge\left(X_{1} \wedge \cdots \wedge X_{n}\right)
$$

from ( $n$-tuples of) based spaces to spectra is multilinear, and if $C$ has an action of the symmetric group $\Sigma_{n}$ then $L$ is symmetric.

Conversely, if $L$ is a multilinear functor from based spaces to spectra then, taking $C$ to be $L\left(S^{0}, \ldots, S^{0}\right)$, we have (essentially, see [9; page 5]) a natural assembly map

$$
C \wedge\left(X_{1} \wedge \cdots \wedge X_{n}\right) \rightarrow L\left(X_{1}, \ldots, X_{n}\right) .
$$

This is an equivalence when $X_{j}=S^{0}$ for all $j$, and it follows (see 5.8 below), using the multilinearity of both functors, that it is an equivalence when the $X_{j}$ are finite complexes. If $L$ satisfies a suitable limit axiom ( 5.10 below), then this even holds for all $X_{j}$. If $L$ is symmetric then $C$ gets a $\Sigma_{n}$-action and the assembly map respects the symmetry.

In short, symmetric multilinear functors from finite based spaces to spectra correspond precisely, in the sense of an equivalence of homotopy categories, to spectra with $\Sigma_{n}$-action. The spectrum (together with its $\Sigma_{n}$-action) is called the coefficient of the symmetric multilinear functor, or of the corresponding homogeneous functor.

Thus if $F$ is a homotopy functor from based spaces to based spaces or spectra then for every $n>0$ the $n$-homogeneous layer $D_{n} F$ of its Taylor tower is governed by a certain spectrum with $\Sigma_{n}$-action. This will be called the $n^{t h}$ derivative of $F$ at the one-point space and denoted by $\partial^{(n)} F(*)$. In the spectrum-valued case we have

$$
\partial^{(n)} F(*) \simeq\left(D^{(n)} F\right)\left(S^{0}, \ldots, S^{0}\right)
$$

and

$$
\left(D_{n} F\right)(X) \simeq\left(\partial^{(n)} F(*) \wedge X^{\wedge n}\right)_{h \Sigma_{n}} .
$$

In the space-valued case we have

$$
\Omega^{\infty} \partial^{(n)} F(*) \simeq\left(D^{(n)} F\right)\left(S^{0}, \ldots, S^{0}\right)
$$

and

$$
\left(D_{n} F\right)(X) \simeq \Omega^{\infty}\left(\left(\partial^{(n)} F(*) \wedge X^{\wedge n}\right)_{h \Sigma_{n}}\right) .
$$


To fully describe $\partial^{(n)} F(*)$ in the space-valued case, we examine the proof of 3.7 and see that the $i^{\text {th }}$ space in the spectrum is

$$
\Omega^{i \bar{V}_{n}}\left(D^{(n)} F\right)\left(S^{i}, \ldots, S^{i}\right)
$$

5.3 Remark By the proof of 3.8 we have

$$
\partial^{(n)} F(*) \sim \Omega^{V_{n}} \partial^{(n)}(F \circ \Sigma)(*)
$$

5.4 Remark If a subgroup $G \subset \Sigma_{n}$ acts on a spectrum $C$ then the functor $\left(C \wedge X^{\wedge n}\right)_{h G}$ is homogeneous. When written in standard form it is

$$
\left(\left(\left(\Sigma_{n}\right)_{+} \wedge_{G} C\right) \wedge X^{\wedge n}\right)_{h \Sigma_{n}}
$$

the coefficient is the "induced spectrum" $\left(\Sigma_{n}\right)_{+} \wedge_{G} C$.

We now pass from functors of spaces to functors of spaces over $Y$ while doing our best to retain the principle that a multilinear functor is determined by its behavior on a small class of objects.

Here is some notation for naming special objects of $\mathcal{T}_{Y}$ : If $Z$ is a based space and $y$ is a point in $Y$ then let $Y \vee_{y} Z$ be the union of $Y$ and $Z$ with $y$ identified to the basepoint of $Z$. This is to be viewed as a space over $Y$ with all of $Z$ being mapped to $y$. More generally if several points $y_{1}, \ldots, y_{n}$ and several based spaces $Z_{1}, \ldots, Z_{n}$ are given then $Y \vee_{y_{1}} Z_{1} \vee \cdots \vee_{y_{n}} Z_{n}$ is the space obtained from $Y$ by attaching $Z_{j}$ at $y_{j}$ for all $j$. Again this is to be viewed as an object of $\mathcal{T}_{Y}$.

Let $F$ be a homotopy functor from unbased spaces to either based spaces or spectra.

5.6 Definition Let $Y$ be a space and let $y_{1}, \ldots, y_{n}$ be points in $Y$. The $n^{\text {th }}$ derivative of $F$ at $\left(Y, y_{1}, \ldots, y_{n}\right)$, denoted

$$
\partial_{y_{1}, \ldots, y_{n}}^{(n)} F(Y)
$$

is the coefficient spectrum of the multilinear functor

$$
\left(Z_{1}, \ldots, Z_{n}\right) \mapsto\left(D_{Y}^{(n)} F\right)\left(Y \vee_{y_{1}} Z_{1}, \ldots, Y \vee_{y_{n}} Z_{n}\right)
$$


Thus in the spectrum-valued case we have

$$
\partial_{y_{1}, \ldots, y_{n}}^{(n)} F(Y) \simeq\left(D_{Y}^{(n)} F\right)\left(Y \vee_{y_{1}} S^{0}, \ldots, Y \vee_{y_{n}} S^{0}\right),
$$

$\partial^{(n)} F$ has a symmetry with respect to permutations of $\left(y_{1}, \ldots, y_{n}\right)$ and is also functorial in $Y$. To be more precise, we have here a functor whose domain is the category in which an object is a "space with $n$ basepoints" $\left(Y, y_{1}, \ldots, y_{n}\right)$ and a morphism $\left(Y, y_{1}, \ldots, y_{n}\right) \rightarrow\left(W, w_{1}, \ldots, w_{n}\right)$ is a pair $(f, \pi)$ with $Y \stackrel{f}{\rightarrow} W$ being a continuous map and $\pi$ a permutation such that $f\left(y_{\pi(j)}\right)=w_{j}$ for all $j$. The points $y_{j}$ are not assumed distinct.

It is true in various senses, beginning with 5.9 below, that the $n^{\text {th }}$ derivative of a functor determines the behavior of the $n^{\text {th }}$ differential, at least on finite objects.

5.7 Definition An object $Y \stackrel{i}{\rightarrow} X \stackrel{r}{\rightarrow} Y$ of $\mathcal{T}_{Y}$ is finite if $(X, i(Y))$ is a finite CW pair. An object $X \stackrel{r}{\rightarrow} Y$ of $\mathcal{U}_{Y}$ is finite if the space $X$ is finite CW.

5.8 Proposition Let $L_{1} \stackrel{g}{\rightarrow} L_{2}$ be a map between symmetric multilinear functors from $\mathcal{T}_{Y}$ to $\mathcal{S} p$. In order that

$$
L_{1}\left(X_{1}, \ldots, X_{n}\right) \stackrel{g}{\rightarrow} L_{2}\left(X_{1}, \ldots, X_{n}\right)
$$

should be an equivalence whenever the objects $X_{j}$ are finite, it is enough if $g$ is an equivalence in the special case when each $X_{j}$ is $Y \vee y_{j} S^{0}$ for an arbitrary point $y_{j} \in Y$.

Proof The symmetry is irrelevant here, and it is clear that the case $n=1$ implies the general case. We therefore give a proof for the case of a map $L_{1} \stackrel{g}{\rightarrow} L_{2}$ between linear functors from $\mathcal{T}_{Y}$ to spectra.

Define $\mathcal{T}_{Y} \stackrel{L}{\longrightarrow} \mathcal{S} p$ by letting $L(X)$ be the homotopy fiber of $L_{1}(X) \stackrel{g}{\rightarrow} L_{2}(X)$. The functor $L$ "vanishes" at $Y \vee_{y} S^{0}$, for every point $y$ in $Y$, in the sense that $L\left(Y \vee_{y} S^{0}\right) \simeq *$. We also know that $L$ is linear; it preserves weak equivalences, it takes cocartesian squares to cartesian squares, and it vanishes at $Y$. We must show that $L$ vanishes at every finite object $X$.

We name some more objects of $\mathcal{T}_{Y}$ : If $Z$ is a space and $Z \stackrel{f}{\rightarrow} Y$ is a map then let $Y+_{f} Z$ be the disjoint union of $\mathrm{Y}$ and $\mathrm{Z}$, considered as an object of $\mathcal{T}_{Y}$ in the evident way. 
If $X$ is an object of $\mathcal{T}_{Y}$ obtained by attaching an $m$-cell to another object $X^{\prime}$, then there is a cocartesian diagram

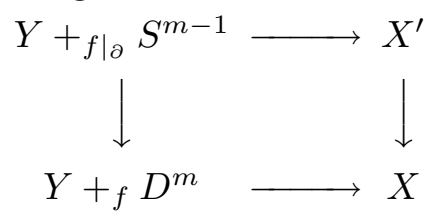

for some $D^{m} \stackrel{f}{\rightarrow} Y$, and thus a cartesian diagram

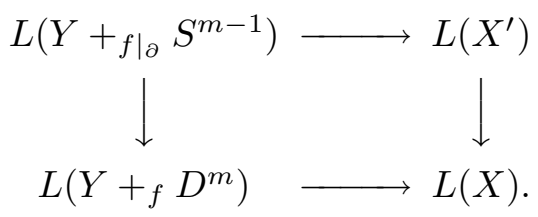

$L(X)$ will be contractible if the other three spectra are. Thus an induction on the number of cells in $X-i(Y)$ will be possible as soon as we have dealt with the cases $X=Y+{ }_{f} D^{m}$ and $X=Y+{ }_{f} S^{m}$. The sphere case follows from the disk case by induction on $m$ (beginning with the case $Y+{ }_{f} S^{-1}=Y$ ), and the disk case is taken care of by a weak equivalence

$$
Y \vee_{y} S^{0} \rightarrow Y+{ }_{f} D^{n}
$$

obtained by choosing a point $y \in f\left(D^{n}\right)$.

Note that if $\mathrm{Y}$ is path-connected then it is only necessary to verify the hypothesis of 5.8 for one choice of $\left(y_{1}, \ldots, y_{n}\right)$, since a path $I \stackrel{f}{\rightarrow} Y$ from $y$ to $y^{\prime}$ in $Y$ yields a diagram of equivalences in $\mathcal{T}_{Y}$ :

$$
Y \vee_{y} S^{0} \stackrel{\sim}{\longrightarrow} Y+_{f} I \stackrel{\sim}{\longleftarrow} Y \vee_{y^{\prime}} S^{0} .
$$

There is a variant of 5.8, with the same proof, for unreduced-multilinear functors from $\mathcal{U}_{Y}$ to spectra, with a point over $y_{j}$ replacing $Y \vee_{y_{j}} S^{0}$. The proof of 5.8 also generalizes rather easily to prove that a map between $d$-excisive homotopy functors from $\mathcal{T}_{Y}$ [resp. $\mathcal{U}_{Y}$ ] to $\mathcal{S} p$ must be an equivalence for all objects if it is an equivalence for all objects of the form $Y+{ }_{f} S$ [resp. $S$ ] where $S$ is a discrete set of at most $d$ points with a map to $X$.

For us the main consequence of 5.8 is:

Corollary 5.9 If a map $F \rightarrow G$ of homotopy functors $\mathcal{U} \rightarrow \mathcal{T}$ induces an equivalence of $n^{\text {th }}$ derivatives

$$
\partial_{y_{1}, \ldots, y_{n}} F(Y) \rightarrow \partial_{y_{1}, \ldots, y_{n}} G(Y)
$$

for every point $\left(y_{1}, \ldots, y_{n}\right) \in Y^{n}$ then it induces an equivalence of $n^{\text {th }}$ differentials

$$
D_{n} F_{Y}(X) \rightarrow D_{n} G_{Y}(X)
$$

for every finite object $X$ of $\mathcal{U}_{Y}$ or $\mathcal{T}_{Y}$. 
Proof Apply 5.8 to $D^{(n)} F_{Y} \rightarrow D^{(n)} G_{Y}$, noting that the behavior of $D_{n} F_{Y}$ on finite objects is determined by the behavior of $D^{(n)} F_{Y}$ on finite objects.

These statements definitely require spectra rather than spaces as the output of the functors. For example, if $C$ is a spectrum and $L$ is the linear functor from based spaces to based spaces given by

$$
L(X)=\Omega^{\infty}(C \wedge X),
$$

then the statement that $L\left(S^{0}\right)$ is contractible means only that the homotopy groups $\pi_{j}(C)$ are trivial for $j \geq 0$.

The restriction to finite objects in the results above can be removed if $F$ satisfies a suitable limit axiom.

5.10 Definition A homotopy functor $\mathcal{C} \stackrel{F}{\longrightarrow} \mathcal{D}$ is finitary if it preserves filtered homotopy colimits up to weak equivalence, that is, if the natural map

$$
\underset{\alpha}{\operatorname{hocolim}} F\left(X_{\alpha}\right) \rightarrow F\left(\underset{\alpha}{\operatorname{hocolim}} X_{\alpha}\right)
$$

is a weak equivalence whenever $\left\{X_{\alpha}\right\}$ is a diagram in $\mathcal{C}$ indexed by a filtering category.

In the case of linear functors from spaces to spaces, this condition means that the corresponding homology theory satisfies Milnor's wedge axiom. It is clear that the $n^{\text {th }}$ Taylor approximation of a finitary functor is itself finitary.

5.11 Remark This was called the limit axiom in [10], but it seems useful to have an adjective available. The term "continuous functor" has been used, but we would rather reserve that for something else (a functor that behaves continuously on morphisms).

Since every space is equivalent to a filtered hocolim of finite CW complexes, a finitary functor of spaces is determined (up to natural weak equivalence) by its behavior on finite complexes. More generally a finitary functor of objects in $\mathcal{C}_{Y}$ is determined by its behavior on objects that are finite in the sense of 5.7.

According to 5.9 the $n^{\text {th }}$ differential (which knows all about the $n^{\text {th }}$ layer of the Taylor tower) is in some sense controlled by the $n^{\text {th }}$ derivative. This is only a weak sense, however, since 5.9 cannot produce an equivalence between the differentials of $F$ and $G$ unless a map between $F$ and $G$ is already given. It would be better to have a way of building the $n^{\text {th }}$ differential from the $n^{\text {th }}$ derivative.

The $n^{\text {th }}$ derivative of $F$ at $Y$ gives a spectrum for each ordered $n$-tuple of points in $Y$. To identify the $n^{\text {th }}$ differential, one needs just a little more information. We will sketch one of several possible answers to the following vague question: 
5.13 Vague question How can $D^{(n)} F$ be assembled from the spectra $\partial_{y_{1}, \ldots, y_{n}}^{(n)} F(Y)$ ?

Answers in the case $n=1$ tend to generalize easily to the general case, so we will concentrate on that case.

A useful point of view is that a (finitary) linear functor of spaces over $Y$ corresponds to a "coefficient system" on $Y$ which assigns a spectrum $E_{y}$ to each point $y$. The spectrum $E_{y}$ must depend on the point $y$ continuously in some sense, so the linear functor cannot really be specified by merely giving $E_{y}$ for each $y$. (That would be like trying to specify a vector bundle by giving all of its fibers, a mere collection of vector spaces.)

One can give rigorous sense to this idea by making the following definition: A system of spectra on $Y$ consists of objects $\left\{E_{n}\right\}$ in $\mathcal{T}_{Y}$ such that the structural maps $E_{n} \rightarrow Y$ are fibrations, related by maps

$$
\Sigma_{Y} E_{n} \rightarrow E_{n+1} \text {. }
$$

We may write $E_{y, n}$ for the fiber of $E_{n} \rightarrow Y$ over $y \in Y$. The spaces $\left\{E_{y, n}\right\}$ for fixed $y$ constitute a spectrum $E_{y}$, the fiber of $E$ over $y$.

Such a system $E$ determines a prespectrum whose $n^{\text {th }}$ space is the homotopy cofiber of the structural map $Y \rightarrow E_{n}$. The associated spectrum, which may be thought of as the homology of $Y$ with coefficients in $E$, will be denoted by

$$
\int_{y \in Y} E_{y} d y
$$

Of course this notation is deceptive, since it appears not to matter how the various spectra $E_{y}$ are related.

The integral signs are not meant to suggest antidifferentiation.

We draw attention to the familiar special case when $E$ is a "trivial bundle of spectra" over $Y$ : Let $C$ be a spectrum and take $E_{n}$ to be $Y \times C_{n}$. Then

$$
\int_{y \in Y} E_{y} d y=\int_{y \in Y} C d y \cong C \wedge Y .
$$

In the general case there is a spectral sequence of Atiyah-Hirzebruch type, with

$$
E_{p, q}^{2} H_{p}\left(Y ; \pi_{q}\left(E_{y}\right)\right) \Rightarrow \pi_{p+q} \int_{y \in Y} E_{y} d y .
$$

One constructs it by taking the direct limit over $n$ of a spectral sequence with

$$
E_{p, q}^{2}=H_{p}\left(Y ; \pi_{q+n}^{S}\left(E_{n, y}\right)\right) \Rightarrow \pi_{p+q+n}^{S} \operatorname{hocofiber}\left(Y \rightarrow E_{n}\right) .
$$


Systems of spectra pull back: a map $X \stackrel{f}{\rightarrow} Y$ and a system $E$ on $Y$ determine a system $f^{*} E$ on $X$ given by $\left(f^{*} E\right)_{n}=E_{n} \times_{Y} X$, whose fiber at $x \in X$ is isomorphic to $E_{f(x)}$. Thus a system $E$ on $Y$ gives a functor from $\mathcal{U}_{Y}$ to $\mathcal{S} p$ :

$$
X=(X, X \stackrel{f}{\rightarrow} Y) \mapsto \int_{x \in X} E_{f(x)} d x .
$$

This is a homotopy functor, and it is finitary and 1-excisive; all of these assertions can proved by spectral sequence comparison arguments. This functor vanishes at the empty set, so it is not linear but rather unreduced-linear (4.5). Reducing it, one gets a linear functor, which sends $X$ to the homotopy fiber of

$$
\int_{x \in X} E_{f(x)} d x \rightarrow \int_{y \in Y} E_{y} d y .
$$

It is fairly clear that one could classify the finitary linear functors on $\mathcal{T}_{Y}$ along these lines, but we will not pursue that here. The generalization to $n>1$ involves systems of spectra over $Y^{n}$ with a $\Sigma_{n}$-symmetry, or alternatively systems of spectra over $Y^{n} \times_{\Sigma_{n}} E \Sigma_{n}$.

If $Y$ is equivalent to the classifying space of a group $G$ then systems of spectra on $Y$ are the same (at the homotopy category level) as spectra with $G$-action. This idea can be extended to simplicial groups $G$, so that it applies to all based connected $Y$.

The idea of constructing linear functors by systems of spectra over a space was implicitly present in sections 2 and 3 of [9]. The systems that arose there were mainly "fiberwise suspension spectra" in the following sense: If $W$ is an object of $\mathcal{T}_{Y}$ whose structural map $W \rightarrow Y$ is a fibration, then the repeated fiberwise suspensions $\Sigma_{Y}^{n} W$ form a system $E$ whose fiber $E_{y}$ is the suspension spectrum of the fiber of $W$ over $y$.

There is also a dual construction, in which $E$ is used as coefficients for twisted cohomology rather than twisted homology. If $E$ is a system of spectra on a CW complex $K$ then the spectrum

$$
\int^{k \in K} E_{k} d k
$$

is defined by letting the $n^{\text {th }}$ space be the space of sections of the fibration $E_{n} \rightarrow Y$. If $K$ is locally compact then there is also the compactly supported version

$$
\int_{c}^{k \in K} E_{k} d k
$$

made out of spaces of compactly supported sections. 
If $X \mapsto E(X)$ is a homotopy functor from spaces to systems of spectra on the finite complex $K$ then "differentiation under the integral" is valid; there is an equivalence

$$
\partial_{y} \int^{k \in K} E_{k}(Y) d k \sim \int^{k \in K} \partial_{y} E_{k}(Y) d k .
$$

The proof is by induction on the number of cells in $K$. This extends to the compactly supported case if for example the one-point compactification of $K$ is a finite complex.

We will also need the following simple principle: If $P \stackrel{\pi}{\rightarrow} B$ is a principal $G$-bundle ( $G$ being a topological group) and $E$ is a system of spectra on the locally compact space $B$, then when $G$ is made to act on the spectrum

$$
\int^{p \in P} E_{\pi(p)} d p
$$

in the obvious way the homotopy fixed point spectrum is equivalent to

$$
\int^{b \in B} E_{b} d b
$$

Confession We should really distinguish between (fiberwise) unreduced suspension and (fiberwise) reduced suspension. The former, (fiberwise) join with a two-point set, is what we ordinarily denote by $\Sigma_{Y}$ here, and it has the pleasant feature that it takes fibrations to fibrations. The latter, on the other hand, is much better for making spectra. When they are inequivalent, we have to choose the former, but this must be paid for by growing whiskers. We have not systematically imposed a solution of this small technical difficulty on the reader, because we do not have a neat solution.

\section{Multilinearized cross-effects}

Before working on some examples, we need one more tool.

6.1 Theorem Let $\mathcal{D}$ be $\mathcal{T}$ or $\mathcal{S} p$. The $n^{\text {th }}$ differential $D^{(n)} F=c r_{n} D_{n} F$ of a homotopy functor $\mathcal{T}_{Y} \stackrel{F}{\longrightarrow} \mathcal{D}$ is (naturally, weakly) equivalent to the multilinearization of the $n^{\text {th }}$ cross-effect $c r_{n} F$ of $F$ itself. 
This means that $\left(D^{(n)} F\right)\left(X_{1}, \ldots, X_{n}\right)$ is essentially the homotopy colimit of

$$
\Omega^{i_{1}+\cdots+i_{n}}\left(c r_{n} F\right)\left(\Sigma_{Y}^{i_{1}} X_{1}, \ldots, \Sigma_{Y}^{i_{n}} X_{n}\right)
$$

over $\left(i_{1}, \ldots, i_{n}\right)$. The latter may also be described as

$$
\underset{i}{\operatorname{hocolim}} \Omega^{i V_{n}}\left(c r_{n} F\right)\left(\Sigma_{Y}^{i} X_{1}, \ldots, \Sigma_{Y}^{i} X_{n}\right) \text {. }
$$

In particular the $n^{\text {th }}$ derivative $\partial_{y_{1}, \ldots, y_{n}}^{(n)} F(Y)$ is equivalent to the associated spectrum of a prespectrum whose $i^{\text {th }}$ space is

$$
\Omega^{i \bar{V}_{n}}\left(c r_{n} F\right)\left(Y \vee_{y_{1}} S^{i}, \ldots, Y \vee_{y_{n}} S^{i}\right) .
$$

6.1 can be a key tool for identifying $D_{n} F$ in examples. While the definitions of $P_{n} F$ and $D_{n} F$ are difficult to use for explicit calculation when $n$ is greater than one, we do know how to recover $D_{n} F$ from $c r_{n} D_{n} F$, and 6.1 says that this in turn can be obtained rather directly from $F$ itself.

6.2 Remark Like 1.7 and $2.1,6.1$ has an easier proof in the case when the functor $F$ is analytic. In fact, in that case using 1.5(2) one sees easily that if the maps $X_{j} \rightarrow Y$ are all $k$-connected then the map

$$
\left(c r_{n} F\right)\left(X_{1}, \ldots, X_{n}\right) \rightarrow\left(c r_{n} P_{n} F\right)\left(X_{1}, \ldots, X_{n}\right)
$$

induced by $p_{n} F$ is $\left((n+1) k-c^{\prime}\right)$-connected for some constant $c^{\prime}$, from which it follows that the canonical map from

$$
\Omega^{i V_{n}}\left(c r_{n} F\right)\left(\Sigma^{i} X_{1}, \ldots, \Sigma^{i} X_{n}\right)
$$

to

$$
\Omega^{i V_{n}}\left(c r_{n} P_{n} F\right)\left(\Sigma^{i} X_{1}, \ldots, \Sigma^{i} X_{n}\right) \sim\left(c r_{n} P_{n} F\right)\left(X_{1}, \ldots, X_{n}\right)
$$

has a connectivity tending to $\infty$ with $i$. For that matter, in applying 6.1 to a particular $F$ one often uses this same kind of reasoning again: One identifies the multilinearization of $c r_{n} F$ with a given functor $L$ by exhibiting a (natural and symmetry-preserving) map

$$
\left(c r_{n} F\right)\left(X_{1}, \ldots, X_{n}\right) \rightarrow L\left(X_{1}, \ldots, X_{n}\right)
$$

and checking that it is $\left((n+1) k-c^{\prime}\right)$-connected for some constant $c^{\prime}$ when the $X_{j} \rightarrow Y$ are $k$-connected. Therefore some readers may prefer to skip to Section 7 after the proof of 6.3 .

A consequence of 6.1 is that what we are calling a second derivative (that is, the coefficient of the bilinear functor corresponding to a 2-homogeneous layer) can actually be seen as the derivative of a derivative. More generally, we have the following useful interpretation of 6.1 : 


\subsection{Corollary}

$$
\partial_{y_{1}, \ldots, y_{p+q}}^{(p+q)} F(Y) \sim \partial_{y_{1}, \ldots, y_{p}}^{(p)} \partial_{y_{p+1}, \ldots, y_{p+q}}^{(q)} F(Y)
$$

Explanation and sketch of proof If $F$ is a homotopy functor from spaces to either based spaces or spectra, then, as we have already observed, the spectrum $\partial_{y_{p+1, \ldots, y_{p}+q}}^{(q)} F(Y)$ depends functorially on $Y=\left(Y, y_{p+1} \ldots, y_{p+q}\right)$ with appropriate definitions. In stating 6.3 we are extending the "partial derivative" notation to cover functors of "spaces with several base points". Thus, for example, if $F$ is a functor of based spaces then $\partial_{y} F\left(Y, y_{0}\right)$ depends functorially on $\left(Y, y, y_{0}\right)$ and is defined as the coefficient spectrum of the linear part of the functor

$$
Z \mapsto F\left(Y \vee_{y} Z\right)
$$

Here $y_{0} \in Y$ is serving as basepoint in $Y \vee_{y} Z$ for purposes of applying the functor $F$ while $y$ is serving as basepoint in $Y$ for wedging with $Z$.

In view of 6.1 , to prove 6.3 one has only to see that for a functor of $p+q$ based spaces, such as

$$
\left(Z_{1}, \ldots, Z_{p+q}\right) \mapsto F\left(Y \vee_{y_{1}} Z_{1}, \ldots, Y \vee_{y_{n}} Z_{p+q}\right),
$$

the following two processes are equivalent: (1) reducing in all variables followed by multilinearizing in all variables, (2) reducing and multilinearizing in the last $q$ variables, followed by reducing and multilinearizing in the first $p$ variables. This is easy.

Another consequence of 6.1 is:

6.4 Corollary $F$ is $m$-reduced if and only if $F$ is reduced and for every $0<n<m$ the multilinearization of the $n^{\text {th }}$ cross-effect of $F$ is contractible.

Proof This is clear from 6.1 and the fact that $c r_{n} D_{n} F$ determines $D_{n} F$.

The proof of 6.1 is connected with the idea of "multivariable Taylor series" (Remark 1.22). A key observation is that the product category $\mathcal{C}_{Y_{1}} \times \cdots \times \mathcal{C}_{Y_{n}}$ is itself the category of spaces over a space: it is equivalent to $\mathcal{C}_{Y_{1}} \amalg \ldots \amalg Y_{n}$ where the "Ш" denotes disjoint union. In this equivalence of categories, $n$-tuples of weak equivalences correspond to weak equivalences and $n$-tuples of cocartesian cubes correspond to cocartesian cubes. When it is necessary to distinguish between a functor

$$
\mathcal{C}_{Y_{1}} \times \cdots \times \mathcal{C}_{Y_{n}} \stackrel{F}{\longrightarrow} \mathcal{D}
$$


of $n$ variables and the associated functor

$$
\mathcal{C}_{Y_{1} \amalg \ldots \amalg Y_{n}} \rightarrow \mathcal{D}
$$

of one variable, we will denote the latter by $\lambda F$.

6.1 is proved using the following statement, which will be proved at the end of Section 6:

6.5 Lemma If $\mathcal{C}_{Y_{1}} \times \cdots \times \mathcal{C}_{Y_{n}} \stackrel{G}{\longrightarrow} \mathcal{D}$ is $(1, \ldots, 1)$-reduced then $\lambda P_{1, \ldots, 1} G \sim$ $P_{n} \lambda G$.

Proof of 6.1 Applying 6.5 in the special case $Y=Y_{1}=\cdots=Y_{n}$, with $G=c r_{n} F$, we find that what we need is a natural equivalence

$$
P_{n} \lambda c r_{n} F \sim \lambda c r_{n} P_{n} F .
$$

This will follow from a natural equivalence $T_{n} \lambda c r_{n} F \sim \lambda c r_{n} T_{n} F$, which in turn will follow from a natural equivalence $J_{U} \lambda c r_{n} F \sim \lambda c r_{n} J_{U} F$, where (as in the proof of 1.8) $J_{U}$ is composition with the fiberwise join with a finite set $U$. $\lambda \circ \mathrm{Cr}_{n}$ is a three-step process: Compose with the (whiskered) sum

$$
\mathcal{T}_{Y}^{n} \stackrel{+}{\longrightarrow} \mathcal{T}_{Y},
$$

then reduce in all variables (this was called $r$ in the proof of 4.2), then compose with the equivalence of categories

$$
\mathcal{T}_{Y} \amalg \cdots \amalg Y \rightarrow \mathcal{T}_{Y}^{n} .
$$

Each of these steps commutes with $J_{U}$ up to natural equivalence.

To get to 6.5 we must revisit and strengthen some results that were discussed in Section 3. The following is a strengthening of $[10 ; 3.4]$.

6.6 Lemma If a homotopy functor $\mathcal{C}_{Y_{1}} \times \cdots \times \mathcal{C}_{Y_{n}} \stackrel{F}{\rightarrow} \mathcal{D}$ is $\left(d_{1}, \ldots, d_{n}\right)$-excisive then $\lambda F$ is $\left(d_{1}+\cdots+d_{n}\right)$-excisive.

Proof In fact the proof of $[10 ; 3.4]$ becomes a proof of 6.6 if one uses $\left(X_{1}, \ldots\right.$, $\left.X_{n}\right)$ in place of $(X, \ldots, X)$ throughout.

In particular $\lambda F$ is $n$-excisive if $F$ is $(1, \ldots, 1)$-excisive. We need to know also that $\lambda F$ is $n$-homogeneous if $F$ is multilinear. The following is a strengthening of 3.1 . 
6.7 Lemma If $\mathcal{C}_{Y_{1}} \times \cdots \times \mathcal{C}_{Y_{n}} \stackrel{F}{\longrightarrow} \mathcal{D}$ is $(1, \ldots, 1)$-reduced, then $\lambda F$ is $n$ reduced.

Proof This follows from the next statement as 3.1 followed from 3.2.

6.8 Lemma If $\mathcal{C}_{Y_{1}} \times \cdots \times \mathcal{C}_{Y_{n}} \stackrel{F}{\longrightarrow} \mathcal{D}$ is $(1, \ldots, 1)$-reduced, then the map

$$
\lambda F \stackrel{t_{n-1} \lambda F}{\longrightarrow} T_{n-1} \lambda F
$$

factors through a weakly contractible functor.

Proof Again, the proof of 3.2 applies with no change except $\left(X_{1}, \ldots, X_{n}\right)$ for $(X, \ldots, X)$.

We need this partial converse to 6.6 :

6.9 Lemma If $\mathcal{C}_{Y_{1}} \times \cdots \times \mathcal{C}_{Y_{n}} \stackrel{L}{\longrightarrow} \mathcal{D}$ is $(1, \ldots, 1)$-reduced and $\lambda L$ is $n$-excisive, then $L$ is multilinear.

Proof We have to show that $L$ is 1 -excisive in each variable. It is sufficient to consider the last variable. Fix an object $X_{j}$ of $\mathcal{C}_{Y_{j}}$ for each $1 \leq j \leq n-1$ and let

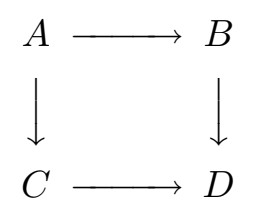

be any cocartesian square in $\mathcal{C}_{Y_{n}}$. We have to show that the square

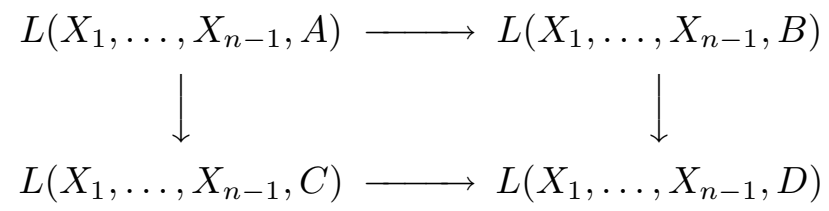

is cartesian.

Define an $(n+1)$-cube in $\mathcal{C}_{Y_{1}} \times \cdots \times \mathcal{C}_{Y_{n}}$ as follows: For each subset $S$ of $\{1, \ldots, n-1\}$, let $X_{j}(S)$ be $Y_{j}$ if $j \in S$ and $X_{j}$ if $j \notin S$.

Then $\left\{\left(X_{1}(S), \ldots, X_{n-1}(S)\right)\right\}$ constitutes an $(n-1)$-cube in $\mathcal{C}_{Y_{1}} \times \cdots \times \mathcal{C}_{Y_{n-1}}$ and our $(n+1)$-cube will consist of the squares

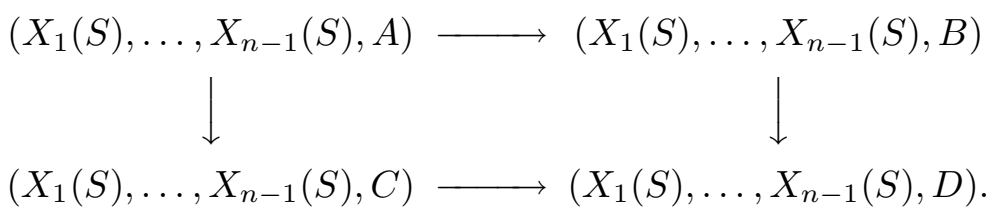

Geometry $\&$ Topology, Volume 7 (2003) 
The $(n+1)$-cube is strongly cocartesian, so $L$ yields a cartesian cube. On the other hand, for each nonempty $S$ the square

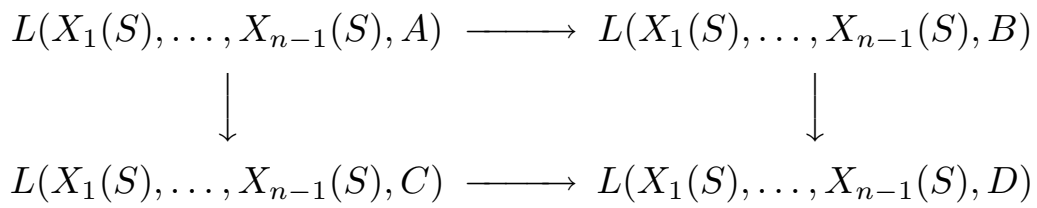

is cartesian; in fact it is made up of contractible objects because $L$ is reduced in each variable. It follows by 1.6 of [10] that the square corresponding to $S=\emptyset$ is also cartesian.

Proof of 6.5 Now it is convenient to drop the distinction between $F$ and $\lambda F . P_{1, \ldots, 1} F$ is the universal example of a $(1, \ldots, 1)$-excisive functor under $F$, and is also $n$-excisive (by 6.6). $P_{n} F$ is the universal example of an $n$-excisive functor under $F$, and is also $(1, \ldots, 1)$-excisive (by 6.9$)$. It follows that they are the same.

The reader, looking at 6.6 and 6.7 , might have wondered about:

6.10 Lemma If a homotopy functor $\mathcal{C}_{Y_{1}} \times \cdots \times \mathcal{C}_{Y_{n}} \stackrel{F}{\longrightarrow} \mathcal{D}$ is $\left(d_{1}, \ldots, d_{n}\right)$ reduced then $\lambda F$ is $\left(d_{1}+\cdots+d_{n}\right)$-reduced.

In fact this is true, and it can be deduced from 6.4.

\section{$7 \quad$ Example: Suspension spectra of mapping spaces}

For an unbased space $X$ let $\Sigma_{+}^{\infty} X$ be the suspension spectrum of the based space $X_{+}$obtained by adding an extra point to $X$. We will call this the unreduced suspension spectrum (and hope that this does not lead anyone to confuse the unreduced suspension of $X$ with $S^{1} \wedge X_{+}$). Similarly, if $X$ is a space fibered over $Y$ we can speak of its unreduced fiberwise suspension spectrum, meaning the fiberwise suspension spectrum of the fiberwise based space $\psi X$, where as in Section 4 this means the disjoint union of $X$ and $Y$ considered as an object of $\mathcal{T}_{Y}$.

For a finite CW complex $K$, the functor $F(X)=\Sigma_{+}^{\infty} X^{K}$ is analytic by [10; 4.4], and its first derivative was found in [9;2.4]. We now find its $n^{\text {th }}$ derivative. We begin by recalling what the formula for the first derivative is and where that formula came from. 
In the notation of Section 5 the formula for the first derivative is

$$
\partial_{y} \Sigma_{+}^{\infty} Y^{K} \sim \int^{k \in K} \Sigma_{+}^{\infty}(Y, y)^{(K, k)} d k
$$

Recall that this means that the linearization of the functor

$$
\begin{aligned}
Z & \mapsto \operatorname{hofiber}\left(\Sigma_{+}^{\infty}\left(Y \vee_{y} Z\right)^{K} \rightarrow \Sigma_{+}^{\infty} Y^{K}\right) \\
& \sim \Sigma^{\infty} \operatorname{hocofiber}\left(Y^{K} \rightarrow\left(Y \vee_{y} Z\right)^{K}\right)
\end{aligned}
$$

is the functor

$$
Z \mapsto Z \wedge \int^{k \in K} \Sigma_{+}^{\infty}(Y, y)^{(K, k)} d k
$$

Because $K$ is finite, this last can also be written

$$
\int^{k \in K} \Sigma^{\infty}\left(Z \wedge(Y, y)^{(K, k)}\right) d k .
$$

Implicitly in 7.1 we are using a certain system of spectra on $K$, namely the unreduced fiberwise suspension spectrum of a certain space over $K$, let us call it $W$, whose fiber over $k \in K$ is $W_{k}=(Y, y)^{(K, k)} ; W$ is the subspace of $Y^{K} \times K$ consisting of pairs $(f, k)$ such that $f(k)=y$. Likewise in 7.3 we are using the fiberwise suspension spectrum of a space over $K$, call it $Z \wedge_{K} \psi W$, whose fiber is $Z \wedge W_{k+}$, namely

$$
\operatorname{colim}(Z \times W \leftarrow W \rightarrow K)
$$

7.4 Remark A formula for the differential and not just the derivative was given in [9]. In the present notation it says that the unreduced-linear functor corresponding to $D_{Y} F$ takes the object $X \stackrel{f}{\rightarrow} Y$ to

$$
\int_{x \in X} \int^{k \in K} \Sigma_{+}^{\infty}(Y, f(x))^{(K, k)} d k d x
$$

In other words, the linear functor is given by a coefficient system on $Y$ which may be obtained by "integration over the fiber" from a system on $K \times Y$, the fiberwise unreduced suspension spectrum of the fibration $K \times Y^{K} \rightarrow K \times Y$ whose fiber over $(k, y)$ may be identified with $(Y, y)^{(K, k)}$.

The method of proof for 7.1 was this: First give a natural map from 7.2 to 7.3, then show that its connectivity is roughly twice that of the space $Z$.

To produce the map, it was enough to give a natural map of based spaces

$$
\operatorname{hocofiber}\left(Y^{K} \rightarrow\left(Y \vee_{y} Z\right)^{K}\right) \rightarrow \Omega^{\infty} \int^{k \in K} \Sigma^{\infty}\left(Z \wedge(Y, y)^{(K, k)}{ }_{+}\right) d k \text {. }
$$


This was done by means of a tautological map from $\left(Y \vee_{y} Z\right)^{K}$ to the space of sections of $Z \wedge_{K} \psi W \rightarrow K$. To specify this map we say where it sends the map $K \stackrel{\tilde{f}}{\rightarrow} Y \vee_{y} Z$. Let $f$ be the composed map

$$
K \stackrel{\tilde{f}}{\rightarrow} Y \vee_{y} Z \rightarrow Y
$$

Then $\tilde{f}$ is sent to the section whose value at $k$ is $\tilde{f}(k) \wedge f \in Z \wedge(Y, y)^{(K, k)}+$ if $\tilde{f}(k) \in Z$ and otherwise is the (fiberwise) basepoint.

It is a good precaution to add a "whisker" to $Z$, replacing $Y \vee_{y} Z$ by $Y \cup_{y}$ $I \cup_{z} Z$, before making the construction just described, to insure that the map $Z \wedge_{Y} \psi W \rightarrow K$ is a fibration.

The proof that the resulting map from 7.2 to 7.3 is highly connected will not be repeated here.

There is a variant of 7.1 for spaces of based maps: If $K$ has a basepoint $k_{0}$, then we obtain

$$
\partial_{y} \Sigma_{+}^{\infty}\left(Y, y_{0}\right)^{\left(K, k_{0}\right)} \sim \int_{c}^{k \in K-\left\{k_{0}\right\}} \Sigma_{+}^{\infty}\left(Y, y, y_{0}\right)^{\left(K, k, k_{0}\right)} d k
$$

(This time the whisker is even more important, because it insures that even if $y=y_{0}$ the section being constructed will have compact support.)

Now we are in a position to compute a second derivative, using 6.2. We have:

$$
\begin{aligned}
\partial_{y_{1}} \partial_{y_{2}} \Sigma_{+}^{\infty} Y^{K} & \sim \partial_{y_{1}} \int^{k_{2} \in K} \Sigma_{+}^{\infty}\left(Y, y_{2}\right)^{\left(K, k_{2}\right)} d k_{2} \\
& \sim \int^{k_{2} \in K} \partial_{y_{1}} \Sigma_{+}^{\infty}\left(Y, y_{2}\right)^{\left(K, k_{2}\right)} d k_{2} \\
& \sim \int^{k_{2} \in K} \int_{c}^{k_{1} \in K-\left\{k_{2}\right\}} \Sigma_{+}^{\infty}\left(Y, y_{1}, y_{2}\right)^{\left(K, k_{1}, k_{2}\right)} d k_{1} d k_{2} \\
& \sim \int_{c}^{\left(k_{1}, k_{2}\right) \in K^{(2)}} \Sigma_{+}^{\infty}\left(Y, y_{1}, y_{2}\right)^{\left(K, k_{1}, k_{2}\right)} d\left(k_{1}, k_{2}\right) .
\end{aligned}
$$

The differentiation under the integral sign depends on the hypothesis that $K$ is finite. $K^{(2)}$ is the complement of the diagonal in $K \times K$. The last step, replacing a double integral by a single integral, is a tautology.

The expression in the last line implicitly refers to a system of spectra on $K^{(2)}$ whose fiber at $\left(k_{1}, k_{2}\right)$ is $\Sigma_{+}^{\infty}\left(Y, y_{1}, y_{2}\right)^{\left(K, k_{1}, k_{2}\right)}$, namely the unreduced fiberwise suspension spectrum of a certain fibration

$$
W^{[2]} \rightarrow K^{(2)}
$$


The space $W^{[2]}$ is the subspace of $Y^{K} \times K^{(2)}$ consisting of all $\left(f, k_{1}, k_{2}\right)$ such that $f\left(k_{1}\right)=y_{1}$ and $f\left(k_{2}\right)=y_{2}$, so that the fiber $W_{\left(k_{1}, k_{2}\right)}^{[2]}$ over $\left(k_{1}, k_{2}\right)$ is $\left(Y, y_{1}, y_{2}\right)^{\left(K, k_{1}, k_{2}\right)}$.

This is not enough to determine the quadratic functor $D_{2} F_{Y}$, because we do not yet know the $\Sigma_{2}$-symmetry in the second derivative. We can make a good guess about that, because the last expression in 7.5 has an obvious symmetry. To verify the guess, we can proceed as follows:

We have to study the second-order cross-effect of $F_{Y}$ as applied to objects $Y \vee_{y} Z$, in other words the total homotopy fiber of

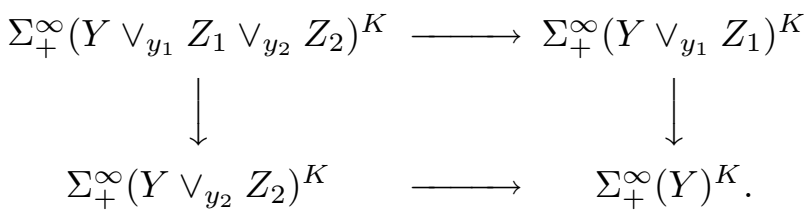

We know that the bilinearization of this is equivalent to

$$
Z_{1} \wedge Z_{2} \wedge \int_{c}^{\left(k_{1}, k_{2}\right) \in K^{(2)}} \Sigma_{+}^{\infty}\left(Y, y_{1}, y_{2}\right)^{\left(K, k_{1}, k_{2}\right)} d\left(k_{1}, k_{2}\right),
$$

or equivalently

$$
\int_{c}^{\left(k_{1}, k_{2}\right) \in K^{2}-\Delta} \Sigma^{\infty}\left(Z_{1} \wedge Z_{2} \wedge\left(Y, y_{1}, y_{2}\right)^{\left(K, k_{1}, k_{2}\right)}{ }_{+}\right) d\left(k_{1}, k_{2}\right) .
$$

This last expression refers to the fiberwise suspension spectrum of a certain space over $K^{(2)}$ whose fibers are $Z_{1} \wedge Z_{2} \wedge W_{\left(k_{1}, k_{2}\right)_{+}}^{[2]}$. Call it $\left(Z_{1} \wedge Z_{2}\right) \wedge_{K^{(2)}}$ $\psi W^{[2]}$.

We are seeking to show that that equivalence can be chosen to preserve the $\Sigma_{2}$-symmetry, so we should look for a symmetry-preserving map from the total homotopy fiber of 7.6 to 7.7 .

The total homotopy fiber of 7.6 can be rewritten as the suspension spectrum of the total cofiber of

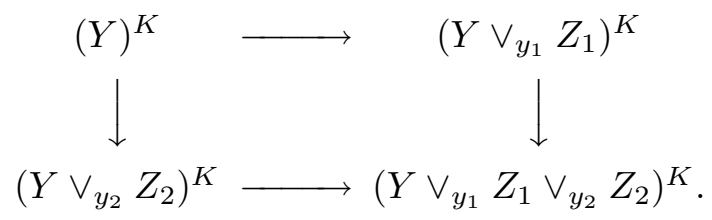

There is a tautological map from this total cofiber to the zeroth space of 7.7. It arises from a tautological map from $\left(Y \vee_{y_{1}} Z_{1} \vee_{y_{2}} Z_{2}\right)^{K}$ to the space of 
compactly supported sections of $\left(Z_{1} \wedge Z_{2}\right) \wedge_{Y} W^{[2]} \rightarrow K^{(2)}$. To specify this we say where it sends the map $K \stackrel{\tilde{f}}{\rightarrow} Y \vee_{y_{1}} Z_{1} \vee_{y_{2}} Z_{2}$. Let $f$ be the composed map

$$
K \stackrel{\tilde{f}}{\rightarrow} Y \vee_{y_{1}} Z_{1} \vee_{y_{2}} Z_{2} \rightarrow Y
$$

Then $\tilde{f}$ is sent to the section whose value at $\left(k_{1}, k_{2}\right)$ is $\tilde{f}\left(k_{1}\right) \wedge \tilde{f}\left(k_{2}\right) \wedge\left(f, k_{1}, k_{2}\right)$ if $\tilde{f}\left(k_{1}\right) \in Z_{1}$ and $\tilde{f}\left(k_{2}\right) \in Z_{2}$ and otherwise the (fiberwise) basepoint.

We claim, leaving the remaining details to the reader, that this results in a map

$$
\partial_{y_{1}, y_{2}}^{(2)} \Sigma^{\infty}\left(Y_{+}^{K}\right) \rightarrow \int_{c}^{\left(k_{1}, k_{2}\right) \in K^{(2)}} \Sigma_{+}^{\infty}\left(Y, y_{1}, y_{2}\right)^{\left(K, k_{1}, k_{2}\right)} d\left(k_{1}, k_{2}\right)
$$

that corresponds to 7.5 under 6.2 and is therefore an equivalence.

The same method gives the $n^{\text {th }}$ derivative. The conclusion is:

7.10 Theorem For a finite complex $K$ we have a symmetry-preserving equivalence

$$
\partial_{y_{1}, \ldots y_{n}}^{(n)} \Sigma_{+}^{\infty} Y^{K} \sim \int_{c}^{k \in K^{(n)}} \Sigma_{+}^{\infty}\left(Y, y_{1}, \ldots, y_{n}\right)^{\left(K, k_{1}, \ldots, k_{n}\right)} d k
$$

Here $K^{(n)}$ is the space of all ordered $n$-tuples $k=\left(k_{1}, \ldots, k_{n}\right)$ of distinct points in $K$.

It is interesting to work out what this says in the case when $K$ is a finite set of cardinality $m$, and to compare it with the formula

$$
m(m-1) \ldots(m-n-1) y^{m-n} .
$$

for the $n^{\text {th }}$ derivative of $y^{m}$ in ordinary calculus.

In the case when $Y$ is a one-point space, the right-hand side of 7.10 becomes

$$
\int_{c}^{k \in K^{(n)}} \Sigma^{\infty} S^{0} d k \sim \operatorname{Map}_{*}\left(K^{(n) c}, \Sigma^{\infty} S^{0}\right)=\left(K^{(n) c}\right)^{*}
$$

In other words, the $n^{\text {th }}$ derivative of $F(X)=\Sigma_{+}^{\infty} X^{K}$ at a point is the $S$-dual $\left(K^{(n) c}\right)^{*}$ of a certain based $\Sigma_{n}$-space $K^{(n) c}$, the one-point compactification of $K^{(n)}$ (or the quotient of $K^{n}$ by the fat diagonal $K^{n}-K^{(n)}$ ). The $n^{\text {th }}$ homogeneous functor is

$$
\left(D_{n} F\right)(X) \sim\left(\left(K^{(n) c}\right)^{*} \wedge X^{\wedge n}\right)_{h \Sigma_{n}},
$$

and this can be identified with

$$
\operatorname{Map}_{*}\left(K^{(n) c}, \Sigma^{\infty} X^{\wedge n}\right)_{h \Sigma_{n}}
$$

Geometry \& Topology, Volume 7 (2003) 
because $K^{(n) c}$ is finite. It can also be identified with

$$
\operatorname{Map}_{*}\left(K^{(n) c}, \Sigma^{\infty} X^{\wedge n}\right)^{\Sigma_{n}}
$$

because the group action on $K^{(n) c}$ is free.

The analogous conclusion for based $K$ says

$\partial_{y_{1}, \ldots y_{n}}^{(n)} \Sigma_{+}^{\infty}\left(Y, y_{0}\right)^{\left(K, k_{0}\right)} \sim \int_{c}^{k \in\left(K-\left\{k_{0}\right\}\right)^{(n)}} \Sigma_{+}^{\infty}\left(Y, y_{0}, y_{1}, \ldots, y_{n}\right)^{\left(K, k_{0}, k_{1}, \ldots, k_{n}\right)} d k$.

When $Y$ is a point we find that the $n^{\text {th }}$ coefficient of $F(X)=\Sigma_{+}^{\infty}\left(X, x_{0}\right)^{\left(K, k_{0}\right)}$ is the $S$-dual of the one-point compactification of $\left(K-\left\{k_{0}\right\}\right)^{(n)}$.

The case when $K$ is a based circle is particularly simple. Since $\left(S^{1}-\left\{k_{0}\right\}\right)^{(n)}$ is the disjoint union of open $n$-cells freely and transitively permuted by the group, the formula for the $n^{t h}$ homogeneous layer of $\Sigma_{+}^{\infty} \Omega X$ becomes $\Omega^{n} \Sigma^{\infty}\left(X^{\wedge n}\right)$, as already pointed out in 1.20 .

\section{Example: The identity functor}

The identity functor $\mathcal{T} \stackrel{I}{\rightarrow} \mathcal{T}$ from based spaces to based spaces is a central example, and its $n^{\text {th }}$ derivative $\partial^{(n)} I(*)$ is a basic object in homotopy theory. This spectrum with $\Sigma_{n}$-action turns out to be $S$-dual to a certain finite complex with $\Sigma_{n}$-action. We summarize and discuss some known results.

Note that the problem of determining the $n^{\text {th }}$ derivative of the identity is equivalent to that of determining the $n^{\text {th }}$ derivative of the functor $\Omega \Sigma$. In fact, by 5.3 we have

$$
\partial^{(n)} \Omega \Sigma(*) \simeq S^{\bar{V}_{n}} \wedge \partial^{(n)} I(*)
$$

and therefore

$$
\partial^{(n)} I(*) \simeq \Omega^{\bar{V}_{n}} \partial^{(n)} \Omega \Sigma(*)
$$

To begin with the obvious, the first derivative of $I$ is the sphere spectrum.

The second derivative can be identified by using the second cross-effect. The total homotopy fiber of

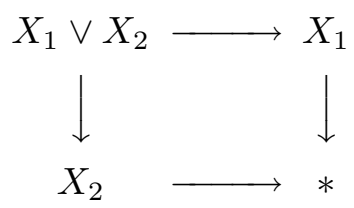

Geometry \& Topology, Volume 7 (2003) 
is the homotopy fiber of $X_{1} \vee X_{2} \rightarrow X_{1} \times X_{2}$, and the bilinearization of this is $\Omega Q\left(X_{1} \wedge X_{2}\right)$, with the obvious $\Sigma_{2}$-symmetry (trivial action on the loop coordinate), simply because there is a map (natural and symmetry-preserving)

$$
\operatorname{hofiber}\left(X_{1} \vee X_{2} \rightarrow X_{1} \times X_{2}\right) \rightarrow \Omega Q\left(X_{1} \wedge X_{2}\right)
$$

that is approximately $3 k$-connected when $X_{1}$ and $X_{2}$ are $k$-connected. It follows that $\partial^{(2)} I(*)$ is the -1 -sphere with trivial action.

For the $n^{\text {th }}$ derivative, partial information can be obtained from the HiltonMilnor theorem [15]. Recall that this describes the space $\Omega \Sigma(X \vee Y)$ as a weak product (direct limit of finite products) of factors each of which has the form $\Omega \Sigma\left(X^{\wedge a} \wedge Y^{\wedge b}\right)$. The factors for a given pair $(a, b)$ correspond to certain nested commutator expressions, an integral basis for the bidegree $(a, b)$ summand of a free Lie ring on two generators whose bidegrees are $(1,0)$ and $(0,1)$. Iteration yields a description of $\Omega \Sigma\left(X_{1} \vee \cdots \vee X_{n}\right)$ as a weak product of factors of the form $\Omega \Sigma\left(X_{1}^{\wedge a_{1}} \wedge \cdots \wedge X_{n}^{\wedge a_{n}}\right)$. The cross-effect $\left(c r_{n} I\right)\left(X_{1}, \ldots, X_{n}\right)$ is the product of those factors for which $a_{j} \geq 1$ for all $j$. The multilinearized cross-effect sees only those factors for which $a_{j}=1$ for all $j$. Thus the $n^{\text {th }}$ differential of the functor $\Omega \Sigma$ is a product of copies of $Q\left(X_{1} \wedge \cdots \wedge X_{n}\right)$, and the $n^{t h}$ derivative is the product of a corresponding number of copies of the sphere spectrum. The number of copies is $(n-1)$ !, and they correspond to a basis for the group Lie $(n)$ generated by all Lie monomials in $n$ variables such that each variable occurs just once (and of course considered modulo the Jacobi identity and antisymmetry). A standard choice of basis for Lie $(n)$ consists of the monomials

$$
\left[x_{\pi(1)},\left[x_{\pi(2)},\left[\ldots\left[x_{\pi(n-1)}, x_{n}\right] \ldots\right]\right]\right]
$$

one for each permutation $\pi$ belonging to the subgroup $\Sigma_{n-1} \subset \Sigma_{n}$. It follows that the $n^{\text {th }}$ derivative of $\Omega \Sigma$, regarded as a spectrum with $\Sigma_{n-1}$-action, is the suspension spectrum of the finite set $\left(\Sigma_{n-1}\right)_{+}$.

Of course for determining $D_{n} I$ one needs the full $\Sigma_{n}$-action. The method outlined above even identifies the action of $\Sigma_{n}$ on the homology of the spectrum $\partial^{(n)} I(*)$ (a free abelian group of rank $(n-1)$ ! concentrated in degree $1-n$ ); it is the obvious action of $\Sigma_{n}$ on $\operatorname{Lie}(n)$, twisted by signs of permutations. But this is still insufficient for determining the homogeneous functor.

Johnson [11] defined a based finite complex $K_{n}$ with $\Sigma_{n}$-action whose $S$-dual is $\partial^{(n)} I(*)$. Her $K_{n}$ was designed to admit an interesting map

$$
\left(c r_{n} I\right)\left(X_{1}, \ldots, X_{n}\right) \rightarrow \operatorname{Map}_{*}\left(K_{n}, X_{1} \wedge \cdots \wedge X_{n}\right),
$$

both natural and symmetry-preserving, and she showed that after multilinearization this map leads to an equivalence

$$
\left(D^{(n)} I\right)\left(X_{1}, \ldots, X_{n}\right) \rightarrow \operatorname{Map}_{*}\left(K_{n}, Q\left(X_{1} \wedge \cdots \wedge X_{n}\right)\right) .
$$


Arone and Mahowald [4] later came up with another answer to the same question. It is defined in terms of the poset of all partitions (equivalence relations) on the set $\underline{n}=\{1, \ldots n\}$. The poset has a maximal element (the trivial partition) and a minimal element (the improper partition), so that the nerve of the poset is (for two reasons) contractible. The Arone-Mahowald version of $K_{n}$ can be described as the double suspension of the nerve of the poset of proper nontrivial partitions, or alternatively as the quotient of the nerve of all partitions by the union of the nerve of the nontrivial partitions and the nerve of the proper partitions.

In [4] this model is justified by proving directly that it is (equivariantly) homotopy equivalent to Johnson's space, but it was actually discovered from a very different point of view, which is worked out in detail in [3]. There is a cosimplicial functor from spaces to spaces which has in degree $d$ the functor $Q^{d+1}$, iterated composition of $Q$ with itself. When applied to 1-connected spaces it serves as a resolution of the identity functor. (In general it gives the Bousfield-Kan integral completion functor.) For each $d$ the functor $Q^{d+1}$ has a split Taylor tower, which can be read off from the Snaith splitting formula. It is rather easy to see that the $n^{\text {th }}$ coefficient of the $d^{\text {th }}$ functor is (functorially in $d$, up to homotopy) the $S$-dual of the (discrete) space of $d$-simplices in $K_{n}$. (The details worked out in [3] dispose of that unfortunate "up to homotopy".)

Arone and Mahowald use this description of $K_{n}$ to investigate the mod $p$ homology of the spectrum $\left(\partial^{(n)} I(*) \wedge X^{\wedge n}\right)_{h \Sigma_{n}}$ whose zeroth space is $\left(D_{n} I\right)(X)$. Their main results concern the case when $X$ is a sphere. For simplicity take it to be an odd sphere. When $n$ is not a power of $p$ they find that the layer $\left(D_{n} I\right)\left(S^{2 m-1}\right)$ is $p$-locally trivial. (This is equivalent to the statement that the homology of $\Sigma_{n}$ with coefficients in $\operatorname{Lie}(n)$ localized at $p$ is trivial.) When $n=p^{k}$ they use Dyer-Lashof operations to calculate the homology as a module over the Steenrod algebra, finding in particular that the layer $\left(D_{p^{k}} I\right)\left(S^{2 m-1}\right)$ has trivial $v_{j}$-periodic homotopy when $j<k$.

For further insight into these matters, see [2].

We close with some remarks about the derivatives of the identity at a general space $Y$. Recall from the discussion following 6.3 that the $n^{\text {th }}$ derivative of $\mathcal{T} \stackrel{I}{\rightarrow} \mathcal{T}$ will be a functor of spaces with $n+1$ basepoints, symmetric with respect to permutations of the last $n$ points. It is clear that

$$
\partial_{y}\left(Y, y_{0}\right) \sim \Sigma_{+}^{\infty} P_{y_{0}}^{y} Y \text {. }
$$

where $P_{y_{0}}^{y} Y$ is the path space $\left(Y, y_{0}, y\right)^{(I, 0,1)}$. In general, the $n^{t h}$ derivative of the identity at an arbitrary space may be described in terms of the $n^{\text {th }}$ 
derivative of the identity at a point. For example, given a space $Y$ and points $y_{0}, y_{1}, y_{2}$, the total homotopy fiber (with respect to $y_{0}$ ) of

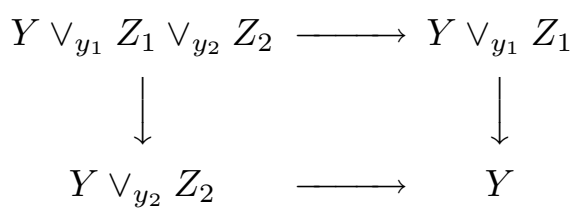

is equivalent to the total homotopy fiber of

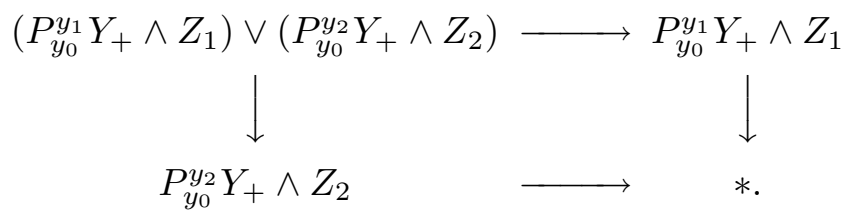

(The second square consists essentially of the homotopy fibers over $y_{0} \in Y$ of the spaces in the first square.) Bilinearizing with respect to $Z_{1}$ and $Z_{2}$, we find that

$$
\left(D_{\left(Y, y_{0}\right)}^{(2)} I\right)\left(Y \vee_{y_{1}} Z_{1}, Y \vee_{y_{2}} Z_{2}\right) \sim\left(D_{*}^{(2)} I\right)\left(P_{y_{0}}^{y_{1}} Y_{+} \wedge Z_{1}, P_{y_{0}}^{y_{2}} Y_{+} \wedge Z_{2}\right) .
$$

The same argument applies for any $n$ and yields a natural and symmetrical equivalence

$$
\partial_{y_{1}, \ldots, y_{n}}^{(n)}\left(Y, y_{0}\right) \sim P_{y_{0}}^{y_{1}} Y_{+} \wedge \cdots \wedge P_{y_{0}}^{y_{n}} Y_{+} \wedge K_{n}^{*}
$$

These observations can be used to give an alternative to the Hilton-Milnor argument above; if one is willing to settle for $\Sigma_{n-1}$-symmetry rather than $\Sigma_{n}$ symmetry, then by 6.3 one can write

$$
\partial_{y_{1}, \ldots, y_{n}}^{(n)}\left(Y, y_{0}\right) \sim \partial_{y_{1}, \ldots, y_{n-1}}^{(n-1)} \Sigma_{+}^{\infty} P_{y_{0}}^{y_{n}} Y,
$$

which by another very slight generalization of 7.10 is equivalent to

$$
\int_{c}^{k \in(I-\{0,1\})^{(n-1)}} \Sigma_{+}^{\infty}\left(Y, y_{0}, y_{1}, \ldots, y_{n-1}, y_{n}\right)^{\left(I, 0, k_{1}, \ldots, k_{n-1}, 1\right)} d k .
$$

In the case $Y=*$ this becomes the $S$-dual of the one-point compactification of $(I-\{0,1\})^{(n-1)}$, in other words the $S$-dual of a wedge of $(n-1)$-spheres freely and transitively permuted by $\Sigma_{n-1}$.

In fact, by taking a different point of view, one can see that the $n^{\text {th }}$ differential or derivative of the identity has a $\Sigma_{n+1}$-symmetry and not just a $\Sigma_{n}$-symmetry, just as if the identity were itself a derivative. We hope to return to this point in a future paper. 


\section{Example: Waldhausen K-theory}

Let $A$ be Waldhausen's algebraic $K$-theory functor from spaces to spectra [15]. In [9] it was shown that

$$
\partial_{y} A(Y) \sim \Sigma_{+}^{\infty} \Omega Y .
$$

We will give similar formulas for the higher derivatives of $A$.

Really what was shown in [9] was

$$
\partial_{y} P^{D i f f}(Y) \sim \Omega^{2} \Sigma^{\infty} \Omega Y,
$$

where $P^{D i f f}$ is stable smooth pseudoisotopy theory. Then 9.1 was a corollary in view of Waldhausen's relation

$$
A(X) \sim W h^{D i f f} X \times \Sigma_{+}^{\infty} X .
$$

where the Whitehead functor $W h^{\text {Diff }}$ satisfies $\Omega^{2} \Omega^{\infty} W h^{D i f f} X \sim P^{D i f f} X$.

A natural map

$$
P^{D i f f} X \rightarrow \Omega^{2} Q\left(X^{S^{1}} / X\right)
$$

played a key role in obtaining 9.2 , where $X^{S^{1}} / X$ is the quotient of the free loopspace $X^{S^{1}}$ by the constant loops. It was then clear that a more direct account of 9.1 ought to involve some analogous map

$$
A(X) \stackrel{\tau}{\rightarrow} L(X),
$$

where $L(X)=\Sigma_{+}^{\infty} X^{S^{1}}$, a map that ought to have a $K$-theoretic rather than a manifold-theoretic description. It is not hard to make such a map (the trace), using the methods of [16].

9.4 Remark This was generalized by Bokstedt. The trace map $\tau$ is reminiscent of the Dennis trace map from the algebraic $K$-theory of a ring to its Hochschild homology, and this observation pointed the way to the generalization. Since $A(B G)$ can be defined as the algebraic $K$-theory of a generalized ring which may be thought of as the group ring of $G$ over the sphere spectrum, and since the Hochschild homology of a group ring $k[G]$ is the homology of $(B G)^{S^{1}}$ with coefficients in $k$, it was natural to imagine that $\tau$ should be a special case of a construction

$$
K(R) \rightarrow T H H(R)
$$

defined for reasonable ring spectra $R$, where $T H H(R)$ is a kind of "Hochschild homology over the sphere spectrum". Bokstedt invented (and named) the object THH and defined the trace (originally for a class of generalized rings called functors with smash product ). See [6]. 
The trace induces a map

$$
\partial_{y} A(Y) \stackrel{\partial_{y} \tau}{\longrightarrow} \partial_{y} L(Y)
$$

By 7.1 we have

$$
\partial_{y} L(Y) \sim \int^{k \in S^{1}} \Sigma_{+}^{\infty}(Y, y)^{\left(S^{1}, k\right)} d k .
$$

Using rotations of the circle to continuously identify $\left(S^{1}, k\right)$ with $\left(S^{1}, 1\right)$, where 1 is one point in $S^{1}$, this last spectrum may be identified with

$$
\int^{k \in S^{1}} \Sigma_{+}^{\infty}(Y, y)^{\left(S^{1}, 1\right)} d k \cong \operatorname{Map}_{*}\left(S_{+}^{1}, \Sigma_{+}^{\infty} \Omega_{y} Y\right),
$$

and thus split into two factors $\Sigma_{+}^{\infty} \Omega_{y} Y \times \Omega \Sigma_{+}^{\infty} \Omega_{y} Y$. Projecting on the first factor and composing with $\partial_{y} \tau$ we get a map

$$
\partial_{y} A(Y) \rightarrow \Sigma_{+}^{\infty} \Omega_{y} Y
$$

which is in fact an equivalence.

9.5 Remark It would have been very tedious to prove this last fact directly from 9.2. That would have meant combining the proofs of 9.2 and 9.3 and the construction of the trace and undoubtedly dealing with several different models for $A(X)$. Instead in [5] we took a shortcut, observing that the functors $\partial_{y} A(Y)$ and $\Sigma_{+}^{\infty} \Omega_{y} Y$ are abstractly equivalent by 9.1 , and then arguing by universal examples that a natural map between them must be an equivalence for all $Y$ if this is so in some special cases where things can be checked.

It is possible, and very convenient, to use a homotopy fixed point construction to single out the factor of $\partial_{y} L(Y)$ that is to correspond to $\partial_{y} A(Y)$. Consider the obvious action of the circle group $T$ on $L$. The trace can easily be made to factor through the homotopy fixed point spectrum

$$
A(Y) \stackrel{\tilde{\tau}}{\rightarrow} L(Y)^{h T} \rightarrow L(Y),
$$

and it was shown in [5] that the map

$$
\partial_{y} A(Y) \rightarrow\left(\partial_{y} L(Y)\right)^{h T}
$$

resulting from this refined trace $\tilde{\tau}$ is an equivalence. This form of 9.1 will be very useful for getting the higher derivatives.

We emphasize that the right hand side of 9.6 is not $\partial_{y}\left(\left(L(Y)^{h T}\right)\right.$, and that $L^{h T}$ is not an analytic functor. There is a canonical map

$$
\partial_{y}\left(\left(L(Y)^{h T}\right) \rightarrow\left(\partial_{y} L(Y)\right)^{h T},\right.
$$

because $T$ acts continuously on the homotopy functor $L$; but this map does not happen to be an equivalence. 
9.7 Theorem For all $n \geq 1$ the (natural and symmetry-preserving) map

$$
\partial_{y_{1}, \ldots, y_{n}}^{(n)} A(Y) \rightarrow\left(\partial_{y_{1}, \ldots, y_{n}}^{(n)} L(Y)\right)^{h T}
$$

induced by $\tilde{\tau}$ is an equivalence.

Proof In proving this we are allowed to ignore the symmetry; thus for this purpose we can get away with using 6.3 to view $n^{\text {th }}$ derivatives as first derivatives of $(n-1)^{s t}$ derivatives.

The key point now is that the operations $\partial_{y}$ and ()$^{h T}$, which did not commute when applied to $L(Y)$, do commute when applied to $\partial_{y_{2}, \ldots, y_{n}}^{(n-1)} L(Y)$. Once this is established we can argue by induction on $n$ :

$$
\partial_{y_{1}} \partial_{y_{2}, \ldots, y_{n}}^{(n-1)} A(Y) \sim \partial_{y_{1}}\left(\left(\partial_{y_{2}, \ldots, y_{n}}^{(n-1)} L(Y)\right)^{h T}\right) \sim\left(\partial_{y_{1}} \partial_{y_{2}, \ldots, y_{n}}^{(n-1)} L(Y)\right)^{h T} .
$$

What is needed to establish it is the equation

$$
\partial_{y_{2}, \ldots, y_{n}}^{(n-1)} L(Y) \sim \int_{c}^{k \in\left(S^{1}\right)^{(n-1)}} \Sigma_{+}^{\infty}\left(Y, y_{2}, \ldots, y_{n}\right)^{\left(S^{1}, k_{2}, \ldots, k_{n}\right)} d k
$$

(an instance of 7.10), together with the observation that $T$ is acting freely on $\left(S^{1}\right)^{(n-1)}$

Now apply the principle given at the end of Section 5 , taking $G$ to be $T, P$ to be $\left(S^{1}\right)^{(n-1)}$ and $B$ to be the orbit space. It is clear from 9.6 that for a suitable system $E$, functorial in $\left(Y, y_{2}, \ldots, y_{n}\right)$, we have

$$
\partial_{y_{2}, \ldots, y_{n}}^{(n-1)} L(Y) \sim \int^{p \in P} E_{\pi(p)} d p
$$

equivariantly and

$$
\begin{aligned}
\partial_{y}\left(\left(\int^{p \in P} E_{\pi(p)} d p\right)^{h T}\right) & \sim \partial_{y} \int^{b \in B} E_{b} d b \\
& \sim \int^{b \in B} \partial_{y} E_{b} d b \\
& \sim\left(\int^{p \in P} \partial_{y} E_{\pi(p)} d p\right)^{h T} \\
& \sim\left(\partial_{y} \int^{p \in P} E_{\pi(p)} d p\right)^{h T} .
\end{aligned}
$$

The differentiations under the integral are justified by the finiteness of $B$ and of $P$. (This step fails when $n=1$, basically because the appropriate $B$ would then be the space $B T$, which is not so finite.) 
In the special case $Y=*$ the conclusion of 9.7 is that the $n^{t h}$ derivative of $A$ is the homotopy fixed point spectrum for $T$ acting on the $S$-dual of the onepoint compactification of $\left(S^{1}\right)^{(n)}$, where $\left(S^{1}\right)^{(n)}$ has the obvious commuting actions of $T$ and $\Sigma_{n}$. Since the $T$-action is free, the answer may be rewritten as the $S$-dual of the one-point compactification of $\left(S^{1}\right)^{(n)} / T$. As a $\Sigma_{n}$-space, $\left(S^{1}\right)^{(n)} / T$ is isomorphic to $\Sigma_{n} \times C_{n}\left(S^{1} \times \bar{V}_{C_{n}}\right)$, where the cyclic group $C_{n}$ acts on $S^{1}$ as a subgroup of the rotations and acts linearly on the vector space $\bar{V}_{C_{n}}$ by the reduced regular representation. It follows that the $n^{\text {th }}$ derivative of $A$ at a one-point space is induced from $C_{n}$ and the $n^{\text {th }}$ layer of the Taylor tower is given by

$$
\left(D_{n} A\right)(X) \sim \operatorname{Map}_{*}\left(S^{1}+\wedge S^{\bar{V}_{C_{n}}}, \Sigma^{\infty}\left(X^{\wedge n}\right)\right)^{C_{n}} .
$$

9.9 Remark In the special case when $X$ is a suspension $\Sigma Y$ this becomes $\Sigma \operatorname{Map}_{*}\left(S^{1}+, \Sigma^{\infty}\left(X^{\wedge n}\right)\right)^{C_{n}}$. In fact, in that case the Taylor tower splits:

$$
A(\Sigma Y) \sim A(*) \times \prod_{n \geq 1} \Sigma M a p_{*}\left(S^{1}+, \Sigma^{\infty}\left(X^{\wedge n}\right)\right)^{C_{n}}
$$

if $Y$ is connected. A proof of this was sketched in [7] and corrected in [5]. This special case of the conclusion was used in the shortcut mentioned in 9.5.

9.10 Remark There is also a direct $K$-theoretic approach to all of this. Dundas and McCarthy [8] generalized 9.1 to (generalized) rings. Their statement is that the trace map induces an equivalence from "stable $K$-theory" to $T H H$. This led in [13] to an extension of the main result of [5] to such generalized rings.

\section{References}

[1] G Arone, A generalization of Snaith-type splitting, Trans. AMS 351 (1999) $1123-1150$

[2] G Z Arone, W G Dwyer, Partition complexes, Tits buildings, and symmetric products, Proc. London Math. Soc. 3 (2001) 229-256

[3] G Z Arone, M Kankaanrinta, A functorial model for iterated Snaith splitting with applications to calculus of functors, from: "Stable and unstable homotopy", AMS 1-30 (1996)

[4] G Arone, M Mahowald, The Goodwillie tower of the identity functor and the unstable periodic homotopy of spheres, Invent. Math. 135 (1999) 743-788

[5] M Bokstedt, G Carlsson, R Cohen, T Goodwillie, W-C Hsiang, I Madsen, On the Algebraic K-Theory of Simply Connected Spaces, Duke Math. J. 84 (1996) 541-563 
[6] M Bokstedt, W-C Hsiang, I Madsen, The cyclotomic trace and algebraic K-theory of spaces, Invent. Math. (1993) 463-539

[7] G Carlsson, R Cohen, T Goodwillie, W-C Hsiang, The free loopspace and the algebraic $K$-theory of spaces, $K$-theory 1 (1987) 53-82

[8] B Dundas, R McCarthy, Stable $K$-theory and topological Hochschild homology, Annals of Math. 140 (1994) 685-702

[9] T Goodwillie, Calculus I: The First Derivative of Pseudoisotopy Theory, KTheory 4 (1990) 1-27

[10] T Goodwillie, Calculus II: Analytic Functors, K-Theory 5 (1992) 295-332

[11] B Johnson, The derivatives of homotopy theory, Trans. AMS 347 (1995) 12951321

[12] L G Lewis, J P May, M Steinberger, Equivariant Stable Homotopy Theory, Lecture Notes in Mathematics 1213, Springer-Verlag (1986)

[13] R McCarthy, Relative algebraic $K$-theory and topological cyclic homology, Acta Math. 179 (1997) 197-222

[14] S Mac Lane, Categories for the Working Mathematician, Springer-Verlag (1971)

[15] F Waldhausen, Algebraic $K$-theory of spaces, Lecture Notes in Mathematics 1126, Springer-Verlag (1985) 318-419

[16] F Waldhausen, Algebraic $K$-theory of topological spaces, II, Lecture Notes in Mathematics 763, Springer-Verlag (1979) 356-394

[17] G Whitehead, Elements of Homotopy Theory, Springer-Verlag (1978) 